\title{
One Lab, Two Firms, Many Possibilities: on R\&D outsourcing in the biopharmaceutical industry*
}

\author{
Etienne Billette de Villemeur ${ }^{\dagger}$ Bruno Versaevel ${ }^{\ddagger}$
}

This version: January 2019

\begin{abstract}
We draw from documented characteristics of the biopharmaceutical industry to construct a model where two firms can choose to outsource R\&D to an external unit, and/or engage in internal R\&D, before competing in a final market. We investigate the distribution of profits among market participants, and the incentives to coordinate outsourcing activities or to integrate $R \& D$ and production. Consistent with the empirical evidence, we find that the sign and magnitude of an aggregate measure of direct (inter-firm) and indirect (through the external unit) technological externalities drives the distribution of industry profits, with higher returns to the external unit when involved in development (clinical trials) than in early-stage research (drug discovery). In the latter case, the delinkage of investment incentives from industry value, together with the ability of firms to transfer risks to the external unit, imply a vulnerability of early-stage investors' returns to negative shocks, and the likely abandonment of projects with economic and medical value. We also find that competition in the equity market makes a buyout by one of the two firms more profitable to a research biotech than to a clinical services unit, and can stimulate early-stage investments. However, this long-term incentive can be minimal, notably if the superior efficiency of outsourced operations originates from economies of scope that can hardly be exploited when a firm takes control of the external unit exclusively for itself. R\&D outsourcing thus does not always qualify as a relevant pathway to address the declining productivity in innovation that has characterized the industry over several decades.
\end{abstract}

JEL classification: L13; L65; O31.

Keywords: research; development; biotechnology; pharmaceuticals; externalities.

*A previous version of this paper was circulated under the title "Conflict and Cooperation on R\&D markets." The authors are grateful to Martin Chalkley (Editor), David Bardey, Izabela Jelovac, Didier Laussel, Lise Rochaix, Richard Ruble, Désiré Vencatachellum, an anonymous referee, and seminar participants at Hospinnomics (PSE \& AP-HP), LEM/Universite de Lille, and the 19th European Health Economics Workshop, for comments and discussions. All remaining errors are ours.

†Université de Lille \& LEM UMR 9221, France.

‡EM Lyon Business School \& GATE L-SE UMR 5824, France. Corresponding author (versaevel@em-lyon.com). 


\section{Introduction}

A decline in R\&D (research and development) productivity has been observed in the biopharmaceutical industry over several decades (Munos, 2009; Pammolli et al., 2011). While the number of new molecular entities and biologics that are approved annually by the US Food and Drug Administration (FDA) has remained around the same level since 1950, when measured per billion US dollars spent on R\&D this number has halved roughly every 9 years (Scannell et al., 2012). The scale of the productivity problem can be gauged by considering changes in the average full cost estimate of bringing a new compound to the market. This estimate is $\$ 451$ million in DiMasi et al. (1991), \$1,031 million in DiMasi et al. (2003), and \$2,558 million in DiMasi et al. (2016). ${ }^{1}$

There is also evidence that pharmaceutical companies that engage in internal R\&D increasingly outsource specific tasks: "The global drug discovery outsourcing market was USD 14.9 billion (2014) and is expected to reach USD 25 billion by 2018, while the market for CRO [contract research organization]-conducted clinical trials was USD 23.1 billion (2014) and is expected to increase to USD 35.8 billion by 2020 "(Schuhmacher et al., 2016, p. 8). It is believed in the industry that R\&D outsourcing can reduce costs by increasing efficiency in the discovery and testing steps toward new medicines (e.g., Taylor et al., 2016; Sancheti et al., 2018). On the demand side of the R\&D market, the tasks that pharmaceutical companies choose to contract out cover a large range of activities, including target validation, genetic engineering, chemical synthesis, compound screening, lead compounds evaluation, preclinical studies, and clinical (in-human) trials. On the supply side, science-based biotech firms specialize in early-stage research activities for the discovery of new chemical or biological molecules, while CROs deliver later-stage development services for the safety and efficacy assessment of drug or vaccine candidates.

Despite the observed decline in R\&D productivity, as gross margins in medication markets have evolved in parallel with R\&D spending, the net profit returns in the biopharmaceutical industry have remained persistently high at the aggregated level since the early 1960s (Scherer, 2001, 2010). However, the average profitability of biotech units that specialize in the discovery of new treatments

\footnotetext{
${ }^{1}$ Here we refer to studies based on the same methodology to estimate costs, which include out-of-pocket R\&D costs and time cost (i.e., cost of capital). The estimates in DiMasi et al. (1991, 2003) have been updated to US\$ 2011 prices in Mestre-Ferrandiz et al. (2012), and the one in DiMasi et al. (2016) is in 2013 prices. The three estimates are based on compound-level data, with initial human testing that occurred during the periods 1970-1982, 1983-1994, and 1995-2007, respectively.
} 
or preventives has remained low since the emergence of genetic engineering in the 1970s (Pisano, 2006a, 2010). Biotech firms also face greater financial risk and are more sensitive to policy shocks that affect expected future profitability than the average large pharmaceutical company (Golec and Vernon, 2009). A recent empirical analysis of a comprehensive sample of 1,066 companies in the period 1930-2015 confirms these observations by disaggregating the financial performance across the pharmaceutical and biotech subsectors: "[o]ur empirical results show that investments in the pharmaceutical industry have outperformed the broader stock market over a long period of time, whereas investments in the biotech industry have underperformed the market" (Thakor et al., 2017, p. 1154), and the performance differential appears especially pronounced after 2001. Moreover, by decomposing the risk of the pharma and biotech portfolios into factors arising from general economic conditions (systematic risk) and factors that are unique to the individual company or project (idiosyncratic risk), it is found that the majority of the total risk for the pharmaceutical subsector is of the systematic type while, in contrast, "the biotech portfolio has both systematic risk and idiosyncratic risk, with a much higher idiosyncratic risk than in the pharma portfolio" (p. 1153). Moreover, the systematic risk in biotech firms is as large as - and in some periods can even be substantially higher than that of the pharma companies. The latter empirical analysis does not include the subsector of CROs involved in the clinical assessment of drug or vaccine candidates. For these organizations, business information abounds that describes more favourable financial conditions, such as "high visibility of revenues, excess cash generation, strong balance sheets and limited exposure to a number of risks that commonly affect biopharma companies ..." (Bali et al., 2013, p. 3). As a consequence, unlike biotech firms, "CROs have historically traded at a 21\% premium to the S\&P 500 over the past 14 years" (Wilson et al., 2016, p. 41).

How does the distribution of industry profits among pharma companies, biotech firms, and CROs, relate to the functioning of the R\&D market? Can technological characteristics of contractedout operations explain the low average profitability of biotech units, and the higher financial returns of CROs? Are the risks inherent to discovery and clinical development activities mitigated by outsourcing contracts? What are the consequences for early-stage investment incentives? In order to answer these questions, we draw on documented characteristics of the biopharmaceutical industry to construct a model in which a for-profit upstream external unit (e.g., a biotech startup, or a contract research organization) conducts specific R\&D tasks as solicited non-cooperatively by two downstream firms (big pharma), which also run R\&D operations internally before competing in a final market. 
The external unit interacts with the two firms by responding to their contract offers, and can choose to serve both firms, only one, or none. The firms can substitute internal resources for some or all of the external unit's operations, and their contract offers reflect multi-stage strategic interactions in the intermediate R\&D market and in the final product market. Overall, the formal analysis leads to new insights on the functioning of R\&D outsourcing and its implications for biopharmaceutical firms and their external partners, with strong and intelligible connections to the recent empirical evidence on the relation between contracted-out and in-house technological activities, on the nature of knowledge externalities in discovery and (distinctively) clinical development, and on merger activity in the equity market.

More specifically, our main results establish simple conditions on the model primitives for the total equilibrium R\&D benefits to be either fully appropriated by the two firms, or partially retained by the external unit. These conditions, which can explain the persistently low average profitability of biotech firms, relate to indirect and direct technological externalities. There are indirect technological externalities if the cost of R\&D, as conducted by the external unit for the two firms, reflects economies or diseconomies of scope. There are direct technological externalities if some of the knowledge received or produced by a firm impacts the gross profit of its competitor. We connect the sign and magnitude of these externalities with the distribution of profits among participants in the intermediate market for R\&D.

Situations of positive indirect (through the external unit) and direct (inter-firm) externalities correspond mainly to early-stage discovery activities, in which biotech units are typically involved, and where economies of scope across research projects and significant inter-firm knowledge spillovers have been evidenced in the empirical literature (Henderson and Cockburn, 1996). ${ }^{2}$ In the theoretical context of our model, we find that the external unit exactly breaks even, and hence investors have no positive incentive to engage financial resources, precisely in circumstances where outsourcing the tasks of the most fundamental nature can reduce the costs of discovering new medicines. We also demonstrate that downstream firms can use risk-proof contract offers in order to transfer - at least partly - the burden of unfavorable realizations of uncertainty to the external unit, via adjusted payments. With an expected payoff at the upstream stage equal to zero, an unfavorable realization of uncertainty then implies a negative return, and thus possibly leads the external unit to shut down,

\footnotetext{
${ }^{2}$ In a survey, Hernandez-Villafuerte et al. (2017) observe that, although the evidence is mixed, in biomedical and health research the literature more often points to the existence of positive economies of scope than diseconomies.
} 
although its activities generate a positive - and possibly very high - industry profit. This (intermediate) market failure outcome suggests that protection measures for the external unit (e.g., a lower bound to milestone payments in case of adverse events) can be necessary to avoid the abandonment of socially valuable research projects.

However, in our model, when an aggregate measure of technological externalities is negative, the external unit can appropriate a positive share of total profits that can be derived analytically. In that case the downstream firms earn only their marginal contribution to the industry value, and the payoff to the external unit depends on the magnitude of externalities. This situation is consistent with the empirical studies that identify diseconomies of scope and nonexistent spillovers in the late-phase clinical trials of candidate drugs (Danzon et al. 2005; Macher and Boerner, 2006), such as conducted by specialized CROs. Then the positive profit to the external unit is an effect of competition between the contract offers of the two firms for the orientation of R\&D resources toward specific needs. The intensity of that competition depends on the nature of R\&D activities - as conducted by the external unit and by the two firms - and then on the effect of these activities on downstream cost and demand characteristics, which in the end also impact final market interactions.

We also connect the technological conditions that drive the distribution of $R \& D$ benefits to the firms' incentives to participate in the equity market, where big pharma companies acquire specialized innovative units involved in promising research or clinical development programs. For example, in the oncology domain, AbbVie agreed to buy Pharmacyclics for $\$ 21$ billion in 2015, and Pfizer acquired Medivation for $\$ 14$ billion in 2016. Such acquisitions, which substitute for contractual outsourcing relations, are viewed by industry leaders as another response to the declining R\&D productivity problem (Comanor and Scherer, 2013). Still the high transaction prices observed in the equity market suggest that pharmaceutical firms pay more for R\&D by acquiring an external unit than by contracting with it as a partner, or by carrying out the R\&D internally (Pisano, 2015). Acquisitions usually conclude a bidding contest where several big pharma rivals compete for the same buyout target, ${ }^{3}$ leading to equity valuations often considered excessive by industry analysts and experts. ${ }^{4}$

\footnotetext{
${ }^{3}$ For example, "[f]or several days, Johnson \& Johnson was considered the most likely acquirer of Pharmacyclics ... But AbbVie stepped in with a higher bid ..." (www.nytimes.com, March 5, 2015). In the same vein, "AstraZeneca Plc and Pfizer Inc. are among firms considering a counteroffer for Medivation Inc., challenging Sanofi's \$9.3 billion bid for the company ..." (www.bloomberg.com, April 29, 2016).

${ }^{4}$ To illustrate, "AbbVie shares were down 3\% in Thursday trading, as some investors and analysts expressed concern the company was overpaying for Pharmacyclics ..." (www.wsj.com, May 5, 2015); and "GlaxoSmithKline CEO Andrew Witty questioned the ... valuations of recent deals" ... and stated that "[s]ome of these valuations look stretched." (www.firstwordpharma.com, May 11, 2015).
} 
Two categories of cases are identified that depend on the ability of firms to bid or not for the external unit, in relation to financial, managerial, or governance constraints of all kinds. If such constraints are binding, either positive technological externalities dominate and the firms remain independent, or negative externalities lead the firms to choose to coordinate horizontally their R\&D outsourcing (as in Majewski, 2004). Otherwise, should the firms be unable to commit to not unilaterally considering vertical integration, one of them does acquire the external unit. Whether one or the other firm is the acquirer occurs with equiprobability, independently of firm asymmetries. The main outcome is that the competition for the control of the external unit leads to overbidding (an issue discussed in Higgins and Rodriguez, 2006), although the premium can be nil in the case of negative externalities (diseconomies of scope and inexistant inter-firm spillovers), as characteristic of late-stage development tasks. We thus obtain that principally biotech founders engaged in early-stage research - unlike owners of contract research organizations - may reappropriate in the equity market part of the value transferred to their sponsors in the R\&D market.

Our formal analysis relates to a stream of theoretical papers - starting with Aghion and Tirole (1994), Anton and Yao (1994), Bhattacharya and Guriev (2006, 2013) - which investigate a variety of issues that pertain to R\&D outsourcing, although most models in that stream of literature ${ }^{5}$ adopt a general approach with no specific reference to the biopharmaceutical domain. Our approach is complementary to recent analyses by Spulber (2013) and Allain et al. (2015) that characterize the connection between the intensity of downstream competition - which depends on the number of client firms - and upstream incentives to engage in R\&D operations. As in Spulber (2013), in our model an appropriability problem may lead to integrate R\&D and production vertically, and as in Allain et al. (2015) the vertical division of labor in R\&D operations is driven by the nature of competition among client firms. A distinctive feature of our model is that the intensity of competition is a consequence of the exact effects of external and internal R\&D operations on cost and demand conditions. We also share with Ho (2009) and Lai et al. (2009) the assumption that the downstream firms write contract offers, to which the external unit responds, and which can lead to multi-contracting. Moreover, in our model the firms condition their payments on a measure of the external unit's operations, as in Vencatachellum and Versaevel (2009). It follows that the external unit's operations are not conducted before the client firms' choice of payment schemes, consistently with contractual situations in the biopharmaceutical context. These payment schemes are the firms' instruments that determine

\footnotetext{
${ }^{5}$ We review that literature in Billette de Villemeur and Versaevel (2017).
} 
the equilibrium distribution of industry profit among contracting parties.

As the firms' non-cooperative choices of payment schemes depend on technological characteristics of R\&D activities, our model specifications also relate to the empirical evidence on the existence of economies of scale or scope in early-stage research and later-stage clinical development. By using data collected at the firm level and at the level of individual R\&D projects, Henderson and Cockburn (1996) find economies of scope and significant knowledge spillovers in early-stage drug "discovery" tasks (those on with biotech units typically focus). Conversely, Danzon et al. (2005) and Macher and Boerner (2006) identify diseconomies of scope and nonexistent spillovers in the late-phase clinical trials of candidate medicines (the "development" activities that are usually outsourced to specialized CROs). Our main theoretical propositions clearly echo these contrasted empirical results, and show their relevance for explaining the distribution of industry profits between an external unit and its client firms.

The paper is organized as follows. Characteristics of the biopharmaceutical industry are presented in Section 2, in connection to the model specifications introduced in Section 3. The distribution of industry profits is characterized in Section 4, and incentives to shift to a more integrated structure are investigated in Section 5. Final remarks are in Section 6. All proofs are relegated to the Appendix.

\section{The Industry Context}

We derive our theoretical results from model specifications that are carefully related to documented characteristics of the biopharmaceutical "market for technology" (Arora et al., 2001, 2004a), where the industry usually divides research and development activities into two sets. The early-stage research (" $\left.\mathrm{R}^{\prime \prime}\right)$ consists of the discovery of new chemical compounds, vaccine candidates, or other biologics. The later-stage development (" $\mathrm{D}$ ") tasks aim at assessing the safety and efficacy of the therapeutic or prophylactic properties of a candidate medicine on increasingly large populations of individuals.

Outsourced R\&D activities include early-stage research in the biotechnology field, where from the early years onward, "[b]ecause different commercial products were based on similar basic technologies, the costs ... could be shared by clients" (Pisano, 1991, p. 241) and then "[v]irtually every new entrant ... formed at least one, and usually several, contractual relationships with established 
pharmaceutical ... companies" (Pisano, 2006a, p. 87). ${ }^{6}$ A historical example involves the pioneering biotech firm Genentech, which started collaborations with Ely Lilly in 1978 for the synthesis of human insulin, and with Hoffman-LaRoche the same year for the synthesis of interferons. A more recent example involves CureVac, which contracted with Crucell (a Johnson \& Johnson company) in 2013, and with Sanofi Pasteur in 2014, for the funding of R\&D operations toward prophylactic vaccines. Another recent example involves Moderna Therapeutics, which partnered with AstraZeneca in 2016, and with Merck a few months later, for the co-discovery and development of RNA-based candidates for the treatment or prevention of a range of cancers. In our model, accordingly, an external unit can serve up to two client firms simultaneously. ${ }^{7}$

Another important characteristic of the current market for biotechnology is that established pharmaceutical firms do not only give a biotech firm (external unit) access to finance and to manufacturing or marketing resources, they also operate internal biotechnology functions. The situation was different when the biotechnology market emerged in the late 1970s. At that time there was a clear dichotomy in the R\&D focus of suppliers and buyers. On the supply side, a typical new biotech firm used advances in biological sciences - e.g., recombinant DNA technology - for the design of a therapeutic agent. On the demand side, the established pharmaceutical firms, whose technological competence focused on the random screening of compounds against disease targets, procured research in the market for biotechnology before engaging in clinical development. Since then, the largest pharmaceutical firms have acquired capabilities in cell and molecular biology (Galambos and Sturchio, 1998; Rydzewski, 2008), so that the dichotomy has eroded: "[e]stablished firms have embraced biological approaches, including genomics, to drug discovery, while 'biotech firms' employ chemistry" (Pisano, 2006a; p. 17).

Outsourced R\&D also relates to late-stage development activities. Once a new compound, or a candidate vaccine, has been discovered, and tested in animal models, it must go through clinical trials conducted on human subjects. These trials need to produce evidence of safety and efficacy, as required for regulatory approval by government agencies (e.g., the FDA) before market introduction. There are three phases that involve increasingly large samples of subjects (from a few dozen in phase

\footnotetext{
${ }^{6}$ According to Higgins (2007), who uses a large data set of alliances in the biopharmaceutical industry, from 1994 to 2001 each biotechnology firms had on average six alliance partnerships with large pharmaceutical firms.

${ }^{7}$ Although the biotech and CRO industries are highly fragmented (see Argyres and Liebeskind, 2002, and Getz, 2007, respectively), the specification that only one external unit faces two client firms is consistent with the observation that several big pharma companies usually compete for a differentiated technology or expertise as specifically supplied by a given entity among several others (a case of monopolistic competition).
} 
1 to, in case of success, several hundred in phase 2, and then to several thousand subjects in phase 3). As in the case of biotechs, a CRO that supplies clinical trial services can enter in simultaneous contractual relationships with several clients. For example, Parexel entered in 2011 into multi-year contractual agreements with both Merck and Pfizer. The contracting firms do not restrict their strategies to either make or buy clinical trial services as "[f]or a given study, sponsors can choose to retain some functions in house while contracting out others" (Azoulay, 2004; p. 1594). By outsourcing the latter tasks the firms attempt to benefit from economies of scale and scope (Macher and Boerner, 2006), and thereby to reduce their clinical trial costs, which are estimated at around US\$220 million for a new drug (Mestre-Ferrandiz, Sussex, and Towse, 2012). ${ }^{8}$

In our model, the respective efforts of the external unit and its client firms are endogenous, so that the vertical division of R\&D activities can occur at any point between total outsourcing and full integration. This specification is consistent with the observation that the collaboration of big pharma firms with biotech units or clinical trial providers creates joint inputs across the two sides of the contractual relationship, with an exact balance that might vary significantly on a case by case basis.

The large pharmaceutical firms on the demand side of the intermediate market for technology are likely to design the contracts that organize the relationship with an external supplier of discovery activities or of clinical trial services. This is explained by the fact that, when internal resources are available, the capacity of established pharmaceutical firms to "go for it alone" - though possibly at a higher cost - increases their bargaining power (Arora et al., 2004b). Other factors include the severe financial constraints faced by specialized biotech units (Lerner and Merges, 1998; Golec and Vernon, 2009), together with a high rate of entry on the fragmented supply side (Rothaermel, 2001; Argyres and Liebeskind, 2002) while incumbents on the demand side remain highly concentrated. Although the latter structural features describe a "buyer's market", we show that they cannot fully explain the persistently low average profitability of biotech firms since the late 1970s (Pisano, 2006a, 2010). Indeed, in the analysis that follows we identify circumstances where the external unit appropriates the total industry profit, for any probability of success, with client firms that behave as principals and are no less informed than the common independent contractor.

R\&D contracts can incorporate complex clauses to fine tune the financial mechanism (equity par-

\footnotetext{
${ }^{8}$ The out-of-pocket cost of clinical testing depends on the number of patients required to collect sufficient data as demanded from regulatory agencies. It is even higher in the case of preventive vaccine candidates, as the size of human subject test samples is often larger than for drugs (Scherer, 2011; Keith et al., 2013).
} 
ticipation, milestone payments, licensing fees, royalties, ...) $)^{9}$ with the technology or services supplied (or not) by the external unit to other client firms. Non-compete clauses delineate the know-how or expertise that the external unit may or may not use with or provide to a third party, except as expressly notified. ${ }^{10}$ For example, in an early-stage agreement between the pharma company Glaxo and the Canadian research unit BioChem, the latter "shall have the right to contract with third parties for the performance of work, or the provision of consulting services, in connection with the sponsored research program, provided that Glaxo shall have the opportunity to review, comment on and approve any such proposed contract prior to its execution"(p. 9). ${ }^{11}$ Other clauses modulate the payments received by the external unit from the pharma sponsor in connection to the technology that can be supplied to a competitor. To illustrate, a research, development, and license agreement between the pharma company Bristol-Myers Squibb (BMS) and the biotech Ligand Pharmaceuticals (Ligand) stipulates that "[i]n the event that ... Ligand or a Ligand Affiliate, or a Third Party with whom Ligand had collaborated with respect to the development of a Competing Product ... sell such Competing Product in any country in the Territory in which BMS ... is then marketing a Product, ... then ... all royalty rates payable by BMS in each such country shall be reduced by ${ }^{* * *}$ during the period that both such products are marketed in such country"(pp. 27-28). ${ }^{12}$ Accordingly, in our model each firm can condition its payment on the verifiable operations conducted inside the external unit, including those that relate to a third party. ${ }^{13}$ This assumption does not mean that a technology received from the external unit cannot partly benefit a competitor. Unsolicited and non-contractible knowledge spillovers, both through the external unit and across firms, are introduced in the analysis. ${ }^{14}$

Circumstances have been studied in the economics literature where a research unit can (re)sell se-

\footnotetext{
${ }^{9}$ In practice, biotech firms typically receive an upfront fee, milestone payments conditioned on the occurrence of a predetermined event, and sales-based royalties if their intellectual property is in-licensed by the downstream partner, while CROs usually receive a negotiated fee for development services.

${ }^{10}$ Non-compete clauses typically include a "right of first refusal" (Folta, 1998; Hagedoorn and Hesen, 2007) that allows a firm to purchase the rights - or only a selection - to R\&D outcomes before such an option is offered to other firms.

${ }^{11}$ The features of this contractual agreement, dated Jan. 1990, are discussed in Robinson and Stuart (2000).

${ }^{12}$ The contract, dated January 1st 1990, is available at: http://contracts.onecle.com/alpha, where the signs ${ }^{* * *}$ refer to undisclosed confidential information.

${ }^{13}$ The common agency model in Section 3 is thus of the public kind (Martimort, 2007). This specification, in line with the stylized facts presented in section 2, differs from most papers that focus on consumer goods markets where less sophisticated non-compete clauses, hardly verifiable activities, and various antitrust regulations can justify the assumption that a principal can contract exclusively on what it specifically receives from the agent, with no possible connection between payments and the other activities of the agent that benefit a competitor (e.g., Bernheim and Whinston, 1998).

${ }^{14}$ In Cohen et al. (2000) survey data show that firms in the pharmaceutical domain rely more on patents as a protection mechanism than in any other industries. Patenting is an important source of technological spillovers, as it "involves a leakage of a certain portion of the knowledge to the public in the process of filing a patent application"(Bhattacharya and Guriev, 2006, p. 1114).
} 
cretly its technology, and is more knowledgeable than its downstream clients. ${ }^{15}$ In the biopharmaceutical context, secret reselling is unlikely when biotech firms and clinical trial suppliers alike enhance their reputation by communicating on their contractual partners and on the content of agreements. ${ }^{16}$ It can also be the case that pharma firms with internal R\&D operations are more informed on the technological potential of a research program than an external unit engaged in the "least intimate" form of fee-for-service agreement (Pisano, 2006a, p. 108), for example to perform systematic tests on a compounds library, or than a CRO involved in the most "routinized part" (Shuchman, 2007, p. 1367) of clinical trials services. So in our theoretical framework the two firms are not less competent than the external unit, and are not threatened by some form of misbehavior. Our model specifications are rather motivated by the observation that, in science-based businesses, "[p]rofound and persistent uncertainty, rooted in the limited knowledge of human biological systems and processes, makes drug R\&D highly risky" (Pisano, 2006b; p. 119) to all undertakings. We thus focus on situations of "symmetric uncertainties" (Arora and Gambardella, 2010, p. 788), where all parties have the same information on the distribution of a technological parameter, with the state of nature being unknown at the contracting stage.

\section{The Model}

In this section we draw on the industry characteristics described above to construct a formal model. There are two related research and development (R\&D) stages in an intermediate market for technology, and a final product market. Upstream, a for-profit independent unit (hereafter, "the lab") conducts R\&D activities. Downstream, two firms can outsource R\&D to the lab, and/or also conduct in-house R\&D operations, before competing in the final market where they supply substitutable products.

Information - The two risk-neutral firms (principals) know the strategies available to the other players and the related payoffs, while the risk-neutral lab (an agent) needs not know the downstream cost and demand conditions. Technological uncertainty is described by a parameter $\theta \in \Theta$ whose

\footnotetext{
${ }^{15}$ For example, in Bhattacharya and Guriev (2006) an external unit can sell its technology to one of two client firms, then secretly to a competitor. In Bhattacharya and Guriev (2013) the external unit also chooses a non-verifiable research effort that conditions the value of the technology needed for downstream firms to innovate.

${ }^{16}$ Moreover, "the identities of partners and descriptions of alliances figure prominently in biotechnology companies' securities registration statements" when an initial public offering is in preparation (Stuart et al., 1999, p. 327).
} 
distribution is known by all parties, with the state of nature being unknown ex-ante (at the contracting stage, that is before the lab tests technological options) but observable and verifiable ex-post (so that payments can be made contingent on it and the firms are committed to their contracts). ${ }^{17}$.

Profit functions - The non-negative external R\&D levels, as chosen by the lab specifically for each firm, are described by $\mathbf{x}=\left(x_{1}, x_{2}\right)$. The internal $\mathrm{R} \& \mathrm{D}$ levels and the final-market commercial strategies, as non-cooperatively chosen by the firms, are described by $\mathbf{y}=\left(y_{1}, y_{2}\right)$ and $\mathbf{z}=\left(z_{1}, z_{2}\right)$, respectively. ${ }^{18}$

The lab's net profit is

$$
v_{0}(\mathbf{x}, \theta)=t_{1}(\mathbf{x}, \theta)+t_{2}(\mathbf{x}, \theta)-f_{0}(\mathbf{x}, \theta)
$$

where $f_{0}$ is the lab's cost, and $t_{i}$ is firm $i^{\prime}$ s transfer payment, both functions of $\mathbf{x}$ and $\theta$. As a transfer can include an upfront part, it can be rewritten as $t_{i}(\mathbf{x}, \theta)=\underline{t}_{i}+k_{i}(\mathbf{x}, \theta)$, where the function $k_{i}$ formalizes the fine tuning of each firm's contingent transfer payment with the technology effectively supplied by the lab (after uncertainty is realized), as made possible by complex non-compete clauses and a well-functioning legal environment (see section 2).

Each firm $i^{\prime}$ s net profit is

$$
v_{i}(\mathbf{x}, \mathbf{y}, \mathbf{z}, \theta)=g_{i}\left(x_{i}+y_{i}, x_{j}, y_{j}, \mathbf{z}, \theta\right)-f_{i}\left(y_{i}, \theta\right)-t_{i}(\mathbf{x}, \theta)
$$

$i, j=1,2, j \neq i$, where $f_{i}$ is the firm-specific cost of generating $y_{i}$ internally, and $g_{i}$ is a gross profit function. In the latter function, firm $i$ 's external and internal R\&D levels $x_{i}$ and $y_{i}$ are added as an argument, which formalizes the technological assumption that all R\&D tasks can be $a$ priori performed either externally or internally. However, the respective equilibrium values are the outcome of distinct decision processes that can result in external and internal efforts being substitutes or complements ( $y_{i}^{*}$ can be either decreasing or increasing in $x_{i}$ in Proposition 1, below). The competitor's variables $x_{j}$ and $y_{j}$ are arguments of the same function, allowing for technological spillovers received indirectly through the lab and directly from the competitor. The gross profit also depends on final-market noncooperative strategies, $\mathbf{z}$. (Several examples are discussed below that adapt specific algebraic forms from the R\&D literature, with a focus on the effect of uncertainty in Examples 2 and 3.)

Timing - There are four stages, as follows:

\footnotetext{
${ }^{17} \mathrm{R} \& \mathrm{D}$ contracts usually include provisions for dispute resolution and point to an external private arbitrager, or to a specific Court, in case of litigation (e.g., Robinson and Stuart, 2007).

${ }^{18}$ The argument $\mathbf{z}$ can represent prices or quantities, indifferently, or refer to more elaborate competitive interactions.
} 
(i) The two firms simultaneously and non-cooperatively choose a transfer function $t_{i}(\mathbf{x}, \theta) \geq 0$, $i=1,2$, offered to the lab as a contract.

Each contract offer thus connects a payment to the lab's chosen R\&D levels for any possible state of nature, and the specification that payments are non-negative formalizes the assumption that the institutional environment allows the firms to reduce payments but not to impose penalty fees to the lab in cases of unsuccesful outcomes. More specifically, in what follows, we assume that both $\underline{t}_{i}$ and $k_{i}(\mathbf{x}, \theta)$ are non-negative.

(ii) The lab accepts either both contract offers simultaneously, or only one, or none, and chooses the firm-specific R\&D levels in $\mathbf{x}$ that maximize its expected profit $E_{\theta}\left[v_{0}(\mathbf{x}, \theta)\right]$.

If accepted contracts include an upfront payment, the lab receives $\underline{t}_{i}$ from each contracting firm.

At this stage the lab refuses all contracts if they imply a lower benefit than the reservation value $\underline{v}_{0}=0$, and it takes only one of the two contracts if this implies a higher benefit than accepting the two offers. ${ }^{19}$ Formally, for any given $t_{j}$ offered by firm $j$, the lab accepts firm $i$ 's contract offer only if

$$
E_{\theta}\left[v_{0}(\mathbf{x}, \theta)\right] \geq \sup \left\{0, \max _{\mathbf{x}} E_{\theta}\left[t_{j}(\mathbf{x}, \theta)-f_{0}(\mathbf{x}, \theta)\right]\right\}
$$

for some $\mathbf{x} \geq(0,0), i, j=1,2, j \neq i$. As the firms' contract offers cannot be negative, in equilibrium (3) is always exactly satisfied. ${ }^{20}$ This however does not imply that the equilibrium R\&D levels and transfer functions are symmetric, nor that payments are both positive. It can be the case that firm $i$ offers a "null" contract, where $t_{i}(\mathbf{x}, \theta)=0$, all $(\mathbf{x}, \theta)$, and still receives technology, if for example limiting inter-firm technological spillovers is prohibitively costly for the lab.

(iii) Given the accepted contracts, and on the basis of their expected returns, the firms simultaneously and non-cooperatively choose their own internal $R \& D$ level $y_{i} \geq 0$.

Uncertainty realizes and the state of nature is revealed to all parties.

(iv) Given the realized state of nature, and the outcome of ex-ante chosen R\&D levels, the firms

\footnotetext{
${ }^{19}$ As the lab (an agent) can choose to accept only a subset of contracts offered by the two firms (principals), this is a "delegated common agency" model in the terminology introduced by Bernheim and Whinston (1986a).

${ }^{20}$ Should in equilibrium the for-profit lab contract exclusively with, say, firm 1 , to deliver $\tilde{\mathbf{x}} \in$ $\arg \max _{\mathbf{x}} E_{\theta}\left[t_{1}(\mathbf{x}, \theta)-f_{0}(\mathbf{x}, \theta)\right]$, for any non-negative contract offer $t_{2}$ the lab would earn in expectation $E_{\theta}\left[t_{1}(\tilde{\mathbf{x}}, \theta)-f_{0}(\tilde{\mathbf{x}}, \theta)\right]<E_{\theta}\left[t_{1}(\tilde{\mathbf{x}}, \theta)+t_{2}(\tilde{\mathbf{x}}, \theta)-f_{0}(\tilde{\mathbf{x}}, \theta)\right]$, a contradiction, so (3) is always satisfied. Moreover, should the lab supply $\hat{\mathbf{x}}$ to earn the expected payoff $E_{\theta}\left[t_{1}(\hat{\mathbf{x}}, \theta)+t_{2}(\hat{\mathbf{x}}, \theta)-f_{0}(\hat{\mathbf{x}}, \theta)\right]>E_{\theta}\left[t_{1}(\tilde{\mathbf{x}}, \theta)-f_{0}(\tilde{\mathbf{x}}, \theta)\right]$, then firm 2 would find it profitable to adapt its contract offer to $t_{2}^{\prime}$ verifying $E_{\theta}\left[t_{2}^{\prime}(\hat{\mathbf{x}}, \theta)\right]=E_{\theta}\left[t_{2}(\hat{\mathbf{x}}, \theta)\right]-\varphi$, where $\varphi=E_{\theta}\left[\left(t_{1}(\hat{\mathbf{x}}, \theta)+t_{2}(\hat{\mathbf{x}}, \theta)-f_{0}(\hat{\mathbf{x}}, \theta)\right)-\left(t_{1}(\tilde{\mathbf{x}}, \theta)-f_{0}(\tilde{\mathbf{x}}, \theta)\right)\right]$, and hence $(3)$ holds with equality.
} 
simultaneously and non-cooperatively choose their final-market commercial strategy $z_{i}(\theta) \geq 0$.

The firms transfer their respective contingent payments $k_{i}(\mathbf{x}, \theta)=t_{i}(\mathbf{x}, \theta)-\underline{t}_{i}$ to the lab.

The net profits to the lab and to each firm, after contingent payments have been transferred, are derived from the following equilibrium concept.

Equilibrium concept - For any $(\mathbf{x}, \mathbf{y})$ and any realization of $\theta$, henceforth we assume that (i) there exists a unique final-market Nash equilibrium $\mathbf{z}^{*}(\mathbf{x}, \mathbf{y}, \theta)$, and (ii) for any $\mathbf{x}$, and given the distribution of $\theta$, there exists a unique internal-R\&D stage Nash equilibrium $\mathbf{y}^{*}(\mathbf{x})$, so that we may introduce

$$
\tilde{g}_{i}(\mathbf{x}, \theta)=g_{i}\left(x_{i}+y_{i}^{*}(\mathbf{x}), x_{j}, y_{j}^{*}(\mathbf{x}), \mathbf{z}^{*}\left(\mathbf{x}, \mathbf{y}^{*}(\mathbf{x}), \theta\right), \theta\right)-f_{i}\left(y_{i}^{*}(\mathbf{x}), \theta\right),
$$

that is firm $i$ 's concentrated profit net of internal R\&D costs. Finally, for any given $\mathbf{t}=\left(t_{1}, t_{2}\right)$, and again given the distribution of $\theta$, we denote by $X(\mathbf{t})$ the set of $R \& D$ choices that maximize the lab's profits in expectation, that is $X(\mathbf{t})=\arg \max _{\mathbf{x}} E_{\theta}\left[v_{0}(\mathbf{x}(\mathbf{t}), \theta)\right]$.

The following definitions are needed before introducing the solution concept:

(1) for any $\mathbf{x} \in X\left(t_{i}, t_{j}\right)$ and $\mathbf{x}^{\prime} \in X\left(t_{i}^{\prime}, t_{j}\right)$, firm $i^{\prime}$ s transfer function $t_{i}$ is $a$ best response to the other firm's $t_{j}$ if $E_{\theta}\left[\tilde{g}_{i}(\mathbf{x}, \theta)-t_{i}(\mathbf{x}, \theta)\right] \geq E_{\theta}\left[\tilde{g}_{i}\left(\mathbf{x}^{\prime}, \theta\right)-t_{i}^{\prime}\left(\mathbf{x}^{\prime}, \theta\right)\right]$, all $t_{i}^{\prime} ;$

(2) the transfer function $t_{i}$ is truthful relative to firm $i^{\prime}$ s payoff with $\mathbf{x}^{o}$ if $t_{i}(\mathbf{x}, \theta)=\sup \left\{0, \tilde{g}_{i}(\mathbf{x}, \theta)-\right.$ $\left.\left[\tilde{g}_{i}\left(\mathbf{x}^{o}, \theta\right)-t_{i}\left(\mathbf{x}^{o}, \theta\right)\right]\right\} .^{21}$

The solution concept is the truthful subgame-perfect Nash equilibrium (TSPNE). The four-tuple $(\tilde{\mathbf{t}}, \tilde{\mathbf{x}}, \tilde{\mathbf{y}}, \tilde{\mathbf{z}})$ is a TSPNE if, for $i, j=1,2, j \neq i:(\mathbf{i}) \tilde{\mathbf{z}}=\mathbf{z}^{*}(\tilde{\mathbf{x}}, \tilde{\mathbf{y}}, \theta)$; (ii) $\tilde{\mathbf{y}}=\mathbf{y}^{*}(\tilde{\mathbf{x}}) ;\left(\right.$ iii) $\tilde{\mathbf{x}} \in X(\tilde{\mathbf{t}}) ;\left(\right.$ iv) $\tilde{t}_{i}$ is a best response to $\tilde{t}_{j}$; and $(\mathrm{v}) \tilde{t}_{i}$ is truthful relative to firm $i^{\prime}$ s payoff with $\tilde{\mathbf{x}}$. It follows that $\tilde{t}_{i}(\mathbf{x}, \theta)=$ $\sup \left\{0, \tilde{g}_{i}(\mathbf{x}, \theta)-v_{i}(\tilde{\mathbf{x}}, \tilde{\mathbf{y}}, \tilde{\mathbf{z}}, \theta)\right\}$, where $v_{i}(\tilde{\mathbf{x}}, \tilde{\mathbf{y}}, \tilde{\mathbf{z}}, \theta)=\left[\tilde{g}_{i}(\tilde{\mathbf{x}}, \theta)-\tilde{t}_{i}(\tilde{\mathbf{x}}, \theta)\right]$ is firm $i^{\prime}$ s equilibrium payoff as a function of the realization of $\theta$.

Truthfulness is a standard refinement in delegated common agency games, and two properties in the theoretical literature offer a strong justification for using it (Bernheim and Whinston, 1986b; Laussel and Le Breton, 2001; Martimort, 2007). A first property is that, for any set of transfer offers by any of the two firms, there exists a truthful strategy in the other firm's best-response correspondence. A

\footnotetext{
${ }^{21}$ When the gross profit $\tilde{g}_{i}(\mathbf{x}, \theta)$ exceeds the firm's expected net profit at $\mathbf{x}^{o}$, that is $\tilde{g}_{i}\left(\mathbf{x}^{o}, \theta\right)-t_{i}\left(\mathbf{x}^{o}, \theta\right)$, the difference between $t_{i}(\mathbf{x}, \theta)$ and $t_{i}\left(\mathbf{x}^{o}, \theta\right)$ is equal to the difference between $\tilde{g}_{i}(\mathbf{x}, \theta)$ and $\tilde{g}_{i}\left(\mathbf{x}^{o}, \theta\right)$; otherwise the transfer $t_{i}(\mathbf{x}, \theta)$ is set equal to zero. For each $\theta$, on its positive part a truthful contract offer thus exactly reflects firm $i$ 's valuation of $\mathbf{x}$ relative to the value for $\mathbf{x}^{O}$.
} 
firm can thus restrict itself to truthful strategies at no cost. A second property is that, when firms can communicate with each other, all truthful Nash equilibria are coalition-proof. Therefore, the two firms' joint net profits in a TSPNE are not lower than in any other subgame-perfect Nash equilibrium. $^{22}$ The proofs of several propositions in Section 4 present the technical challenge of extending fundamental results of this common agency literature to a context where firms not only interact as principals in the intermediate market for external $R \& D$, but also compete in internal technological decisions and in final-market commercial strategies. ${ }^{23}$

Technological assumptions - The results in the next sections refer to properties of the lab's and the two firms' expected costs $\hat{f}_{0}(\mathbf{x})=E_{\theta}\left[f_{0}(\mathbf{x}, \theta)\right]$ and $\hat{f}_{i}(\mathbf{x})=E_{\theta}\left[f_{i}\left(x_{i}, \theta\right)\right], i=1,2$, which are (weakly) increasing in their respective arguments, and to the firms' expected gross profit ${ }^{24} \hat{g}_{i}\left(x_{i}+y_{i}, x_{j}, y_{j}\right)=$ $E_{\theta}\left[g_{i}\left(x_{i}+y_{i}, x_{j}, y_{j}, \mathbf{z}^{*}(\mathbf{x}, \mathbf{y}, \theta), \theta\right)\right], i, j=1,2, j \neq i$. The latter expression is (weakly) increasing in the firm's own R\&D levels received from the lab or sourced internally (formally $\partial \hat{g}_{i} / \partial s_{i} \geq 0$ where $\left.s_{i}=x_{i}+y_{i}, i=1,2\right)$, but can be decreasing, or not, in the rival's arguments $x_{j}$ and $y_{j}$. In any case a firm's expected gross profit is (weakly) more impacted by its own $R \& D$, as either purchased from the lab or produced in-house, than by its rival's arguments:

$$
\begin{gathered}
\frac{\partial \hat{g}_{i}}{\partial x_{i}} \geq\left\|\frac{\partial \hat{g}_{i}}{\partial x_{j}}\right\|, \\
\| \quad, \\
\frac{\partial \hat{g}_{i}}{\partial y_{i}} \geq\left\|\frac{\partial \hat{g}_{i}}{\partial y_{j}}\right\|,
\end{gathered}
$$

$i, j=1,2, j \neq i$. The vertical comparison of the terms on the RHS of the inequality sign in (4) and (5) specifies that the R\&D leaks emanating from the lab are (weakly) more informative than the technological spillovers received from the competitor's internal facilities. ${ }^{25}$

For both firms, returns to R\&D can be either non-increasing (that is, $\partial^{2} \hat{g}_{i} / \partial s_{i}^{2} \leq 0, i=1,2$ ), or in-

\footnotetext{
${ }^{22} \mathrm{~A}$ Nash equilibrium is coalition-proof if it is robust to credible threats of deviations by any subset of principals (for a formal definition see Bernheim, Peleg, and Whinston, 1987). With two principals only, a coalition-proof equilibrium is Pareto-efficient among principals (Bernheim and Whinston, 1986b). For a discussion on truthfulness as an equilibrium refinement, see Martimort (2007).

${ }^{23}$ In appendix the proofs of Lemmas $A .1$ and $A .2$ establish formal conditions which, in combination with theorems by Laussel and Le Breton (2001) and extensions by Billette de Villemeur and Versaevel (2003), lead to Propositions 2, 3, and 5.

${ }^{24}$ Here we follow Amir et al. (2003) by suggesting that the reduced-form expected gross profit function $\hat{g}_{i}$ can be interpreted as the overall payoff of a multi-stage game in the product market. Then R\&D choices are seen as long-term decisions, on which we focus, followed by a series of short-term final-market decisions.

${ }^{25}$ In the words of Lai, Riezman, and Wang (2009), "information leakage is much more severe in the absence of internal controls when R\&D is outsourced" (p. 487).
} 
creasing. The sign of all partial cross-derivatives can also be either non-positive (that is, $\partial^{2} \hat{g}_{i} / \partial x_{i} \partial x_{j} \leq$ $0, \partial^{2} \hat{g}_{i} / \partial x_{i} \partial y_{j} \leq 0, \partial^{2} \hat{g}_{i} / \partial y_{i} \partial x_{j} \leq 0$, and $\left.\partial^{2} \hat{g}_{i} / \partial y_{i} \partial y_{j} \leq 0, i, j=1,2, j \neq i\right)$, or positive. In all cases, the second-order impact of a firm's R\&D, either produced in-house or received from the lab, on its own expected gross profit, is higher than the second-order effect of its competitor's R\&D:

$$
\begin{aligned}
\left\|\frac{\partial^{2} \hat{g}_{i}}{\partial y_{i} \partial x_{i}}\right\| & \geq\left\|\frac{\partial^{2} \hat{g}_{i}}{\partial y_{i} \partial x_{j}}\right\|, \\
\| & \vee । \\
\left\|\frac{\partial^{2} \hat{g}_{i}}{\partial y_{i}^{2}}\right\| & \geq\left\|\frac{\partial^{2} \hat{g}_{i}}{\partial y_{i} \partial y_{j}}\right\|,
\end{aligned}
$$

$i, j=1,2, j \neq i$. The vertical comparison of cross-derivatives in (6) and (7) indicates that each firm 's marginal expected gross profit is (weakly) more impacted by the technological leakages that emanate from the lab than from its competitor. These technological assumptions are very mild as they bear only on expected profit expressions, and they encompass many possible specifications encountered in the literature (we illustrate with examples of specific algebraic forms in the next section).

Standalone values - The lab can guarantee for itself the value $\underline{v}_{0}=0$ (a normalization). As for the firms, to define their outside option suppose that $j$ has exclusive access to the lab, so that their contractual relationship results in $\mathbf{x}_{j}^{*} \in \arg \max _{\mathbf{x}}\left\{E_{\theta}\left[\tilde{g}_{j}(\mathbf{x}, \theta)-f_{0}(\mathbf{x}, \theta)\right]\right\}$. Then firm $i$ can only rely on internal resources, and it chooses $y_{i}^{*}\left(\mathbf{x}_{j}^{*}\right)$ to earn the standalone expected value $\underline{v}_{i}=E_{\theta}\left[\tilde{g}_{i}\left(\mathbf{x}_{j}^{*}, \theta\right)\right]$. Here $\mathbf{x}_{j}^{*}=\left(x_{i}^{*}, x_{j}^{*}\right)$, with $x_{i}^{*} \geq 0$, so firm $i$ can possibly receive technology, without financial compensation, despite firm $j$ 's exclusive relationship with the lab. For an equilibrium to exist, with technology outsourcing, it must be the case that $v_{i}^{*}=E_{\theta}\left[\tilde{g}_{i}(\tilde{\mathbf{x}}, \theta)-\tilde{t}_{i}(\tilde{\mathbf{x}}, \theta)\right] \geq \underline{v}_{i}$, for both firms. ${ }^{26}$

\section{Technological Conditions and Equilibrium Analysis}

In this section, we investigate the circumstances in which technology outsourcing either reduces or stimulates internal R\&D levels, before deriving conditions for the lab to appropriate a share of R\&D profits, or to exactly break-even to the benefit of the outsourcing firms.

As a first result, we find that in equilibrium each firm's internal R\&D level $y_{i}^{*}$ can be substitutable or a complementary to the level $x_{i}$ received from the lab. The sign of the relation between $y_{i}^{*}$ and $x_{i}$

\footnotetext{
${ }^{26}$ In the proofs of Propositions 2 and 3 we check that this condition holds in equilibrium (see Appendix A.4).
} 
depends on the nature of R\&D returns, but not on technological spillovers.

Proposition 1 (external/internal R\&D) The equilibrium level of a firm's internal RED activity $y_{i}^{*}$ is decreasing in the contracted external lab's activity $x_{i}$ if and only if the gross profit functions $\hat{g}_{i}$ have decreasing returns in $s_{i}=x_{i}+y_{i}, i=1,2$. More formally:

$$
\frac{d y_{i}^{*}}{d x_{i}} \lesseqgtr 0 \Leftrightarrow \frac{\partial^{2} \hat{g}_{i}}{\partial s_{i}^{2}} \lesseqgtr 0 .^{27}
$$

A first message in this proposition is that whether contracted-out R\&D reduces or raises internal activity does not depend on inter-firm technological spillovers, because the second-order effect in (8) bears only on each firm $i$ 's own argument $s_{i}$, not on $x_{j}$ or $y_{j}, i, j=1,2, j \neq i$. This property contrasts with the well-known lesson received from many papers that adopt the analytical framework of d'Aspremont and Jacquemin (1988) in order to focus on horizontal technological interactions. In these papers, the strategic substitutability or complementarity of the firms' technological choice variables depends entirely on whether a spillover parameter is low or high, respectively. In our model, the firms also interact vertically by competing in their contract offers to the external unit. This vertical interaction appears to dominate the horizontal effects for what regards the substitutability/complementarity outcome.

Another message in Proposition 1 is that the relationship between external and internal sourcing is formally ambiguous. This ambiguity is structural, in that it depends on the functional form of firms' gross profit. Here contracted-out R\&D reduces internal activity if and only if there are decreasing returns to the introduction of a new technology in downstream operations. In real-world circumstances, returns to R\&D in the biopharmaceutical domain depend on a number of factors, including the therapeutic area (for example, the discovery of new antibiotics does not present the same challenges as the discovery of a universal influenza vaccine) or the relative exploratory nature of a scientific approach (cell therapies against cancer are only emerging, unlike chemotherapy). Our proposition predicts that situations of decreasing returns imply more concentration of R\&D activities than with increasing returns, either upstream in external units or downstream in pharma companies.

This result is reminiscent of several recent empirical analyses that indicate a context-specific relationship between external and internal R\&D sources in the biopharmaceutical industry. In Hage-

\footnotetext{
${ }^{27}$ More specifically, $\frac{d y_{i}^{*}}{d x_{i}}=0$ if and only if either (i) $\frac{\partial^{2} \hat{g}_{i}}{\partial s_{i}^{2}}=0$, or (ii) $\frac{\partial^{2} \hat{g}_{i}}{\partial x_{i}^{2}}=\frac{\partial^{2} \hat{g}_{i}}{\partial x_{i} \partial y_{j}}<0, \frac{\partial^{2} \hat{g}_{j}}{\partial x_{j}^{2}}=\frac{\partial^{2} \hat{g}_{j}}{\partial x_{j} \partial x_{j}}<0$, and $\frac{\partial^{2} \hat{f}_{j}}{\partial y_{j}^{2}}=0$, where $i, j=1,2, j \neq i$ (see Appendix A.2).
} 
doorn and Wang (2012) the estimated sign of the marginal effect of internal R\&D expenditure on the innovative output is negative, with internal and external R\&D turning out to be substitutes only at lower levels of in-house R\&D investments. In Ceccagnoli et al. (2014), the estimated sign of the partial cross-derivative of an innovation production function with respect to external and internal R\&D expenditure is found to depend on a series of factors. These empirical investigations and our formal characterization share the conclusion that external and internal R\&D are neither complements not substitutes per se, the exact connection between the two channels being rather context related, as captured here by the sign of a second-order effect.

In what follows we build on Proposition 1 by first considering separately situations of nonincreasing returns $\left(\partial^{2} \hat{g}_{i} / \partial s_{i}^{2} \leq 0\right)$, before discussing the robustness of our results when we shift to non-decreasing returns $\left(\partial^{2} \hat{g}_{i} / \partial s_{i}^{2} \geq 0\right)$. In either cases, to characterize the distribution of R\&D profits among the intermediate R\&D market participants we need defining as a value function the highest expected joint profit for the lab together with any subset of firms, that is

$$
v(S)=\max _{\mathbf{x}}\left(\sum_{i \in S} E_{\theta}\left[\tilde{g}_{i}(\mathbf{x}, \theta)-f_{0}(\mathbf{x}, \theta)\right]\right)
$$

where $S \in\{\varnothing,\{1\},\{2\},\{1,2\}\}$.

We assume that $v(\varnothing)=\underline{v}_{0}=0$, which describes the no contract situation, and that $v(\{i\}) \geq \underline{v}_{i}$, implying that firm $i$ 's exclusive control of the lab dominates its standalone value, $i=1,2$. Hereafter, for conciseness we denote the maximum expected industry profit $v(\{1,2\})$ by $\Lambda$.

The value function $v($.$) in (9) is instrumental for the caracterization of equilibrium outcomes in$ the intermediate market for technology, as it captures the interplay of indirect and direct technological externalities: there are indirect technological externalities if the lab's cost $f_{0}$ of conducting firm-specific R\&D tasks is characterized by economies or diseconomies of scope; there are direct externalities if the R\&D received or generated by firm $i$ enters in the gross profit function $\hat{g}_{j}$ of its competitor, $i, j=1,2, j \neq i{ }^{28}$ Indirect (through the lab) and direct (inter-firm) technological externalities can differ in magnitude and in sign, and an aggregate measure of the combination of both categories

\footnotetext{
${ }^{28}$ In (9), recall that $\tilde{g}_{i}(\mathbf{x}, \theta)=g_{i}\left(x_{i}+y_{i}^{*}(\mathbf{x}), x_{j}, y_{j}^{*}(\mathbf{x}), \mathbf{z}^{*}\left(\mathbf{x}, \mathbf{y}^{*}(\mathbf{x}), \theta\right), \theta\right)-f_{i}\left(y_{i}^{*}(\mathbf{x}), \theta\right)$, so that firm $i^{\prime}$ s profit does not depend only on the R\&D generated by the rival internally $\left(y_{j}^{*}(\mathbf{x})\right)$, but also on the technology received by the latter firm from the lab $\left(x_{j}\right)$.
} 
of externalities is given by the structural parameter

$$
\epsilon=\Lambda-v(\{1\})-v(\{2\})
$$

If $\epsilon<0$, that is $v($.$) is strictly subadditive, the maximization in \mathbf{x}$ of joint profits generates less value than the sum of individual profits as obtained by each firm when it exclusively controls the lab, a situation where negative externalities dominate. Otherwise $v($.$) is superadditive, and positive$ externalities (weakly) dominate.

Non-increasing returns to $R \& D$. In this section we assume that

$$
\frac{\partial^{2} \hat{g}_{i}}{\partial s_{i}^{2}} \leq 0
$$

$i=1,2$. Simple sufficient conditions on the primitives of the model can now be derived that determine the sign of the aggregate measure of externalities, and whether the equilibrium expected industry profit is fully appropriated by the two firms or partly retained by the lab. These conditions bear on the sign of indirect and direct R\&D externalities, hence on the lab's expected costs $\hat{f}_{0}(\mathbf{x})$ and firms' expected gross profits $\hat{g}_{i}\left(x_{i}+y_{i}, x_{j}, y_{j}\right)$, respectively.

Non-negative RED externalities - Suppose, as a first case, that indirect and direct R\&D externalities are non-negative. Formally, for $i, j=1,2, j \neq i$,

$$
\begin{gathered}
\frac{\partial^{2} \hat{f}_{0}}{\partial x_{i} \partial x_{j}} \leq 0 \\
\frac{\partial \hat{g}_{i}}{\partial x_{j}} \geq 0, \quad \frac{\partial \hat{g}_{i}}{\partial y_{j}} \geq 0 .
\end{gathered}
$$

Proposition 2 (non-negative R\&D externalities) Conditions (12-13) imply that $\epsilon \geq 0(v($.$) is superad-$ ditive). Then there exists a continuum of firm equilibrium expected payoffs $\left(v_{1}^{*}, v_{2}^{*}\right) \geq\left(\underline{v}_{1}, \underline{v}_{2}\right)$ that verify

$$
v_{1}^{*}+v_{2}^{*}=\Lambda,
$$

and the lab exactly breaks even in expectation, that is

$$
v_{0}^{*}=0 .
$$


In (12) the non-positive sign of the cross-derivatives of $\hat{f}_{0}$ in the dimensions of $\mathbf{x}$ describes economies of scope in the production of R\&D inside the external lab. Selecting a higher $x_{i}$, as demanded by firm $i$, makes it less costly for the lab to satisfy firm $j .{ }^{29}$ This condition is consistent with empirical investigations that evidence the presence of economies of scope mainly in drug discovery (Henderson and Cockburn, 1996) and only to some extent in clinical trials (Cockburn and Henderson, 2001). ${ }^{30}$

For an interpretation of the conditions in (13), recall from the structure of each firm's gross profit function in (2) that R\&D decisions generate not only inter-firm technological spillovers $\left(x_{j}\right.$ and $y_{j}$ are arguments of $g_{i}, j \neq i$ ) but also a product-market rivalry effect (firm $j$ 's external and internal $\operatorname{R\& D}$ impacts firm $i$ 's strategy $z_{i}, j \neq i$ ). As the two non-negative derivatives in (13) relate to the reduced-form $\hat{g}_{i}$ of the gross profit expression, they capture situations where technological spillovers dominate the negative business stealing effect. This specification points to situations of substantial spillovers, as observed by Henderson and Cockburn (1996) between pharmaceutical firms. It is also consistent with Bloom et al. (2013) where significant technological spillovers are found in the case of pharmaceuticals, together with strategic complementarity in R\&D (for a formal illustration see Example 4 below, case $\beta \geq 1 / 2$ ).

Examples of complementarities in pre-clinical research include the use of mRNA (messenger ribonucleic acid) to produce medicines that code for the production of proteins by cells in the body, as the same biological mechanism can lead in principle to the treatment of many different diseases. The same applies to research activities in the immuno-oncology domain, as the understanding of how immune responses are controlled by "checkpoints" can result in drugs against a large set of different targets. $^{31}$ In that domain, a molecule approved for the treatment of a given type of cancer can be extended to other indications - a kind of market expansion effect - with competing pharma companies learning from each other the indications toward which their respective research efforts can be targeted.

Non-negative indirect and direct externalities reflect circumstances of weak technological rivalry among the two firms, both in their contract offers to the lab and in their internal operations, implying

\footnotetext{
${ }^{29}$ As Pisano (2006a) puts it, "knowledge and capabilities accumulated in the pursuit of one therapeutic area can often be leveraged to others" (p. 101).

30 "It may be the case ... that success in drug development is purely a function of success in drug discovery"(Cockburn and Henderson, 2001, p. 1053).

31 "The modulation of immune checkpoints using monoclonal antibodies can have a universal effect on immune responses that is not dependent on tumour histologies or individual cancer-specific antigens" (Hoos, 2016, p. 235).
} 
a limited ability of the external lab to appropriate R\&D benefits. This theoretical characterization is consistent with the empirical observation that the average profitability of biotech units is persistently low. Proposition 2 actually establishes that the two firms appropriate all industry profits, and in expectation the lab exactly breaks even. Therefore:

Corollary 1 When conditions (12-13) hold, incentives to invest upstream, in the external unit, are delinked from the value generated by $R \mathcal{E} D$, to the exclusive benefit of downstream sponsors.

The following (deterministic) example illustrates Propositions 1 and 2 with specific cost and demand functional forms borrowed from the R\&D literature.

Example $1 \square$ Each firm i's inverse demand is $p_{i}(\mathbf{q})=S\left(1-\frac{2 q_{i}}{u_{i}^{2}}-\frac{\sigma}{u_{i}} \frac{q_{j}}{u_{j}}\right), i, j=1,2, j \neq i$, where $S$ is the number of identical consumers, $\sigma \in(0,2)$ captures horizontal product differentiation, and $u_{i}$ measures product quality (e.g., increased drug efficacy), as in Symeonidis (2003). Specifically, let $u_{i}=\varepsilon\left(s_{i}\right)^{1 / 4}+\varepsilon \beta\left(s_{j}\right)^{1 / 4}$, where $\varepsilon>0$ is an inverse cost measure, $\beta \in[0,1]$ is an inter-firm technological parameter, $s_{i}=x_{i}+y_{i}$ and $s_{j}=x_{j}+y_{j}, i, j=1,2, j \neq i .^{32}$ For simplicity, we set $S=\sigma=\varepsilon=1, \beta=1 / 2$, and the production cost to zero, before solving for the Cournot-Nash quantities $\left(q_{1}^{*}(\mathbf{x}, \mathbf{y}), q_{2}^{*}(\mathbf{x}, \mathbf{y})\right)$. Inserting the latter expressions in $g_{i}\left(s_{i}, x_{j}, y_{j}, \mathbf{q}\right)=p_{i}(\mathbf{q}) q_{i}$ leads to $\hat{g}_{i}\left(s_{i}, x_{j}, y_{j}\right)$. As $\partial^{2} \hat{g}_{i} / \partial s_{i}^{2}<0$ (decreasing returns) for all $s_{i}>0$, from Proposition 1 we have $d y_{i}^{*} / d x_{i}<0$ (substitutability). Moreover $\partial \hat{g}_{i} / \partial x_{j}>0$ and $\partial \hat{g}_{i} / \partial y_{j}>0$ (positive direct externalities) for all $x_{i}, x_{j}>0$, so (13) is satisfied. ${ }^{33}$ Then any additive cost function for the lab (e.g., $\hat{f}_{0}(\mathbf{x})=x_{1}+x_{2}$ ) satisfies (12), in which case from Proposition 2 the downstream firms appropriate the industry profit.

Negative RED externalities - Suppose now that indirect and direct R\&D externalities are negative, that is for $i, j=1,2, j \neq i$,

$$
\begin{gathered}
\frac{\partial^{2} \hat{f}_{0}}{\partial x_{i} \partial x_{j}}>0, \\
\frac{\partial \hat{g}_{i}}{\partial x_{j}} \leq 0, \quad \frac{\partial \hat{g}_{i}}{\partial y_{j}} \leq 0 .
\end{gathered}
$$

Proposition 3 (negative R\&D externalities) Conditions (16-17) imply that $\epsilon<0(v($.$) is strictly subad-$ ditive). Then there is a unique pair of firm equilibrium expected payoffs $\left(v_{1}^{*}, v_{2}^{*}\right)$, which are

$$
v_{i}^{*}=v(\{i\})-|\epsilon| \geq \underline{v}_{i}
$$

\footnotetext{
${ }^{32}$ If $x_{1}=x_{2}=0$ we have the same specification as in Symeonidis (2003), where the functional form for $u_{i}$ is adapted from Motta (1992).

${ }^{33}$ The expressions of derivatives are omitted for space limitation. They are available from the authors on request.
} 
$i=1,2$, and the lab appropriates a share of expected industry profits

$$
v_{0}^{*}=|\epsilon|>0 .
$$

The condition on $f_{0}$ in (16) formalizes a case of congestion, or diseconomies of scale, in the production of R\&D by the external lab. Supplying more R\&D to a given firm makes it more costly to serve the other firm. The conditions on $\hat{g}_{i}$ in (17) describe circumstances in which more of firm $j$ 's R\&D, as sourced externally or produced internally, weakly reduces firm $i$ 's reduced-form gross profit, all other things remaining equal. Together, these formal conditions relate to real-world circumstances that strongly differ from the ones captured by conditions (12-13). Unlike the empirical evidence mentioned in the previous section on early-stage discovery activities that involve biotech entities, diseconomies of scope (Macher and Boerner, 2006) and nonexistent technological spillovers (Danzon et al. 2005; Macher and Boerner, 2006) have been found in later-stage development activities, notably in phase 2 and phase 3 clinical trials, which involve CROs. The formal conditions in (16) and (17) thus point to these development activities.

To illustrate, recent advances in immuno-oncology have induced clinical trials in the hundreds that test new antibodies or combinations (Tang et al., 2018), implying that the enrollment of patients by a CRO for several competing sponsors has become problematic. Moreover, regulatory constraints such as data exclusivity - i.e., a firm that applies for marketing approval cannot refer to the clinical data generated by a competitor over a given time period - entail limited inter-firm spillovers.

In such cases of negative indirect and direct externalities, the client firms compete for the control of the lab's operations in the intermediate R\&D market, and are also penalized by the in-house activity of their competitor. These circumstances are favorable to the lab. Proposition 3 establishes that negative externalities fully drive the distribution of R\&D benefits. Unlike the payoff in the previous section, the lab here appropriates a positive share of industry profits, in direct proportion to $-\epsilon$ which is positive. Each firm's payoff is equal to $v(\{i\}), i=1,2$, as would be earned by controlling the lab exclusively, truncated by $|\epsilon|$. The latter payoff can be shown to be greater that the standalone value $\underline{v}_{i}{ }^{34}$ The theoretical outcome that the external unit extracts a positive share is consistent with the observation that CROs involved in clinical trials, unlike biotech units, on average earn superior average financial returns.

\footnotetext{
${ }^{34}$ See Appendix A.4. From (10) the payoff to the lab can be rewritten as firm $i$ 's marginal contribution to industry profit, that is $v_{i}^{*}=\Lambda-v(\{j\}), i, j=1,2, j \neq i$.
} 
The conditions used in Proposition 3, rewritten in discrete form, apply in the following example. ${ }^{35}$

Example $2 \square$ Assume that $\mathbf{x}, \mathbf{y} \in\{0,1\}^{2}$, so the decision to invest in a cost-reducing program implies a lump-sum expenditure. The lab's $R \mathcal{E} D$ costs are $\hat{f}_{0}(0,0)=0, \hat{f}_{0}(0,1)=\hat{f}_{0}(1,0)=1$, and $\hat{f}_{0}(1,1)=+\infty$, so that the discrete form of condition (16) is satisfied. Here anti-complementarities imply that the lab serves at most one firm profitably. ${ }^{36}$ Firm i's internal $R \mathcal{E} D$ costs are $\hat{f}_{i}\left(y_{i}\right)=\gamma y_{i}$, with $\gamma \geq 1$ capturing a relative inefficiency vis-à-vis the lab (cost cutting is a driver of the use of contract service providers). The unit cost of production is a positive constant $c_{i}\left(x_{i}+y_{i}\right)$, with $c_{i}(0)=c_{H}$ and $0 \leq c_{i}(1)=c_{i}(2)=c_{L}<c_{H}$. The two firms sell a homogeneous good, with total demand $q=\sup \{0, a-p\}$, with $p \geq 0$ and $a>c_{H}$. Given $(\mathbf{x}, \mathbf{y})$, defining $\pi=\left(c_{H}-c_{L}\right)\left(a-c_{H}\right)$, and solving for Bertrand-Nash prices, leads to $\hat{g}_{i}\left(x_{i}+y_{i}, x_{j}, y_{j}\right)=\pi>0$ if $x_{i}+y_{i} \geq 1$ and $x_{j}+y_{j}=0$, and $\hat{g}_{i}\left(x_{i}+y_{i}, x_{j}, y_{j}\right)=0$ otherwise, so the discrete form of condition (17) is also satisfied. We assume that internal $R \mathcal{E} D$ is worth undertaking, that is $\gamma / \pi<1$. To compute equilibrium payoffs, we consider the following two cases: (1) If the lab is inactive $\left(x_{1}=x_{2}=0\right)$, there exists a unique Nash equilibrium in mixed strategies $\left(\alpha_{i}^{*}, \alpha_{j}^{*}\right)$ of internal RED investments, verifying

$$
\alpha_{j}^{*} \times(-\gamma)+\left(1-\alpha_{j}^{*}\right) \times(\pi-\gamma)=\alpha_{j}^{*} \times 0+\left(1-\alpha_{j}^{*}\right) \times 0,
$$

$i, j=1,2, j \neq i$. By symmetry, ${ }^{37} \alpha_{i}^{*}=\alpha_{j}^{*}=1-\frac{\gamma}{\pi}$, which leads to the payoff $v_{i}\left(\alpha_{1}^{*}, \alpha_{2}^{*}\right)=\underline{v}_{i}=0, i=1,2 .{ }^{38}$ So the firms are willing to transact with the lab. (2) When the lab is active, from the assumption on $\hat{f}_{0}$ only one firm is served $\left(x_{i}=1>x_{j}=0\right)$, and no firm invests in internal $R \mathcal{E} D$ since $\hat{g}_{i}\left(1+1,0, y_{j}\right)-\hat{f}_{i}(1)<\hat{g}_{i}(1+$ $\left.0,0, y_{j}\right)-\hat{f}_{i}(0)$, all $y_{j}$, and $\hat{g}_{j}\left(0+1,1, y_{i}\right)-\hat{f}_{j}(1)<\hat{g}_{j}\left(0+0,1, y_{i}\right)-\hat{f}_{j}(0)$, all $y_{i}$. Therefore, industry value is $v(\{1,2\})=v(\{1\})=v(\{2\})$, so that $v_{0}^{*}=v(\{1,2\})=\pi-1$, and $v_{i}^{*}=v(\{1,2\})-v(\{j\})=0$, from Proposition 3. Firms' interests are so antagonistic in this example as to make the lab fully appropriate industry value.

\footnotetext{
${ }^{35}$ In the whole paper differentiability is adopted for notational convenience, but is not required as illustrated by Example 2. So the condition in (16) can be rewritten in discrete form as $\hat{f}_{0}\left(\mathbf{x} \wedge \mathbf{x}^{\prime}\right)+\hat{f}_{0}\left(\mathbf{x} \vee \mathbf{x}^{\prime}\right)-\hat{f}_{0}(\mathbf{x})-\hat{f}_{0}\left(\mathbf{x}^{\prime}\right) \geq 0$, all $\mathbf{x}, \mathbf{x}^{\prime}$, with a strict inequality whenever $\mathbf{x}$ and $\mathbf{x}^{\prime}$ cannot be compared with respect to $\geq$ (strict supermodularity). The conditions in (17) can also be rewritten as $\hat{g}_{i}\left(x_{i}+y_{i}, x_{j}, y_{j}\right) \geq \hat{g}_{i}\left(x_{i}+y_{i}, x_{j}^{\prime}, y_{j}^{\prime}\right)$ for all $\left(x_{j}^{\prime}, y_{j}^{\prime}\right) \geq\left(x_{j}, y_{j}\right)$. In Appendix A.4 the proofs of Propositions 2 and 3 are written for any $\hat{f}_{0}$ which is either weakly submodular or strictly supermodular, respectively.

${ }^{36}$ The cost specification in this example is borrowed from Laussel and Le Breton (2001). The extreme supermodularity of $\hat{f}_{0}$, with $\hat{f}_{0}(1,1)=+\infty$, here helps illustrating the effect of negative externalities on the distribution of industry profits.

${ }^{37}$ Given that in this example firms are assumed to be symmetric, we leave aside the two asymmetric equilibria in pure strategies $\left(y_{1}^{*}=0, y_{2}^{*}=1\right)$ and $\left(y_{1}^{*}=1, y_{2}^{*}=0\right)$.

${ }^{38}$ In this example, when firm $i$ does not participate in the R\&D market, its rival $j$ receives technology from the relatively more efficient lab (so $\left.x_{j}^{*}=1\right)$, exclusively so $\left(x_{i}^{*}=0\right)$, and finds it profitable not to operate internally $\left(y_{j}^{*}=0\right)$. Then in this Bertrand context firm $i$ maximizes profits by not investing in internal R\&D, and its standalone value is $\underline{v}_{i}=\hat{g}_{i}(0+$ $\left.y_{i}^{*}, 1,0\right)-\hat{f}_{i}\left(y_{i}^{*}\right)=\hat{g}_{i}(0+0,1,0)-\hat{f}_{i}(0)=0, i, j=1,2, j \neq i$.
} 
Uncertainty - The structural conditions in Propositions 2 and 3 also capture circumstances in which R\&D outcomes are uncertain for all parties. To see that, consider again Example 2, but with the lab and the firms being successful in R\&D with probability $\theta<1$. The unit cost of production $c\left(x_{i}+y_{i}\right)$ is now $c(0)=c_{H}$ with certainty, $c_{i}(1)=c_{L}$ with probability $\theta$ and $c_{i}(1)=c_{H}$ with probability $1-\theta$, and $c_{i}(2)=c_{L}$ with probability $1-(1-\theta)^{2}$ and $c_{i}(2)=c_{H}$ with probability $(1-\theta)^{2}$. The distribution of (un)favorable events is assumed to be common knowledge ex ante, and the true state is discovered only through the realization of R\&D tasks (between stages (iii) and (iv) in the timing described in Section 3) so firms' payments can be made contingent on it. Condition (16) remains unchanged, and although the process is now uncertain, condition (17) also remains valid in expectation, as the choice of firm $j$ to attempt to innovate always reduces firm $i^{\prime}$ s expected profit. Thus Proposition 3 still holds. We assume as above that R\&D is worth undertaking, even internally, that is $\gamma /(\theta \pi)<1$. Provided that the probability of success $\theta$ remains sufficiently close to 1 so that $1-\theta<\gamma /(\theta \pi)$, again a firm will not engage in R\&D if its rival receives technology from the lab or sources it internally, and we can directly generalise the baseline example: (1) If the lab is inactive $\left(x_{1}=x_{2}=0\right)$, there exists a unique symmetric Nash equilibrium in mixed strategies

$$
\alpha_{i}^{*}(\theta)=\alpha_{j}^{*}(\theta)=\frac{1}{\theta}\left(1-\frac{\gamma}{\theta \pi}\right)
$$

for a payoff which again is the same as the standalone value $\underline{v}_{i}(\theta)=0, i=1,2$, so the firms have an incentive to transact with the lab. (2) When the lab is active, again from the assumption on $\hat{f}_{0}$ only one firm is served $\left(x_{i}=1>x_{j}=0\right)$, and limited uncertainty does not modify the outcome that no firm invests internally since expected net profits verify $\hat{g}_{i}\left(1+1,0, y_{j}\right)-\hat{f}_{i}(1)<\hat{g}_{i}\left(1+0,0, y_{j}\right)-\hat{f}_{i}(0)$, all $y_{j}$, and $\hat{g}_{j}\left(0+1,1, y_{i}\right)-\hat{f}_{j}(1)<\hat{g}_{j}\left(0+0,1, y_{i}\right)-\hat{f}_{j}(0)$, all $y_{i}$. The payoff to the firm that does not receive external R\&D is nil, so that $v(\{1,2\})=v(\{1\})=v(\{2\})$. Then $v_{0}^{*}=v(\{1,2\})$, and $v_{i}^{*}=v(\{1,2\})-v(\{j\})=0, i, j=1,2, j \neq i$, from Proposition 3. With these specifications, the lab's expected equilibrium payoff is $\theta \pi-1$, the firm that benefits from the lab's output exactly breaks even, and its rival earns its standalone value.

In the next example, we show that our results also apply to the polar situation with highly uncertain R\&D. We obtain that, when the competitor or its external contractor might fail with a high probability, it can be a dominant strategy for the firms to engage in R\&D as well.

Example $3 \square$ We consider the specifications of Example 2 with the extension to uncertain RED as above, and 
focus on the case of rare successful outcomes. Internal $R \mathcal{E} D$ can be profitable, that is $\gamma /(\theta \pi)<1$, although $\theta$ is sufficiently close to 0 for $\gamma /(\theta \pi)<(1-\theta)^{2}$ to hold. In this case, the likelihood that the competitor and the lab succeed in RED is so low as to make the probability of simultaneous success negligible. Then everything happens as if, when deciding to engage or not in $R \mathcal{E} D$, each firm were focusing on its own probability of success only, abstracting from the other players' actions. Investing in internal RED is a dominant strategy: (1) If the lab is inactive $\left(x_{1}=x_{2}=0\right)$, the firms' expected payoff is $(1-\theta) \theta \pi-\gamma$, which is slightly higher than the standalone level $\underline{v}_{i}(\theta)=(1-\theta)^{2} \theta \pi-\gamma, i=1$, 2. (2) When the lab is active, again the form of $\hat{f}_{0}$ implies that only one firm is served $\left(x_{i}=1>x_{j}=0\right)$. Still the distinctive feature here, in comparison to the previous example, is that both firms choose to invest internally as well: $\hat{g}_{i}\left(1+1,0, y_{j}\right)-\hat{f}_{i}(1)>\hat{g}_{i}(1+$ $\left.0,0, y_{j}\right)-\hat{f}_{i}(0)$, all $y_{j}$, and $\hat{g}_{j}\left(0+1,1, y_{i}\right)-\hat{f}_{j}(1)>\hat{g}_{j}\left(0+0,1, y_{i}\right)-\hat{f}_{j}(0)$, all $y_{i}$, for $i, j=1,2, j \neq i$. Thus $v(\{i\})=\left(1-(1-\theta)^{2}\right)(1-\theta) \pi-\gamma-1$ (i.e., $\pi$ is earned by firm $i$ when the latter player and the lab do not both fail while firm $j$ fails) and the expected industry value is now $v(\{1,2\})=(3-2 \theta)(1-\theta) \theta \pi-$ $2 \gamma-1$ (i.e., the sum of firm i's expected gross payoff $\left(1-(1-\theta)^{2}\right)(1-\theta) \pi$ and of firm j's gross payoff $(1-\theta)^{2} \theta \pi$ net of total $R \mathcal{E} D$ costs). Therefore, from Proposition 3 we have $v_{0}^{*}=(1-\theta) \theta \pi-1>0$, and $v_{i}^{*}=v(\{1,2\})-v(\{j\})=(1-\theta)^{2} \theta \pi-\gamma$, which is positive but only equal to the standalone level (the profit earned when the competitor controls the lab to its exclusive benefit). By competing for the lab's resources, here the firms earn less than if the lab does not exist.

There is more in Examples 2 and 3 than an illustration of the applicability of a theoretical proposition to specific algebraic forms. Only in the latter example, where the probability of success of R\&D operations is assumed to be low, both the lab and its sponsor engage in R\&D efforts. This outcome is consistent with the empirical evidence (Guedj, 2005) that projects with a low probability of success are more often conducted through a contractual alliance between a large firm and a smaller biotech company (as in Example 3) than conducted entirely within the same entity (as in Example 2). The comparison of Examples 2 and 3 thus rationalizes the general observation that the reduction in drug R\&D productivity over the last decades - which is formally captured here by a lower probability of success - has coincided with increasingly frequent situations where large pharma firms and smaller external biotech units contribute jointly to research and development (Pisano, 2006a; Rydzewski, 2008; Scannell et al., 2012). This is a sufficiently high level of uncertainty, in our theoretical framework, that triggers an investment by all industry participants.

Another interesting equilibrium property illustrated by examples 2 and 3 is that the firms (princi- 
pals), whose payments to the lab (agent) are truthful, are shielded from the uncertainty that is specific to external R\&D operations. The lab, however, bears the risk inherent to its technological activities. These findings, beyond specific examples, can be generalized to all situations described in Propositions 2 and 3, for any distribution of $\theta$, by establishing that each firm can adjust within boundaries its contract offer to the lab, without changing the equilibrium strategies and the related equilibrium expected payoffs to all parties, in order to guarantee for itself a constant payoff whenever possible. This adjusted transfer function, $t_{i}^{o}$, is characterized by the following lemma.

Lemma 1 Suppose that $(\tilde{\mathbf{t}}, \tilde{\mathbf{x}}, \tilde{\mathbf{y}}, \tilde{\mathbf{z}})$ is a TSPNE. Define, for each $\theta$, the lower bound of firm i's gross payoff over all possible efficient choices ${ }^{39}$

$$
\underline{g}(\theta)=\inf _{\mathbf{x} \in X^{*}}\left[\tilde{g}_{i}(\mathbf{x}, \theta)\right],
$$

and the function $h: \Theta \rightarrow \mathcal{R}$, whose value depends on the realized state only, with

$$
\begin{gathered}
E_{\theta}[h(\theta)]=0, \\
v_{i}(\tilde{\mathbf{x}}, \tilde{\mathbf{y}}, \tilde{\mathbf{z}}, \theta)-\underline{g}(\theta) \leq h(\theta) \leq v_{i}(\tilde{\mathbf{x}}, \tilde{\mathbf{y}}, \tilde{\mathbf{z}}, \theta),
\end{gathered}
$$

all $\theta \in \Theta$. Then $t_{i}^{o}(\mathbf{x}, \theta)=\sup \left\{0, \tilde{g}_{i}(\mathbf{x}, \theta)-\left[v_{i}(\tilde{\mathbf{x}}, \tilde{\mathbf{y}}, \tilde{\mathbf{z}}, \theta)-h(\theta)\right]\right\}$ is a truthful best reply to $t_{j}$ that incentivizes the lab to choose an action which is undistinguishable from $\tilde{\mathbf{x}}$, in that it yields the same expected payoffs to all parties. However, for any realized $\theta$ the (ex-post) equilibrium payoffs are:

$$
v_{0}(\tilde{\mathbf{x}}, \theta)+h(\theta), \quad v_{i}(\tilde{\mathbf{x}}, \tilde{\mathbf{y}}, \tilde{\mathbf{z}}, \theta)-h(\theta), \quad v_{j}(\tilde{\mathbf{x}}, \tilde{\mathbf{y}}, \tilde{\mathbf{z}}, \theta),
$$

$i, j=1,2, j \neq i$.

The condition in (20) is needed for all profit expressions to remain unchanged in expectation, although the lab receives more than the expected level for some values of $\theta$, and less for others. In (21) the first inequality implies that contract offers to the lab are non-negative, and the second one that the equilibrium payoff to the firm is also non-negative in all states of nature. ${ }^{40}$

By introducing in their payment strategy an adjustment term $h(\theta)$, as defined in Lemma 1, a firm can condition its financial transfers on the realization of the technological risk, and thereby protect its ex-post payoff from adverse events. In unfavourable circumstances where uncertainties imply that

\footnotetext{
${ }^{39}$ Unless there exists a multiplicity of optima, $\underline{g}(\theta)$ does not differ from the equilibrium gross payoff $\tilde{g}_{i}(\tilde{\mathbf{x}}, \theta)$.

${ }^{40}$ The adjusted contract offer introduced in Lemma 1 remains compatible with an upfront part $\underline{t}_{i}^{o}$ paid to the agent. Supposing that $t_{i}^{o}(\tilde{\mathbf{x}}, \theta)>0$ for almost all $\theta$, the upfront payment is bounded from above by the non-negative threshold $\bar{T}_{i}=\max _{T>0}\left\{T \mid \tilde{g}_{i}(\tilde{\mathbf{x}}, \theta)-v_{i}(\tilde{\mathbf{x}}, \tilde{\mathbf{y}}, \tilde{\mathbf{z}}, \theta) \geq T\right.$, for almost all $\left.\theta\right\}$. Indeed, as long as $\underline{t}_{i}^{o} \leq \bar{T}_{i}$, equilibrium contingent payments $k_{i}^{o}(\tilde{\mathbf{x}}, \theta)=t_{i}^{o}(\tilde{\mathbf{x}}, \theta)-\underline{t}_{i}^{o}$ verify $k_{i}^{o}(\tilde{\mathbf{x}}, \theta) \geq \tilde{g}_{i}(\tilde{\mathbf{x}}, \theta)-v_{i}(\tilde{\mathbf{x}}, \tilde{\mathbf{y}}, \tilde{\mathbf{z}}, \theta)-\bar{T}_{i} \geq 0$, for almost all $\theta$.
} 
the gross equilibrium profit can be so low as to be strictly lower than $v_{i}^{*}$ with positive probability, then each firm can adjust its payment to earn the lower bound $\underline{g}(\theta) \leq v_{i}^{*}$ if an adverse event occurs (e.g., the lab's efforts are unsuccessful, or they cost much more than expected), or a superior profit level $\bar{v} \geq v_{i}^{*}$ that does not depend on $\theta$ otherwise. In more favourable circumstances where uncertainty does not prevent the gross equilibrium profit to be higher than the expected net equilibrium value $v_{i}^{*}$ for almost all $\theta$, each firm can guarantee for itself the latter value as an ex-post payoff, which is thus independent of the realized state of nature.

Toward a more formal characterization of adjusted payment strategies, that is risk-proof contract offers, we define the set $\bar{\Theta}(\bar{v})$ such that $\underline{g}(\theta) \leq \bar{v}$, with $\bar{v} \geq v_{i}^{*}$. Then:

Proposition 4 Suppose that $(\tilde{\mathbf{t}}, \tilde{\mathbf{x}}, \tilde{\mathbf{y}}, \tilde{\mathbf{z}})$ is a TSPNE: $(i)$ If $g(\theta) \leq E_{\theta}\left[\tilde{g}_{i}(\tilde{\mathbf{x}}, \theta)-\tilde{t}_{i}(\tilde{\mathbf{x}}, \theta)\right]$ for a subset of $\Theta$ of strictly positive measure, firm $i$ can guarantee for itself an ex-post profit equal to the gross equilibrium value $\underline{g}(\theta)$ for all $\theta \in \bar{\Theta}(\bar{v})$, and to the higher constant ${ }^{41} \bar{v}$ for all $\theta \in \Theta \backslash \bar{\Theta}(\bar{v})$, by addressing to the lab the risk-proof contract offer

$$
t_{i}^{o}(\mathbf{x}, \theta)= \begin{cases}0 & \text { if } \theta \in \bar{\Theta}(\bar{v}), \\ \sup \left\{0, \tilde{g}_{i}(\mathbf{x}, \theta)-\bar{v}\right\} & \text { if } \theta \in \Theta \backslash \bar{\Theta}(\bar{v}) .\end{cases}
$$

(ii) Otherwise, firm i can guarantee for itself the expected net equilibrium value $v_{i}^{*}$ as an ex-post profit for almost all states of nature, by addressing to the lab the risk-proof contract offer

$$
t_{i}^{o}(\mathbf{x}, \theta)=\sup \left\{0, \tilde{g}_{i}(\mathbf{x}, \theta)-v_{i}^{*}\right\} \text {. }
$$

This proposition establishes that truthful contract offers are available to the firms that shield them (at least partly) from adverse technological events by transferring (some or all of ) the burden of risk to the upstream lab. This insurance property holds in particular when R\&D externalities are nonnegative ( $\epsilon \geq 0)$, implying from Proposition 2 that the equilibrium payoff to the lab, in expectation, is exactly zero. In that case, valuable projects at the industry level are vulnerable since an unfavorable draw necessarily yields, ex-post, a negative net return to the external lab. This vulnerability is made more acute when risk-proof strategies are used that pass on the downside effect of uncertainty through risk-proof contingent payments. (Of course such a negative outcome can generalize to situations where profits are positive, as in Proposition 3, and still sufficiently close to zero.) As a direct implication, risk-averse investors are deterred to finance projects on the supply side of the intermedi-

\footnotetext{
${ }^{41}$ This constant $\bar{v}$ can be easily characterized.
} 
ate market for technology, and "fewer projects being funded means a weaker industry pipeline, and fewer new drugs"(Cockburn and Lerner, 2009, p. 5).

Some safeguarding measures can be designed that aim at avoiding the abandonment of projects that contribute positively to the total industry profit, but are characterized by a high degree of technological uncertainty. Such measures, in light of our results and their connections to the empirical evidence, appear less relevant for clinical development than for early-stage research characterized by significant economies of scope and technological spillovers. The financial back-up of a partner university, as commonly observed for startups since the emergence of biotech engineering, can be interpreted as a relevant attempt to insure promising spin-offs from unfavorable events. Our formal analysis also points to the introduction of regulatory constraints on the adjustment of payments, by big pharma companies, on the realization of technological risks.

We now consider cases with increasing returns to R\&D.

Non-decreasing returns to $\mathbf{R} \& \mathbf{D}$. In this section, for $i=1,2$, we assume that

$$
\frac{\partial^{2} \hat{g}_{i}}{\partial s_{i}^{2}} \geq 0
$$

where $s_{i}=x_{i}+y_{i}$. We identify simple conditions for Propositions 2 and 3 to remain valid.

Proposition 5 Suppose that returns to RED are non-decreasing, as in (22). If $\partial^{2} \hat{g}_{i} / \partial x_{i} \partial x_{j}>0$ (i.e., $x_{i}$ and $x_{j}$ are complementary for firm $i$, with $i, j=1,2, j \neq i$ ), which implies that $d y_{i}^{*} / d x_{j}>0$, then Propositions 2 and 3 still hold. Otherwise, a sufficient condition is $d y_{i}^{*} / d x_{j}>-1$.

The interplay of contracted-out and internal R\&D levels is central to that result. From Proposition 1 we already know that, for each firm $i$, non-decreasing R\&D returns imply that internal and contracted-out R\&D are strategic complements: $d y_{i}^{*} / d x_{i} \geq 0$. Then, there are two cases: (1) If $x_{j}$ and $x_{i}$ are complements inside firm $i^{\prime}$ s gross payoff function, that is $\partial^{2} \hat{g}_{i} / \partial x_{i} \partial x_{j} \geq 0$, then $y_{i}^{*}$ is monotone increasing with the other firm's contracted-out R\&D level $x_{j}$, that is $d y_{i}^{*} / d x_{j} \geq 0$. In that case, the respective effects of $x_{i}, x_{j}, y_{i}$ and $y_{j}$ on firms' gross profits are all congruent and Propositions 2 and 3 remain valid with non-decreasing returns as well. (2) When $x_{i}$ and $x_{j}$ are substitutes, in that $\partial^{2} \hat{g}_{i} / \partial x_{i} \partial x_{j}<0$, then $y_{i}^{*}$ decreases with the other firm's contracted-out R\&D level $x_{j}$, that is $d y_{i}^{*} / d x_{j}<0$. Here more R\&D received from the lab reduces the competitor's internal R\&D level, so 
firm $i$ 's gross profit may be impacted negatively. In the most extreme circumstances, the latter effect could possibly result in $d \hat{g}_{i} / d x_{i}$ being negative when $\partial \hat{g}_{i} / \partial x_{j}$ and $\partial \hat{g}_{i} / \partial y_{j}$ are both positive. The latter property however does not occur when the substitution effect is limited, more specifically when $d y_{i}^{*} / d x_{j}>-1$.

The next (deterministic) example illustrates all cases predicted by Proposition 5. It shows that while external and internal R\&D tasks are complementary (a consequence of non-decreasing returns to R\&D from Proposition 1), the client firms fully appropriate industry profit (as in Proposition 2) or concede to the lab a positive share of it (as in Proposition 3).

Example $4 \square$ The external lab's $R \mathcal{E} D$ cost is $\hat{f}_{0}(\mathbf{x})=\gamma\left(x_{1}^{2}+x_{2}^{2}\right) / 2-\delta x_{1} x_{2}$, with $\delta \in[-\gamma, \gamma), \gamma>0$, and the firms' internal RED costs are $\hat{f}_{i}\left(y_{i}\right)=\kappa+y_{i}^{2}, i=1,2, \kappa>0$. The downstream marginal cost of production is $c>0$, the final-market inverse demands are $p_{i}(\mathbf{q})=\sup \left\{0, a_{i}(\mathbf{x}, \mathbf{y})-q_{i}-q_{j}\right\}, i, j=$ $1,2, j \neq i$, where $p_{i}$ is the price, $\left(q_{i}, q_{j}\right)$ are quantities, and the price intercept $a_{i}(\mathbf{x}, \mathbf{y})=\left(a+s_{i}+\beta s_{j}\right)$, which involves spillovers measured by the parameter $\beta \in[0,1]$ borrowed from d'Aspremont and Jacquemin (1988). Then $s_{i}=x_{i}+y_{i}$ can be interpreted as the sum of $R\left(x_{i}\right)$ and $D\left(y_{i}\right)$, as in Vonortas (1994), here toward a quality improvement (e.g., increased drug safety). Non-cooperative profit maximization in quantities leads to $q_{i}^{*}(\mathbf{x}, \mathbf{y})=\left[(a-c)+s_{i}(2-\beta)+s_{j}(2 \beta-1)\right] / 3$. We have $\partial^{2} \hat{g}_{i} / \partial s_{i}^{2}=2(2-\beta)^{2} / 9>0$, so condition (22) is satisfied for all parameter values (increasing RED returns). Then, whether Proposition 2 or 3 applies depends on $\delta$ and $\beta$ : (i) if $\delta \geq 0(<0)$ then condition (12) (resp. condition (16)) holds; (ii) if $\beta \geq 1 / 2(<1 / 2)$ then condition (13) (resp. condition (17)) holds, directly from $\partial \hat{g}_{i} / \partial x_{j}=\partial \hat{g}_{i} / \partial y_{j}=$ $(2 / 3)(2 \beta-1) q_{i}^{*}(\mathbf{x}, \mathbf{y})$. Moreover, $\partial^{2} \hat{g}_{i} / \partial x_{j} \partial x_{i}=(2 / 9)(2-\beta)(2 \beta-1) \geq 0$ only if $\beta \geq 1 / 2$, and we have $d y_{i}^{*} / d x_{j}=3(2 \beta-1)(\beta-2) /\left[\left(\beta^{2}-\beta+7\right)\left(\beta^{2}-3 \beta-1\right)\right]>-1$ for $\beta<1 / 2$. Therefore, in this example the non-negative RED externalities case of Proposition 2 applies if $\beta \geq 1 / 2$ and $\delta \geq 0$, and the negative externalities case of Proposition 3 applies if $\beta<1 / 2$ and $\delta<0$.

An important lesson of Propositions 2 and 3 is that the interplay of indirect (through the lab) and direct (inter-firm) technological externalities drives the additivity status of the value function $v$ in (9), which in the end determines the distibution of industry profits. This characterization applies in all situations where the two types of externalities have the same sign, as formalized by the easyto-use conditions (12-13) and (16-17). It applies also in "mixed" cases where indirect externalities are negative, while direct externalities are not, or vice versa. For an illustration, consider again the 
previous example by setting $\delta=\beta=0, \gamma>1$, and by assuming that the firms rely exclusivey on the exernal lab $\left(y_{1}=y_{2}=0\right)$. Here we have non-negative indirect but negative direct externalities $\left(\partial^{2} \hat{f}_{0} / \partial x_{1} \partial x_{2}=0\right.$ and $\partial \hat{g}_{i} / \partial x_{j}<0$ for all positive final-market quantities). Then, for $\gamma$ approaching 1 from above, one finds $v(\{1\})=v(\{2\})=(a-c)^{2} / 5$, and $\Lambda=(a-c)^{2} / 4$, a case of strict subadditivity, implying from (18) that equilibrium payoffs are $v_{1}^{*}=v_{2}^{*}=v_{0}^{*} / 3=(a-c)^{2} / 20$.

\section{Incentives for More Integration}

The distribution of industry profits can be modified by a shift to a more integrated structure that unifies the lab with one of the two firms, or both. The analysis of incentives to integrate vertically, after having characterized the equilibrium profit distribution in the decentralized common agency setting, is consistent with industry practice as the acquisition of an R\&D unit by a big pharma company often follows a period of collaboration (Folta, 1998; Danzon and Grabowski, 2012).

Supposing that the owners of the lab and the two firms can participate in the equity market in order to depart from the initial outsourcing equilibrium characterized in the previous section, we assume that (i) initially, each entity is owned by distinct sets of individuals (no one can simultaneously be a seller and a buyer); (ii) when the lab and only one firm integrate vertically, the unified entity can agree to supply R\&D to the other firm by bargaining with it over the sharing of industry profits; and (iii) transaction costs are nil.

Consider first the situation in which the lab and the two firms all participate in some form of integration on the intermediate R\&D market. This occurs if the lab acquires the two firms and controls them as subsidiaries, or if the two firms share the ownership of the lab and control it as a joint venture, with choices of internal $R \& D$ and final-market strategies remaining non cooperative (no collusion). In these two cases there is no gain in joint profits to be earned vis-à-vis equilibrium payoffs of the common agency structure. This is because the truthfulness of the firms' equilibrium payment strategies implies that the lab is offered two transfer schedules which exactly reflect the respective shapes of the firms' gross profit functions (that is, $\left.\tilde{g}_{i}(\mathbf{x}, \theta), i=1,2\right)$. The lab thereby internalizes both direct and indirect externalities, and thus is incentivized to supply R\&D outputs that maximize the joint profits of all participants. It follows that the net share of joint profits accruing to each buyer of another firm's equity cannot improve on the amount of net profits received in the common agency 
equilibrium. Forward integration (i.e., the two users become subsidiaries of the lab) would imply the payment of $v_{i}^{*}$ by the lab to the firms' owners. Backward integration (i.e., the lab becomes a joint venture) would require the total payment of $v_{0}^{*}$ by the two firms for the ownership of the upstream assets. The equality $v_{0}^{*}+v_{1}^{*}+v_{2}^{*}=\Lambda$ holds in all cases, so there is no incentive for the lab and the two firms to form a unique entity, unless further assumptions are introduced (e.g., cost or demand parameters become a function of the governance structure). More formally:

Proposition 6 In the initial outsourcing equilibrium, the firms' non-cooperative transfers and the lab's forprofit $R \mathcal{E} D$ operations result in a maximum expected industry profit: $\tilde{\mathbf{x}} \in \arg \max _{\mathbf{x} \in X} E_{\theta}\left[\tilde{g}_{1}(\mathbf{x})+\tilde{g}_{2}(\mathbf{x})\right.$ $\left.-f_{0}(\mathbf{x})\right]$. Therefore, unless the firms coordinate internal RED operations ( $\left.\mathbf{y}\right)$ or collude in final-market commercial decisions $(\mathbf{z})$, there is no incentive for the lab to acquire the two firms and control them as subsidiaries, nor for the firms to share the ownership of the lab and control it as a joint venture.

As for uncertainty, it can only reduce incentives for the two firms to acquire and control the lab jointly, since by doing so they internalize the part of risks that can be transferred via adjusted payments to the supply side of the R\&D market. The viewpoint of the lab's owners is different though, as by acquiring the two firms they alleviate the burden of risk they bear in the initial outsourcing situation. Nevertheless, Proposition 6 establishes that the integrated structure cannot generate the value that is needed to finance such possible risk dilution benefits. The integration of all market participants can be financed only if the firms neutralize downstream strategic interactions by coordinating internal R\&D activities or/and final-market strategies.

It remains to investigate all alternative forms of integration that can allow the owners of the lab, or of the two firms, to privately appropriate a larger share of the industry maximum $\Lambda$ than in the decentralized outsourcing initial situation. Toward an equilibrium industry structure in the equity market, we consider the following discrete set of possible arrangements: the horizontal integration of firms 1 and 2 for the joint procurement of external R\&D (internal R\&D and final-market choices remaining non cooperative), the vertical integration of the lab with firm 1 , or with firm 2 . We consider in turn the situations in which the value function $v$ (in (9)) is superadditive ( $\epsilon \geq 0$, as in Proposition 2), then strictly subadditive ( $\epsilon<0$, as in Proposition 3), depending on the interplay of indirect (through the lab) and direct (inter-firm) technological externalities. The two cases are illustrated respectively by Figures 1 and 2, which represent the space of possible partitions of the maximum industry profit $\Lambda$ as a 2-simplex, with full appropriation by the lab (i.e., $v_{0}=\Lambda$ ) at the top vertex, and by either of 
the two firms at the bottom vertices. More generally, the payoffs to the lab and each of the two firms are proportional to the distance of the allocation point to the edge opposite to their respective vertex.

Non-negative R\&D externalities - When indirect and direct R\&D externalities are both nonnegative, or in "mixed" situations with positive and negative externalities where the former dominate, so that $v$ is superadditive $(\epsilon \geq 0)$, from Proposition 2 the lab only breaks even in equilibrium of the common agency structure, that is its expected profit is $v_{0}^{*}=0$, and the two firms thus expect to appropriate the total industry profit, $v_{1}^{*}+v_{2}^{*}=\Lambda$ (where $v_{i}^{*} \geq \underline{v}_{i}, k=1,2$ ). As there exists a continuum of firm equilibrium expected payoffs, the exact distribution $\left(v_{1}^{*}, v_{2}^{*}\right)$ can only reflect circumstances outside of the initial model specifications. Hereafter we formalize such circumstances by the bargaining powers $\left(\phi_{1}, \phi_{2}\right)$ in $[0,1]^{2}$, with $\phi_{1}+\phi_{2}=1$. They verify

$$
v_{k}^{*}=\underline{v}_{k}+\phi_{k}(\Lambda-\underline{v})
$$

where $k=1,2$, and $\left(\underline{v}_{1}, \underline{v}_{2}\right)$ is the disagreement point, with $\underline{v}=\underline{v}_{1}+\underline{v}_{2}$, so that,

$$
\phi_{k}=\frac{v_{k}^{*}-\underline{v}_{k}}{\Lambda-\underline{v}}
$$

Although with non-negative externalities joint R\&D procurement cannot increase the firms' joint profit, a larger individual share can be earned by a firm if it deviates unilaterally from the outsourcing equilibrium to acquire the lab. By exclusively controlling the lab, the vertically integrated entity $\{0, i\}$ benefits from a stronger bargaining position. In case of disagreement its payoff becomes $v(\{i\}) \geq \underline{v}_{i}$, while its rival $j$ 's payoff remains at $\underline{v}_{j}$, the standalone value. The bargaining process, in case of vertical integration, thus determines an expected payoff to the unified entity equal to

$$
v_{0+i}^{\{0, i\}}=v(\{i\})+\phi_{i}\left(\Lambda-v(\{i\})-\underline{v}_{j}\right)>v_{i}^{*}
$$

and an expected payoff to the outsider equal to

$$
v_{j}^{\{0, i\}}=\underline{v}_{j}+\phi_{j}\left(\Lambda-v(\{i\})-\underline{v}_{j}\right)<v_{j}^{*},
$$

with the weights $\left(\phi_{i}, \phi_{j}\right)$ as defined in (24), $i, j=1,2, j \neq i .^{42}$

\footnotetext{
${ }^{42} \mathrm{An}$ equality sign replaces the strict inequality signs in (25) and (26) only if the exclusive control of the lab by firm $i$
} 


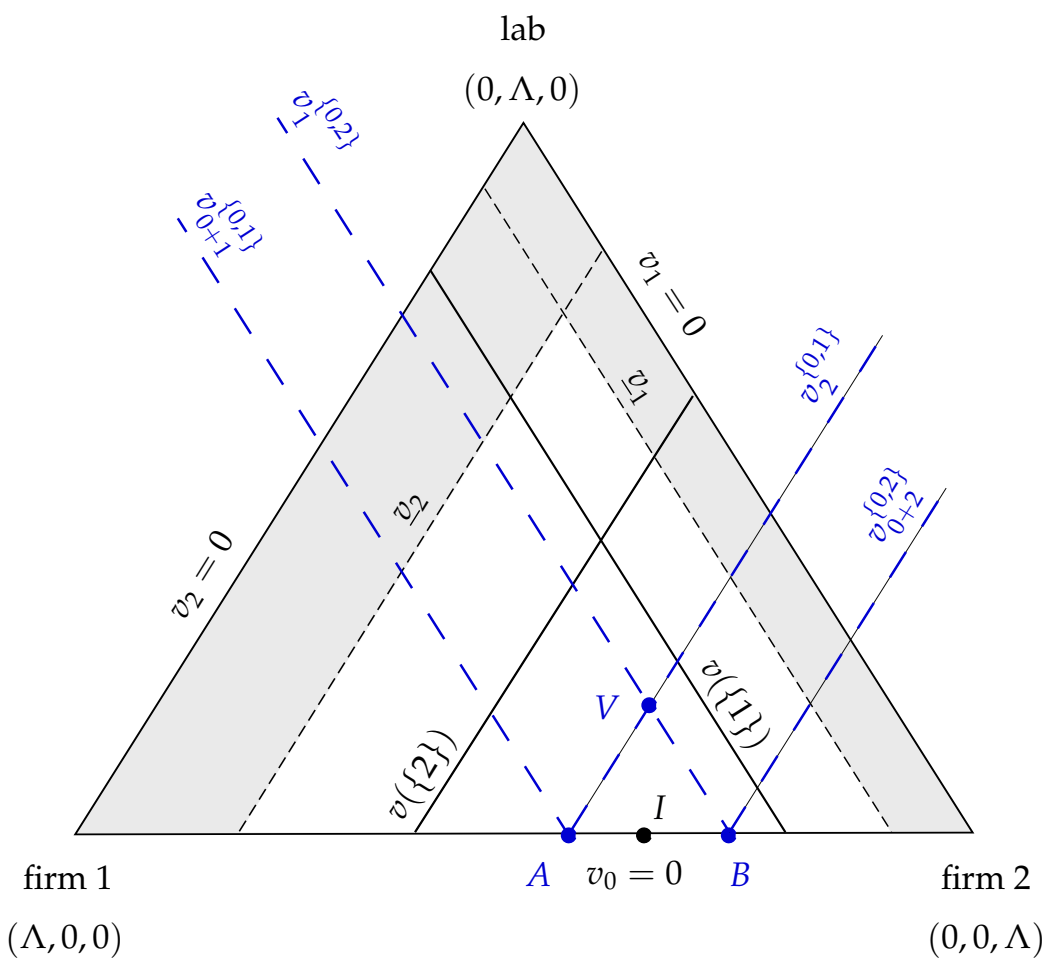

Figure 1: $v$ is superadditive $(\epsilon \geq 0)$, so the loci for $v(\{1\})$ and $v(\{2\})$ intersect inside the simplex. In the initial equilibrium (point $I$ ), in expectation the firms fully appropriate industry profits $\left(v_{1}^{*}+v_{2}^{*}=\Lambda\right.$ ), so the lab exactly breaks even $\left(v_{0}^{*}=0\right)$. Should a firm integrate the lab at no cost, it would appropriate more industry profits (point $A$ for firm 1, or $B$ for firm 2 ). However, competition in the equity market lowers firms' payoffs to $v_{i}^{\{0, j\}}$, for $i, j=1,2, j \neq i$, and results in the lab to appropriate a positive benefit, that is $v_{0}^{\{0,1\}}=v_{0}^{\{0,2\}}>0$ (point $V$ ).

As they face two competing alternatives, the lab's owners can choose the firm to integrate with. ${ }^{43}$ By selling out to firm $i$, they may expect to earn $v_{0}^{\{0, i\}}=v_{0+i}^{\{0, i\}}-v_{i}^{\{0, i\}}$, the difference between the expected payoff to the unified entity and the acquirers' residual claim. The two firms' respective owners thus compete in the equity market, and their willingness to pay is the difference between the profit they earn by acquiring the lab and the profit they earn should the lab integrate with the other firm. In comparison to the initial equilibrium situation, the firm that does not integrate, say firm $j$, is forced to concede what the other firm appropriates by acquiring the lab. Firm $i$ 's willingness to pay, entails no benefit vis-à-vis the standalone value, that is $v(\{i\})=\underline{v}_{i}$.

${ }^{43}$ For completeness, if the exclusive control of the lab strictly dominates the standalone option only for firm $i$, so that $v(\{i\})>\underline{v}_{i}$ and $v(\{j\})=\underline{v}_{j}$, then $v_{0+j}^{\{0, j\}}=v_{j}^{*}$. As there is no incentive for the latter firm $j$ to integrate vertically, in that case firm $i$ acquires the lab (it pays $\varepsilon>v_{0}^{*}=v_{0}^{\{1,2\}}=0$ with $\varepsilon$ arbitrarily small), and the equilibrium structure is $\{0, i\}$, with payoffs as in (25-26). If $v(\{i\})=\underline{v}_{i}$ as well, then no firm is interested in acquiring the lab. 
as an acquirer, is thus the sum of what it appropriates, and what the other would have appropriated, that is

$$
v_{0+i}^{\{0, i\}}-v_{i}^{\{0, j\}}=\phi_{j}\left(v(\{i\})-\underline{v}_{i}\right)+\phi_{i}\left(v(\{j\})-\underline{v}_{j}\right)>0,
$$

$i, j=1,2, j \neq i{ }^{44}$ Therefore, although firms are asymmetric, and appropriate different amounts by acquiring the lab, the willingness to pay is the same across the two firms. Competition in the equity market is thus frontal, and it leads to the integration of the lab with any of the two firms, indifferently.

In the equilibrium industry structure, firm $i$ 's owners have bidden their willingness to pay so they receive only $v_{i}^{\{0, j\}}<v_{i}^{*}$, a case of winner's curse. Uncertainty does not help, as by integrating the lab the acquirer cannot benefit any more from the contractual ability to shield (at least partly) its financial performance from adverse technological events. ${ }^{45}$ The rival $j$ 's owners are not better off, as by contracting out for $R \& D$ with the integrated entity $\{0, i\}$ they earn only $v_{j}^{\{0, i\}}<v_{j}^{*}$. As for the lab's owners, they earn a payoff equal to the common maximum bid, which from (24) and (27) is equal to

$$
v_{0}^{\{0,1\}}=v_{0}^{\{0,2\}}=\frac{v(\{1\})-\underline{v}_{1}}{\Lambda-\underline{v}}\left(v_{2}^{*}-\underline{v}_{2}\right)+\frac{v(\{2\})-\underline{v}_{2}}{\Lambda-\underline{v}}\left(v_{1}^{*}-\underline{v}_{1}\right)>v_{0}^{*}=0,
$$

with the strict inequality replaced by an equality sign only in the degenerate case where $v(\{i\})=\underline{v}_{i}$ for the two firms $(i=1,2)$. Otherwise, even if $v_{i}^{*}=\underline{v}_{i}$ for one of the two firms (say $i=1$ ), the inequality remains strict (as necessarily $\left.v_{2}^{*}-\underline{v}_{2}=\Lambda-\underline{v}-\left(v_{1}^{*}-\underline{v}_{1}\right)>0\right){ }^{46}$

Therefore, in expectation, the lab's owners extract a positive share of industry profits in the equity market (point $V$ in Figure 1), unlike in the initial equilibrium of the R\&D market (Proposition 2). This theoretical result is consistent with the empirical evidence in Higgins and Rodriguez (2006), where firms overbid for an external unit, and the acquirer succumbs to the winner's curse. More recently, Pisano (2015) also reflected on whether "pharmaceutical companies [are] paying more for R\&D by acquiring companies than by carrying out the R\&D themselves". According to our result, pharmaceutical companies pay more for R\&D by acquiring the lab than by engaging in a contractual

\footnotetext{
${ }^{44}$ In (27) we have $v_{0+i}^{\{0, i\}}-v_{i}^{\{0, j\}}=0$ only if $v(\{i\})-\underline{v}_{i}=v(\{j\})-\underline{v}_{j}=0$, or $\phi_{i}=v(\{j\})-\underline{v}_{j}=0, i, j=1,2, j \neq i$.

${ }^{45}$ However, integration is more attractive if it entails a reduced ability for firm $j$, as an outsider, to transfer risks to the lab when the latter is controlled by its rival $i$. Still, by integrating the lab the acquirer must bear the consequence of risks that were transferred upstream in the initial outsourcing situation. So even when vertical integration modifies the design of contracts and a redistribution of risks over R\&D market participants, at this level of generality it is not clear whether the net effect of that change mitigates the winner's curse problem or not.

${ }^{46}$ Otherwise, even if $v_{i}^{*}=\underline{v}_{i}$ for one of the two firms (say $i=1$ ), the inequality in (28) remains strict (as necessarily $\left.v_{2}^{*}-\underline{v}_{2}=\Lambda-\underline{v}-\left(v_{1}^{*}-\underline{v}_{1}\right)>0\right)$.
} 
arrangement with it. This is because, in this model, competition is tougher in the equity market, where only one firm can acquire the lab, and the opportunity cost of not being that firm is very high, whereas in the R\&D market firms can partially reconcile their antagonism through finely tuned contract offers.

Proposition 7 With non-negative RED externalities ( $\epsilon \geq 0$ ), one of the two firms - which are possibly asymmetric - acquires the lab with equal probability $1 / 2$. The bidding contest in the equity market implies a strictly higher expected profit to the lab, and a lower one to each firm, than in the initial outsourcing equilibrium.

The latter proposition points to exit payoffs to capital holders as a long-term financial incentive that can motivate investments in early-stage research activities (discovery). Indeed, from (28) the expected profit to the lab's owners in the equity market can be as high as the net industry level $\Lambda-v$, which occurs when $v(\{i\})$ is set equal to $\Lambda-\underline{v}_{j}, i, j=1,2, j \neq i$. This case describes rather extreme situations where, in the initial outsourcing equilibrium, the net marginal contribution of each firm to the total industry value is equal to zero, that is $v(\{1,2\})-v(\{i\})-\underline{v}_{j}=0$, for $i, j=1,2, j \neq i$, implying that having two firms instead of only one does not change the industry value.

On the other hand, exit payoffs can also be very low. Again from (28), the gain to the lab's owners in the equity market are negligible whenever the difference $v(\{i\})-\underline{v}_{i}$, for $i=1,2$, approaches zero. This situation relates typically to interesting situations where the lab's superior efficiency originates from economies of scope that can hardly be exploited when a firm controls the external unit exclusively for itself. ${ }^{47}$ In that case, the lab's owners, who exactly break-even in the initial outsourcing equilibrium (Proposition 2), cannot reapproriate industry profits by selling the equity to a firm. They thus have little incentive to invest in the first place, even when large economies of scope imply a project of high total value $\Lambda$.

Negative R\&D externalities - When indirect and direct R\&D externalities are both negative, or in "mixed" situations with positive and negative externalities where the latter dominate, so that $v$ is strictly subadditive $(\epsilon<0)$, from Proposition 3 the equilibrium expected payoffs in the initial outsourcing situation are $v_{0}^{*}=|\epsilon|>0$, and $v_{i}^{*}=v(\{i\})-|\epsilon| \geq \underline{v}_{i}, i=1,2$, so that $v_{1}^{*}+v_{2}^{*}<\Lambda$. As

\footnotetext{
${ }^{47}$ To illustrate, consider Example 4 above (see Section 4 ), with $\beta=1 / 2$ (so direct externalities are nil, for simplicity), and $\delta>0$ (economies of scope), so $\epsilon>0$. Then, whenever the cost parameter $\gamma$ takes a sufficiently high value, supposing that firm $i$ controls the lab for itself it must focus on internal R\&D (the $y_{i}$ dimension) to earn a maximum profit $v(\{i\})$ whic is only slightly higher than the standalone value $\underline{v}_{i}$.
} 
an alternative to the initial outsourcing situation, the firms can opt for an horizontal arrangement in order to procure jointly external $R \& D$. In that case they behave cooperatively as a unique principal on the intermediate market for technology, and fully appropriate the maximum industry profit, with the lab breaking even exactly in expectation (point $H$ in Figure 2 below). ${ }^{48}$ We thus have $v_{0}^{\{1,2\}}=0$ and $v_{1}^{\{1,2\}}+v_{2}^{\{1,2\}}=\Lambda$ (here the superscript $\{1,2\}$ refers to the industry structure with firms 1 and 2 procuring jointly).

The initial outsourcing equilibrium payoffs determine the firms' disagreement point $\left(v_{1}^{*}, v_{2}^{*}\right)$ when they bargain over the agent's expected payoff $v_{0}^{*}=\Lambda-v_{1}^{*}-v_{2}^{*}$. The firms' expected payoffs $\left(v_{1}^{\{1,2\}}, v_{2}^{\{1,2\}}\right)$ then verify

$$
v_{k}^{\{1,2\}}=v_{k}^{*}+\omega_{k}\left(\Lambda-v_{1}^{*}-v_{2}^{*}\right)
$$

where $k=1,2$, implying that bargaining powers $\left(\omega_{1}, \omega_{2}\right)$ in $[0,1]^{2}$, with $\omega_{1}+\omega_{2}=1$, are

$$
\omega_{k}=\frac{v_{k}^{\{1,2\}}-v_{k}^{*}}{\Lambda-v_{1}^{*}-v_{2}^{*}} .
$$

From Proposition 3 we know that $v_{0}^{*}=\Lambda-v_{1}^{*}-v_{2}^{*}>0$, implying that in (29) we have $v_{k}^{\{1,2\}} \geq v_{k}^{*}$ for $k=1,2$, with a strict inequality sign for at least one firm, implying that the latter firm earns a positive gain by shifting to the horizontal arrangement. Moreover, the definition of $\epsilon$ in (10) together with $v_{i}^{*}=v(\{i\})-|\epsilon|$ in (18) imply that $v_{j}^{*}=\Lambda-v(\{i\})$, so that $v_{j}^{\{1,2\}} \geq v_{j}^{*}$ and $v_{j}^{\{1,2\}}=\Lambda-v_{i}^{\{1,2\}}$ lead to $v_{i}^{\{1,2\}} \leq v(\{i\}), i, j=1,2, j \neq i$, again with a strict inequality sign for at least one firm. It follows that

$$
v(\{k\})-|\epsilon| \leq v_{k}^{\{1,2\}} \leq v(\{k\})
$$

where $k=1,2$, with at least one strict inequality sign. In (31) the first inequality states that any situation resulting in lower individual payoffs than in the initial equilibrium is rejected. The second inequality indicates that each firm's expected payoff in the horizontal arrangement is bounded from above by $v(\{i\})$, the value generated when it acquires the external lab without contracting with its rival.

\footnotetext{
${ }^{48} \mathrm{~A}$ horizontal arrangement here relates to the intermediate market for technology, as opposed to the final market for products, where the firms are assumed to remain competitors. This situation is similar to the cases observed by Majewski (2004) where firms engaged in a technology alliance jointly choose to outsource their R\&D to a third party in order to split costs.
} 


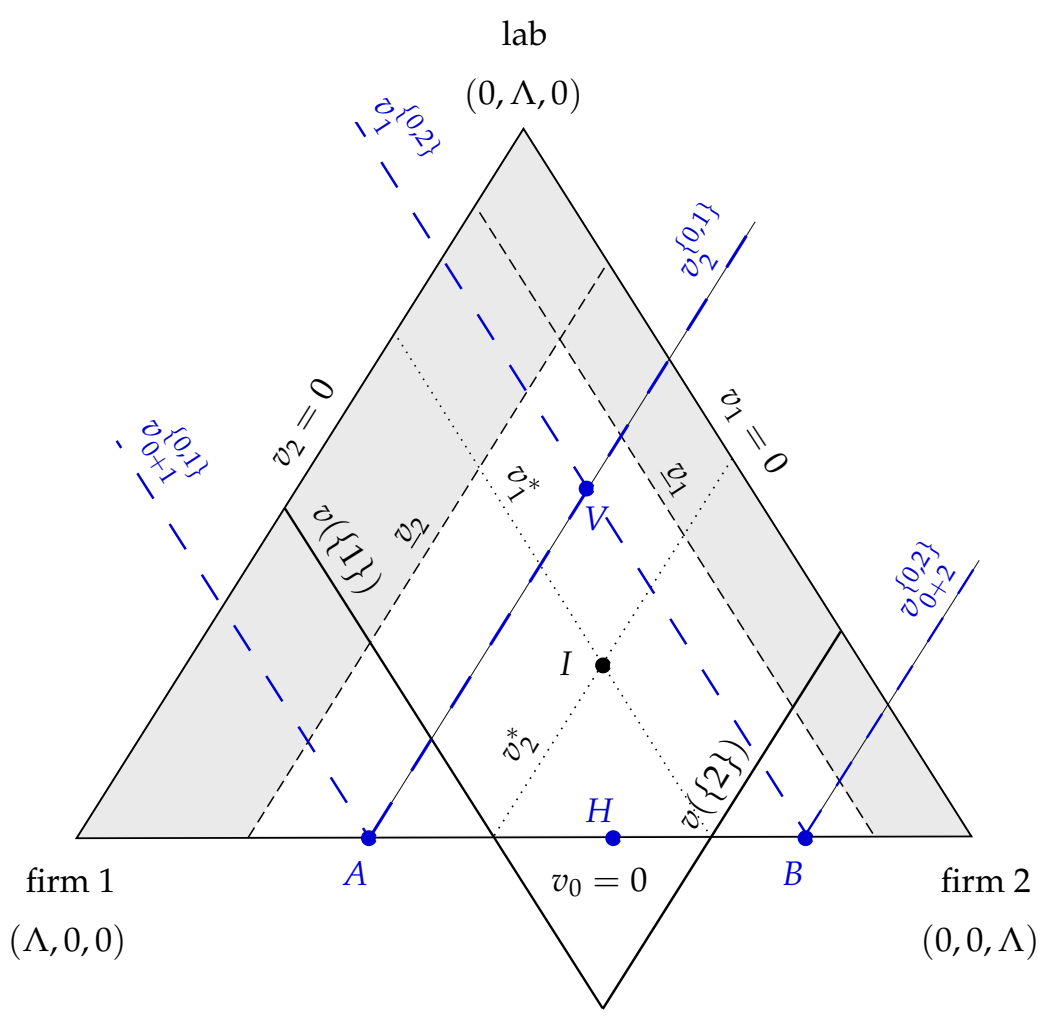

Figure 2: $v$ is strictly subadditive $(\epsilon<0)$, so the loci for $v(\{1\})$ and $v(\{2\})$ intersect outside the simplex. In the initial R\&D equilibrium (point $I$ ) the firms earn an expected profit $v_{i}^{*}=v(\{i\})-|\epsilon|, i=1,2$, and the lab earns $v_{0}^{*}>0$. By agreeing horizontally to coordinate R\&D outsourcing, the firms fully reappropriate industry profits (point $H$ ). Should a firm integrate vertically with the lab, at no cost, it would increase profits (point $A$ for firm 1, or $B$ for firm 2). The bidding contest to acquire the lab leads both firms to earn $v_{i}^{\{0, j\}}, i, j=1,2, j \neq i$ (point $V$ ) in the equilibrium industry structure.

While the two firms' joint profit is maximized in the horizontal arrangement, each firm has an incentive to depart unilaterally from $\{1,2\}$ by acquiring the lab, for a strictly ${ }^{49}$ higher disagreement payoff $v(\{i\})>v_{i}^{*}$ accruing to the integrated entity $\{0, i\}$, and a (weakly) lower disagreement payoff $\underline{v}_{j} \leq v_{j}^{*}$ to the other firm. By controlling the lab and benefitting exclusively from its technology, in case of disagreement the integrated entity can guarantee for itself the upper bound of the horizontal arrangement payoff in (31), while the outsider earns only its standalone value. The bargaining process, with vertical integration, implies an expected payoff to the unified entity equal to

$$
v_{0+i}^{\{0, i\}}=v(\{i\})+\omega_{i}\left(\Lambda-v(\{i\})-\underline{v}_{j}\right)>v_{i}^{\{1,2\}},
$$

\footnotetext{
${ }^{49}$ In this negative externalities situation $(\epsilon=\Lambda-v(\{i\})-v(\{j\})<0)$ we have $v(\{i\})>\Lambda-v(\{j\})=v_{i}^{*}$.
} 
and an expected payoff to the outsider equal to

$$
v_{j}^{\{0, i\}}=\underline{v}_{j}+\omega_{j}\left(\Lambda-v(\{i\})-\underline{v}_{j}\right)<v_{j}^{\{1,2\}},
$$

$i, j=1,2, j \neq i .^{50}$

The lab's owners, as in the non-negative externalities situation of the previous section, can thus make the two firms compete in the equity market by soliciting bids in order to appropriate a share of industry profits. Provided that no restriction is introduced that limits payment offers, again the firms have the same willingness to pay for the lab, that is

$$
v_{0+i}^{\{0, i\}}-v_{i}^{\{0, j\}}=\omega_{j}\left(v(\{i\})-\underline{v}_{i}\right)+\omega_{i}\left(v(\{j\})-\underline{v}_{j}\right)>0
$$

$i, j=1,2, j \neq i$, and any of them becomes the acquirer with the same probability $1 / 2$. The payoffs structure already obtained in the non-negative externalities case thus prevails, with each firm's respective owners earning exactly their outside value, $v_{1}^{\{0,2\}}<v_{1}^{\{1,2\}}$ and $v_{2}^{\{0,1\}}<v_{2}^{\{1,2\}}$, in any of the two possible equilibrium industry structures.

However, with negative externalities it is not a priori established that the lab's owners are better off post integration than in the initial outsourcing equilibrium. Inserting the expression of the firms' respective bargaining powers in (34), and reorganizing terms (see Appendix A.10), we find that

$$
v_{0}^{\{0,1\}}=v_{0}^{\{0,2\}}=\frac{v(\{1\})-\underline{v}_{1}}{|\epsilon|}\left(v_{2}^{\{1,2\}}-v_{2}^{*}\right)+\frac{v(\{2\})-\underline{v}_{2}}{|\epsilon|}\left(v_{1}^{\{1,2\}}-v_{1}^{*}\right) \geq v_{0}^{*}=|\epsilon|
$$

The value that can be extracted by the lab's owners by selling their property rights to any of the two - possibly asymmetric - firms (from $I$ to $V$ in Figure 2) is only weakly greater than the share of expected industry profits obtained in Proposition 3. To summarize:

Proposition 8 With negative RED externalities $(\epsilon<0)$ : (a) If the firms can commit not to integrate the lab they horizontally coordinate external technology sourcing and appropriate the total industry value $\Lambda$; $(b)$ Otherwise, one of the two firms acquires the lab with the same probability $1 / 2$, and the positive profit earned by the lab's owners in the equity market is only weakly superior than in the initial outsourcing equilibrium.

\footnotetext{
${ }^{50}$ The strict inequality sign in (33) is a consequence of $v(\{i\})>v_{i}^{*} \geq \underline{v}_{i}$, and possibly of firm $j$ 's strictly lower disagreement value $\underline{v}_{j}<v_{j}^{*}$ (this differs from the non-negative externalities case in (26)). Then the strict inequality in (32) follows from $v_{i}^{\{1,2\}}+v_{j}^{\{1,2\}}=v_{0+i}^{\{0, i\}}+v_{j}^{\{0, i\}}=\Lambda$, which leads to $v_{0+i}^{\{0, i\}}-v_{i}^{\{1,2\}}=v_{j}^{\{1,2\}}-v_{j}^{\{0, i\}}>0$.
} 
Part (a) of Proposition 8 focuses on cases where the firms, for some exogenous reasons (e.g., a regulation, or a strategic orientation), rule out the possibility to acquire the lab. When technological externalities are negative $(\epsilon<0)$, because multi-client R\&D operations entail diseconomies of scope and inter-firm knowledge spillovers are limited, or as a consequence of a strong business-stealing effect in the final market, the firms are more likely to coordinate horizontally the sourcing of new technology from the same external lab than in the case of positive externalities. This observation is reminicent of Majewski (2004), where evidence is found that "when collaborative agreements involve firms that compete in downstream markets, they tend to outsource their collaborative R\&D to a third party" (p. 2). Part (b) of Proposition 8 emphasizes that, with negative technological externalities (late-stage clinical development), the financial performance of the lab can only be weakly augmented in the equity market in comparison to the initial outsourcing equilibrium. As the upstream expected financial returns are already positive in the latter equilibrium, and monotone increasing with the magnitude of $\epsilon$ (in Proposition 3), the lab's owners face relatively weak incentives to sell ownership to a downstream firm when their activities are characterized by strongly negative externalities.

Financial constraints - Propositions 7 and 8 are derived under the assumption that bids are unrestricted. However, in real-world business circumstances a financial constraint might be introduced that limits firms' ability to compete for the control of an external entity. In the theoretical context of the model, the effect of such a constraint can be investigated by assuming that the price paid to the lab's owners, in case of vertical integration, cannot be so high as to imply a lower expected payoff to the acquiring firm than in the initial outsourcing equilibrium. Formally, the two firms' respective financial constraints are thus

$$
v_{i}^{*} \leq v_{i}^{\{0, i\}}=\Lambda-v_{j}^{\{0, i\}}-v_{0}^{\{0, i\}},
$$

$i, j=1,2, j \neq i$. In principle such constraints make it more difficult for a firm to acquire the lab when the latter makes positive profits than when it exactly breaks even. They also help identifying the profile of the most agressive bidder.

With non-negative R\&D externalities $(\epsilon \geq 0)$, as $\Lambda-v_{i}^{*}=v_{j}^{*}$ from Proposition 2, firm $i$ 's constraint in (36) becomes $v_{0}^{\{0, i\}} \leq v_{j}^{*}-v_{j}^{\{0, i\}}$. In other words, firm $i$ 's maximum bid, when it acquires the lab, must not exceed what firm $j$ has lost, as an outsider, because of the change in bargaining positions vis-à-vis the outsourcing situation. By using (23) and (26), firm $i$ 's financial constraint can thus be rewritten $v_{0}^{\{0, i\}} \leq \phi_{j}\left(v(\{i\})-\underline{v}_{i}\right), i, j=1,2, j \neq i$. The lab's owners face two competing bids, 
and select the highest, say the one of firm $i$ if $\phi_{j}\left(v(\{i\})-\underline{v}_{i}\right) \geq \phi_{i}\left(v(\{j\})-\underline{v}_{j}\right)$ which, by using (24), is equivalent to

$$
\frac{v_{i}^{*}-\underline{v}_{i}}{v(\{i\})-\underline{v}_{i}} \leq \frac{v_{j}^{*}-\underline{v}_{j}}{v(\{j\})-\underline{v}_{j}} .
$$

The comparison in (37) predicts that the acquirer is the "weaker" firm, that is the one whose net equilibrium expected payoff in the initial outsourcing situation (that is $v_{i}^{*}-\underline{v}_{i}$ ), relatively to the net profit that the integrated entity can guarantee for itself $\left(v(\{i\})-\underline{v}_{i}\right)$, is lower.

With negative R\&D externalities $(\epsilon<0)$, the same reasoning starting from (36) leads to the following condition for firm $i$ to be the one that acquires the lab:

$$
\frac{v_{i}^{\{1,2\}}-\underline{v}_{i}}{v(\{i\})-|\epsilon|-\underline{v}_{i}} \leq \frac{v_{j}^{\{1,2\}}-\underline{v}_{j}}{v(\{j\})-|\epsilon|-\underline{v}_{j}}
$$

Here the acquirer is the firm whose gain in the horizontal arrangement (that is $v_{i}^{\{1,2\}}-\underline{v}_{i}$ ), relatively to the initial equilibrium net expected payoff $\left(v_{i}^{*}-\underline{v}_{i}=v(\{i\})-|\epsilon|-\underline{v}_{i}\right)$, is lower.

The theoretical prediction of conditions $(37$ - 38) is compatible with the empirical evidence. In Higgins and Rodriguez (2006), the firms experiencing a deterioration of their research pipeline are found to be more likely to engage in the acquisition of a biotech entity. In Danzon et al. (2007), the financially strong firms appear to be less likely to engage in acquisitions. In other words, the most active firms in the equity market are not the ones with the highest profit prospects nor the deepest pockets.

\section{Conclusion}

Our formal analysis offers a new rationale for the low average profitability of the science-based businesses of biotech (Pisano, 2006a, 2010), and the high amount of risk they take in comparison to their pharma sponsors (Golec and Vernon, 2009; Thakor et al., 2017), as observed since the emergence of genetic engineering in the 1970s. In light of our results, and their connection with empirical observations, we believe that limited financial returns should not be seen as evidence of disappointing technological progress, but can be interpreted as a confirmation that economies of scope and inter-firm knowledge spillovers have been significant in the biotechnology domain. Indeed, in such circum- 
stances, our results establish that the decision by competing firms to outsource early-stage research activities to a common external unit leads most expected value, possibly very substantial, to be appropriated by downstream sponsors. This conclusion does not extend, in our theoretical framework, to contracted-out development services that are characterized by negative technological externalities. Moreover, the higher level of idiosyncratic risk (specific to an individual unit or project) observed in biotech firms than in pharma companies is explained in our model by the ability of downstream firms to transfer - at least partly - the financial consequences of R\&D uncertainties to the supply side of the market for technology by the means of contingent transfer payments.

The contracting-out of discovery and clinical tasks is attractive from the viewpoint of big pharma companies, because it allows them to transfer risks to an external unit whose distinctive capabilites, and the ability to internalize technological externalities, are sources of efficiency gains. However, the possible delinkage of upstream investment incentives from total industry value, and the vulnerability of investors' net returns to negative shocks, together theorize the abandonment of projects precisely in those early-stage areas that can generate critical advances toward new treatments or preventives. An important consequence is that, although the long-run perspective of substantial exit payoffs in the equity market can in some cases generate incentives to start a biotech unit, externalizing research does not always qualify as a relevant pathway to address the declining productivity in innovation that has characterized the biopharmaceutical sector over several decades. Our theoretical results thus do not support the view of R\&D outsourcing as the panacea often described by business experts or industry publications.

\section{References}

Allain, M-L., Henry, E. and Kyle, M.K., 2015, "Competition and the efficiency of markets for technology," Management Science, 62(4), pp. 1000-1019.

Amir, R., Evstigneev, I. and Wooders, J., 2003, "Noncooperative versus cooperative R\&D with endogenous spillover rates," Games and Economic Behavior, 42(2), 183-207.

Argyres, N.S. and Liebeskind, J.P., 2002, "Governance inseparability and the evolution of US biotechnology industry," Journal of Economic Behavior and Organization, 47(2), 197-219.

Arora A., Fosfuri, A. and Gambardella, A., 2001, "Markets for technology and their implications for corporate strategy," Industrial and Corporate Change, 10(2), 419-451.

Arora, A. and Gambardella, A., 2010, "Ideas for rent: an overview of markets for technology " Industrial and Corporate Change, 19(3), 775-803. 
Arora, A., Gambardella, A., Pammolli, F. and Riccaboni, M., 2004a, "The nature and the extent of the market for technology in biopharmaceuticals," in: Cesaroni, F., Gambardella, A. and Garcia-Fontes, W., editors, RED, Innovation and Competitiveness in the European Chemical Industry, Ch. 7, Springer, 175-202.

Arora, A., Vogt, W.B. and Yoon, J., 2004b, "Does in-house R\&D increase bargaining power? Evidence from the pharmaceutical industry". Available at SSRN: http://ssrn.com/abstract=670304 or http:/ /dx.doi.org/10.2139/ssrn.670304

Azoulay, P., 2004. Capturing knowledge within and across firm boundaries: Evidence from clinical development," American Economic Review, 94(5), 1591-1612.

Bali, H., de Lima, B. and Yang, C., 2013, CROs (Contract Research Organisations) and other outsourced pharmaceutical support services: MEA drivers and trends. Available at http:/ / resultshealthcare.com

Bernheim, B.D. and Whinston, M.D., 1986a, “Common agency,” Econometrica, 54(4), 923-942.

Bernheim, B.D. and Whinston, M.D., 1986b, “Menu auctions, resource allocation, and economic influence," Quarterly Journal of Economics, 101(1), 1-32.

Bernheim, B.D. and Whinston, M.D., 1998, "Exclusive dealing," Journal of Political Economy, 106(1), pp. 64-103.

Bernheim, B.D., Peleg, B. and Whinston, M.D., 1987, "Coalition-proof Nash equilibria: I Concepts," Journal of Economic Theory, 42(1), 1-12.

Bhattacharya, S. and Guriev, S., 2006, "Patents vs. trade secrets: Knowledge licensing and spillover," Journal of the European Economic Association, 4(6), 1112-1147.

Bhattacharya, S. and Guriev, S., 2013, "Control rights over intellectual property," Journal of Industrial Economics, 61(3), 564-591.

Billette de Villemeur, E. and Versaevel, B., 2003, "From private to public common agency," Journal of Economic Theory, 111(2), pp. 305-309.

Billette de Villemeur, E. and Versaevel, B., 2017, “One lab, two firms, many possibilities: on R\&D outsourcing in the biopharmaceutical industry," MPRA Paper, No76903.

Bloom, N., Schankerman, M. and Van Reenen, J., 2013, “Identifying technology spillovers and product market rivalry," Econometrica, 81(4), 1347-1393.

Ceccagnoli, M., Higgins, M. and Palermo, V., 2014, "Behind the scenes: sources of complementarity in R\&D," Journal of Economics and Management Strategy, 23(1), 125-148.

Cockburn, I.M. and Lerner, J, 2009, "The cost of capital for early- stage biotechnology ventures," Congressional Briefing. Available at: http://buchpedersen.com/wp-content/uploads/2016/02/TheCostof-Capital-for-Early-Stage-Biotechnology-Ventures ${ }_{C}$ ockburnLerner.pdf

Cockburn, I.M. and Henderson, R.M., 2001, "Scale and scope in drug development: Unpacking the advantages of size in pharmaceutical research," Journal of Health Economics, 20(6), 1033-57.

Cohen, W. M., Nelson, R.R. and Walsh, J.P., 2000, "Protecting their intellectual assets: Appropriability conditions and why US manufacturing firms patent (or not)," National Bureau of Economic Research.

Comanor, W. and Scherer, F.M., 2013, "Mergers and innovation in the pharmaceutical industry," Journal of Health Economics, 32(1), 106-113. 
d'Aspremont, C. and Jacquemin, A., 1988, "Cooperative and noncooperative R\&D in duopoly with spillovers," American Economic Review, 78(5), 1133-1137.

Danzon, P., Nicholson, S. and Sousa Pereira, N., 2005, "Productivity in pharmaceutical-biotechnology R\&D: the role of experience and alliances," Journal of Health Economics, 24(2), 317-339.

Danzon, P., Epstein, A. and Nicholson. S., 2007, "Mergers and acquisitions in the pharmaceutical and biotech industries," Managerial and Decision Economics, 28(4-5), 307-328.

Danzon, A. and Grabowski, H., 2012, "Mergers, acquisitions, and alliances," in: Danzon, P. and Nicholson, S., editors, The Economics of the Biopharmaceutical Industry, Ch. 18, Oxford University Press, 552-577.

DiMasi, J.A., Hansen, R.W., Grabowski, H.G., and Lasagna, L., 1991, “Cost of innovation in the pharmaceutical industry," Journal of Health Economics, 10(2),107-142.

DiMasi, J.A., Hansen, R.W., and Grabowski, H.G., 2003, “The price of innovation: new estimates of drug development costs," Journal of Health Economics, 22(2), 151-185.

DiMasi, J.A., Hansen, R.W., and Grabowski, H.G., 2016, "Innovation in the pharmaceutical industry: New estimates of R\&D costs," Journal of Health Economics, 47(C), 20-33.

Folta, T.B., 1998, "Governance and uncertainty: The trade-off between administrative control and commitment," Strategic Management Journal, 19(11), 1007-28.

Galambos, L. and Sturchio, J.L., 1998, "Pharmaceutical firms and the transition to biotechnology: A study in strategic innovation," Business History Review, 72(2), 250-278.

Getz, K.A., 2007, “CRO shifts in the outsourcing market,” Applied Clinical Trials, 16(5), 35-38.

Golec, J. and Vernon, J.A., 2009, "Financial risk of the biotech industry versus the pharmaceutical industry," Applied Economics and Health Policy, 7(3), 155-65.

Guedj, I., 2005, “Ownership vs. contract: how vertical integration affects investment decisions in pharmaceutical R\&D," McCombs Research Paper Series, No. FIN-01-06.

Hagedoorn, J. and Wang, N., 2012, "Is there complementarity or substitutability between internal and external R\&D strategies?," Research Policy, 41(6), 1072-1083.

Hagedoorn, J. and Hesen, G., 2007, "Contract law and the governance of inter-firm technology partnerships: An analysis of different modes of partnering and their contractual implications," Journal of Management Studies, 44(3), 342-366.

Henderson, R.M. and Cockburn, I.M., 1996, "Scale, scope and spillovers: the determinants of research productivity in drug discovery," RAND Journal of Economics 27(1), 32-59.

Hernandez-Villafuerte, K., Sussex, J., Robin, E., Guthrie, S. and Wooding, S., 2017, "Economies of scale and scope in publicly funded biomedical and health research: evidence from the literature," Health Research Policy and Systems 15(1), 1-17.

Henriques, I., 1990, "Cooperative and noncooperative R\&D in duopoly with spillovers: Comment," American Economic Review, 80(3), 638-640.

Higgins, M.J., 2007, "The allocation of control rights in pharmaceutical alliances," Journal of Corporate Finance, 13(1), 58-75.

Higgins, M.J. and Rodriguez, D., 2006, "The outsourcing of R\&D through acquisitions in the pharmaceutical industry," Journal of Financial Economics, 80(2), 351-383. 
Ho, S.J., 2009, “Information leakage in innovation outsourcing," RED Management, 39(5), 431-443. Hoos, A., 2016, "Development of immuno-oncology drugs - from CTLA4 to PD1 to the next generations," Nature Reviews Drug Discovery, 15, 235-247.

Keith, J.A., Bigger, L.A., Phyllis A.A., Maes, A.E. and Daems, R., 2013, “Delivering the promise of the Decade of Vaccines: Opportunities and challenges in the development of high quality new vaccines," Vaccines, 31(S), B184-B193.

Lai, E., Riezman, R. and Wang, P., 2009, “Outsourcing of innovation,” Economic Theory, 38(3), 485-515.

Laussel, D. and Le Breton, M., 2001, "Conflict and cooperation: The structure of equilibrium payoffs in common agency," Journal of Economic Theory, 100(1), 93-128.

Lerner, J. and Merges, R.P., 1998, "The control of technology alliances: An empirical analysis of the biotechnology industry," Journal of Industrial Economics, 46(2), 125-156.

Macher, J.T. and Boerner, Ch. S., 2006, "Experience and scale and scope Economies: Trade-offs and performance in development," Strategic Management Journal, 27(9), 845-865.

Majewski, S., 2004, "How do consortia organize collaborative R\&D?: Evidence from the national cooperative research act," Discussion Paper 483, Harvard Law School.

Martimort, D., 2007, “Multi-Contracting Mechanism Design,” in: Blundell, R., Newey W. and Person, T., editors, Advances in Economic Theory Proceedings of the World Congress of the Econometric Society, Cambridge University Press.

Mestre-Ferrandiz, J.M., Sussex, J. and Towse, A., 2012, The RED Cost of a New Medicine. London: Office of Health Economics.

Motta, M., 1992, "Cooperative R\&D and vertical product differentiation, International Journal of Industrial Organization, 10(4), 643-661.

Munos, B. 2009, "Lessons from 60 years of pharmaceutical innovation,"Nature Reviews Drug Discovery, 8, 959-968.

Pammolli, F., Magazzini, L. and Riccaboni, M., 2011, "The productivity crisis in pharmaceutical R\&D,"Nature Revue Drug Discovery, 10(6), 428-438.

Pisano, G.P., 1991, "The governance of innovation: vertical integration and collaborative arrangements in the biotechnology industry," Research Policy, 20(3), 237-249.

Pisano, G.P., 2006a, Science Business: The Promise, the Reality and the Future of Biotech, Harvard Business School Press.

Pisano, G.P., 2006b, "Can science be a business? Lessons from biotech," Harvard Business Review, 84(10), 114-124.

Pisano, G.P., 2010, “The evolution of science-based business: Innovating how we innovate," Industrial and Corporate Change, 19(2), 465-482.

Pisano, G.P., 2015, “Big pharma needs to get busy in the lab: Blanket generalizations about biotech firms being more efficient are unfounded," The Wall Street Journal, March 19. Available at: www.wsj.com/ articles/gary-pisano-big-pharma-needs-to-get-busy-in-the-lab-1426805547.

Robinson, D.T. and Stuart, T.E., 2000, "The allocation of control in biotechnology strategic alliances," Organization Science Winter Conference. Available at: https:/ /faculty.fuqua.duke.edu/oswc/2000/papers / thursday/TobyStuart.pdf 
Robinson, D.T. and Stuart, T.E., 2007, “Financial contracting in biotech strategic alliances," Journal of Law and Economics, 50(3), 559-595.

Rothaermel, F.T., 2001, "Incumbent's advantage through exploiting complementary assets via interfirm cooperation," Strategic Management Journal, 22(6-7), 687-699.

Rydzewski, R.M., 2008, Real World Drug Discovery: A Chemist's Guide to Biotech and Pharmaceutical Research, Amsterdam: Elsevier.

Sancheti, S., Thomas, H., van den Heuvel, R. and Verhaeghe, A.J, 2018, RED 2030: Reinvent innovation and become an RED front-runner by 2030, KPMG International. Available at: https:/ /assets.kpmg.com/ content/dam/kpmg/xx/pdf/2018/11/r-and-d-2030-thriving-on-disruption-series.pdf

Scannell, J.W., Blanckley, A., Boldon, H. and Warrington, B., 2012, “Diagnosing the decline in pharmaceutical R\&D efficiency", Nature Reviews Drug Discovery, 11, 191-200.

Scherer, F.M., 2001, “The link between gross profitability and pharmaceutical R\&D spending," Health Affairs, 20(5), pp. 216-220.

Scherer, F.M., 2010, "Pharmaceutical innovation," in: Hall, B.H. and Rosenberg, N., editors, Handbook of The Economics of Innovation, Vol. 1, Ch. 12, pp. 539-574.

Scherer, F.M., 2011, "R\&D costs and productivity in biopharmaceuticals," Faculty Research Working Paper Series, Harvard Kennedy School, RWP11-046.

Schuhmacher, A., Gassmann, O. and Hinder, M., 2016, "Changing R\&D models in research-based pharmaceutical companies," Journal of Translational Medecine, 14(1), pp. 105-115.

Shuchman, M., 2007, "Commercializing Clinical Trials Risks and Benefits of the CRO Boom," New England Journal of Medicine, 357(14), 1365-1368.

Spulber, D.F., 2013, "How do competitive pressures affect incentives to innovate when there is a market for inventions?," Journal of Political Economy, 121(6), 1007-1054.

Stuart, T.E., Hoang H. and Ralph C.H., 1999, "Interorganizational endorsements and the performance of entrepreneurial ventures," Administrative Science Quarterly, 44(2), pp. 315-349.

Symeonidis, G., 2003, “Comparing Bertrand and Cournot equilibria in a differentiated duopoly with product R\&D," International Journal of Industrial Organization, 21(1), 39-55.

Tang, J., Shalabi, A. and Hubbard-Lucey, M., 2018, “Comprehensive analysis of the clinical immunooncology landscape," Annals of Oncology, 29, pp. 84-91.

Taylor, K., Shah, S. and Stockbridge, M., 2016, Balancing the RED Equation: Measuring the Return from Pharmaceutical Innovation, Deloitte Centre for Health Solutions. Available at: https:/ /www2.deloitte.com/ content/dam/Deloitte/uk/Documents/life-sciences-health-care/deloitte-uk-measuring-the-returnpharma-report-2016.pdf

Thakor, R. T., Anaya, N., Zhang, Y., Vilanilam, C., Siah, K. W., Wong, C. H., and Lo, A. W., 2017, "Just how good an investment is the biopharmaceutical sector?," Nature Biotechnology, 35(12), pp. 1149-1157.

Topkis, D., 1995, “Comparative statics of the firm," Journal of Economic Theory, 67(2), 370-401.

Vencatachellum, D. and Versaevel, B., 2009, "R\&D delegation in a duopoly with spillovers," The B.E. Journal of Economic Analysis E Policy, 9(1) (Contributions), Article 55. 
Vonortas, N.S., 1994, "Inter-firm cooperation with imperfectly appropriable research," International Journal of Industrial Organization, 12(3), 413-435.

Wilson, E., Wallach, M., and Willoughby, R., 2016, CRO Industry Primer, Credit Suisse. Available at: https:/ / research-doc.credit-suisse.com

\section{A Appendix}

We first develop the derivatives $\frac{d y_{j}^{*}}{d x_{j}}$ and $\frac{d y_{i}^{*}}{d x_{j}}$, which are needed to prove Propositions 1 to 5 afterwards.

\section{A.1 Derivation of $\frac{d y_{j}^{*}}{d x_{j}}$ and $\frac{d y_{i}^{*}}{d x_{j}}$}

As the arguments $x_{i}$ and $y_{i}$ enter additively into $g_{i}$ (hence $\hat{g}_{i}$ ), we have

$$
\frac{\partial \hat{g}_{i}\left(x_{i}+y_{i}^{*}, x_{j}, y_{j}^{*}\right)}{\partial x_{i}}-\frac{\partial \hat{f}_{i}\left(y_{i}^{*}\right)}{\partial y_{i}}=0,
$$

and similarly

$$
\frac{\partial \hat{g}_{j}\left(x_{j}+y_{j}^{*}, x_{i}, y_{i}^{*}\right)}{\partial x_{j}}-\frac{\partial \hat{f}_{j}\left(y_{j}^{*}\right)}{\partial y_{j}}=0,
$$

where the Nash strategies $y_{i}^{*}=y_{i}^{*}\left(x_{i}, x_{j}\right)$ and $y_{j}^{*}=y_{j}^{*}\left(x_{i}, x_{j}\right)$ result from the two firms' noncooperative profit-maximization in their respective internal $\mathrm{R} \& \mathrm{D}$ levels, for $i, j=1,2, j \neq i$.

Differentiating (39) and (40) w.r.t. $x_{j}$, and using again $s_{i}=x_{i}+y_{i}$ in $\hat{g}_{i}$, and $s_{j}=x_{j}+y_{j}$ in $\hat{g}_{j}$, we obtain the system of equations

$$
\left(\begin{array}{cc}
\frac{\partial^{2} \hat{g}_{i}}{\partial x_{i} \partial y_{j}} & \frac{\partial^{2} \hat{g}_{i}}{\partial x_{i}^{2}}-\frac{\partial^{2} \hat{f}_{i}}{\partial y_{i}^{2}} \\
\frac{\partial^{2} \hat{g}_{j}}{\partial x_{j}^{2}}-\frac{\partial^{2} \hat{f}_{j}}{\partial y_{j}^{2}} & \frac{\partial^{2} \hat{g}_{j}}{\partial x_{j} \partial y_{i}}
\end{array}\right)\left(\begin{array}{c}
\frac{d y_{j}^{*}}{d x_{j}} \\
\frac{d y_{i}^{*}}{d x_{j}}
\end{array}\right)=\left(\begin{array}{c}
-\frac{\partial^{2} \hat{g}_{i}}{\partial x_{i} \partial x_{j}} \\
-\frac{\partial^{2} \hat{g}_{j}}{\partial x_{j}^{2}}
\end{array}\right),
$$

where $\hat{g}_{i}=\hat{g}_{i}\left(x_{i}+y_{i}^{*}, x_{j}, y_{j}^{*}\right), \hat{g}_{j}=\hat{g}_{j}\left(x_{j}+y_{j}^{*}, x_{i}, y_{i}^{*}\right), \hat{f}_{i}=\hat{f}_{i}\left(y_{i}^{*}\right)$, and $\hat{f}_{j}=\hat{f}_{j}\left(y_{j}^{*}\right)$, for clarity.

This yields the solution

$$
\left(\begin{array}{c}
\frac{d y_{j}^{*}}{d x_{j}} \\
\frac{d y_{i}^{*}}{d x_{j}}
\end{array}\right)=\frac{1}{\Delta}\left(\begin{array}{cc}
-\frac{\partial^{2} \hat{g}_{j}}{\partial x_{j} \partial y_{i}} & \frac{\partial^{2} \hat{g}_{i}}{\partial x_{i}^{2}}-\frac{\partial^{2} \hat{f}_{i}}{\partial y_{i}^{2}} \\
\frac{\partial^{2} \hat{g}_{j}}{\partial x_{j}^{2}}-\frac{\partial^{2} \hat{f}_{j}}{\partial y_{j}^{2}} & -\frac{\partial^{2} \hat{g}_{i}}{\partial x_{i} \partial y_{j}}
\end{array}\right)\left(\begin{array}{c}
-\frac{\partial^{2} \hat{g}_{i}}{\partial x_{i} \partial x_{j}} \\
-\frac{\partial^{2} \hat{g}_{j}}{\partial x_{j}^{2}}
\end{array}\right)
$$

where

$$
\Delta=\left(\frac{\partial^{2} \hat{g}_{i}}{\partial x_{i}^{2}}-\frac{\partial^{2} \hat{f}_{i}}{\partial y_{i}^{2}}\right)\left(\frac{\partial^{2} \hat{g}_{j}}{\partial x_{j}^{2}}-\frac{\partial^{2} \hat{f}_{j}}{\partial y_{j}^{2}}\right)-\frac{\partial^{2} \hat{g}_{i}}{\partial x_{i} \partial y_{j}} \frac{\partial^{2} \hat{g}_{j}}{\partial x_{j} \partial y_{i}}
$$


We thus have

$$
\begin{aligned}
& \frac{d y_{j}^{*}}{d x_{j}}=\frac{1}{\Delta}\left[\frac{\partial^{2} \hat{g}_{j}}{\partial x_{j} \partial y_{i}} \frac{\partial^{2} \hat{g}_{i}}{\partial x_{i} \partial x_{j}}-\left(\frac{\partial^{2} \hat{g}_{i}}{\partial x_{i}^{2}}-\frac{\partial^{2} \hat{f}_{i}}{\partial y_{i}^{2}}\right) \frac{\partial^{2} \hat{g}_{j}}{\partial x_{j}^{2}}\right], \\
& \frac{d y_{i}^{*}}{d x_{j}}=\frac{1}{\Delta}\left[\frac{\partial^{2} \hat{g}_{i}}{\partial x_{i} \partial y_{j}} \frac{\partial^{2} \hat{g}_{j}}{\partial x_{j}^{2}}-\left(\frac{\partial^{2} \hat{g}_{j}}{\partial x_{j}^{2}}-\frac{\partial^{2} \hat{f}_{j}}{\partial y_{j}^{2}}\right) \frac{\partial^{2} \hat{g}_{i}}{\partial x_{i} \partial x_{j}}\right] .
\end{aligned}
$$

We know that $\frac{\partial^{2} \hat{f}_{i}\left(y_{i}^{*}\right)}{\partial y_{i}^{2}} \geq 0$ (by assumption) and $\frac{\partial^{2} \hat{g}_{i}}{\partial x_{i}^{2}}-\frac{\partial^{2} \hat{f}_{i}}{\partial y_{i}^{2}}<0$ (second-order condition), which holds also for firm $j$. As $\frac{\partial^{2} \hat{g}_{i}}{\partial x_{i} \partial y_{j}}$ and $\frac{\partial^{2} \hat{g}_{j}}{\partial x_{j} \partial y_{i}}$ have the same sign (by assumption), $\left\|\frac{\partial^{2} \hat{g}_{i}}{\partial x_{i}^{2}}\right\| \geq\left\|\frac{\partial^{2} \hat{g}_{i}}{\partial x_{i} \partial y_{j}}\right\|$ and $\left\|\frac{\partial^{2} \hat{g}_{j}}{\partial x_{j}^{2}}\right\| \geq\left\|\frac{\partial^{2} \hat{g}_{j}}{\partial x_{j} \partial y_{i}}\right\|\left(\right.$ see (7)), we obtain from (42) that $\Delta \geq 0 .{ }^{51}$

Moreover, we know also from Henriques (1990) that the reaction functions in the internal R\&D space $\left(y_{i}, y_{j}\right)$ cross "correctly", so that the Nash equilibrium $\left(y_{i}^{*}, y_{j}^{*}\right)$ is stable, only if

$$
\left|\frac{\partial^{2}\left[\hat{g}_{i}\left(x_{i}+y_{i}, x_{j}, y_{j}\right)-\hat{f}_{i}\left(y_{i}\right)\right]}{\partial y_{i}^{2}} / \frac{\partial^{2}\left[\hat{g}_{i}\left(x_{i}+y_{i}, x_{j}, y_{j}\right)-\hat{f}_{i}\left(y_{i}\right)\right]}{\partial y_{i} \partial y_{j}}\right|<1
$$

for $i, j=1,2, j \neq i$.

Again, the argument $s_{i}=x_{i}+y_{i}$ in $\hat{g}_{i}$, together with $\hat{f}_{i}$ being a function of $y_{i}$ only, imply that $\frac{\partial^{2}\left[\hat{g}_{i}\left(x_{i}+y_{i}, x_{j}, y_{j}\right)-\hat{f}_{i}\left(y_{i}\right)\right]}{\partial y_{i} \partial y_{j}}=\frac{\partial^{2} \hat{g}_{i}\left(x_{i}+y_{i}, x_{j}, y_{j}\right)}{\partial y_{i} \partial y_{j}}=\frac{\partial^{2} \hat{g}_{i}\left(x_{i}+y_{i}, x_{j}, y_{j}\right)}{\partial x_{i} \partial y_{j}}$, so that (45) becomes $\left|\left(\frac{\partial^{2} \hat{g}_{i}}{\partial x_{i}^{2}}-\frac{\partial^{2} \hat{f}_{i}}{\partial y_{i}^{2}}\right) / \frac{\partial^{2} \hat{g}_{i}}{\partial x_{i} \partial y_{j}}\right|<$ 1. The latter inequality, together with $\frac{\partial^{2} \hat{g}_{i}}{\partial x_{i}^{2}}-\frac{\partial^{2} \hat{f}_{i}}{\partial y_{i}^{2}}<0$ for $i=1,2$ (second-order condition) and $\frac{\partial^{2} \hat{g}_{i}}{\partial x_{i} \partial y_{j}} \frac{\partial^{2} \hat{g}_{j}}{\partial x_{j} \partial y_{i}} \geq 0$ for $i, j=1,2, j \neq i$ (partial cross-derivatives, for both firms, have the same sign by assumption) imply from (42) that $\Delta$ is nonzero at $\left(y_{i}^{*}, y_{j}^{*}\right)$, and the derivatives in (43) and (44) are well defined.

Suppose now that $\frac{\partial^{2} \hat{g}_{i}}{\partial x_{i}^{2}}$ is nonzero for $i=1,2$ (the case $\frac{\partial^{2} \hat{g}_{i}}{\partial x_{i}^{2}}=0$ is considered below in the proof of Proposition 1). Then, a careful reorganization of terms in the expression of $\frac{d y_{j}^{*}}{d x_{j}}$ in (43) leads to

$$
\frac{d y_{j}^{*}}{d x_{j}}=\frac{-\frac{\partial^{2} \hat{g}_{j}}{\partial x_{j}^{2}}}{\frac{\partial^{2} \hat{g}_{j}}{\partial x_{j}^{2}}-\frac{\partial^{2} \hat{f}_{j}}{\partial y_{j}^{2}}}\left[1-\frac{\frac{\partial^{2} \hat{g}_{j}}{\partial x_{j} \partial y_{i}}}{-\frac{\partial^{2} \hat{g}_{j}}{\partial x_{j}^{2}}} \frac{N_{j j}}{\Delta}\right],
$$

where $N_{j j}=\frac{\partial^{2} \hat{g}_{i}}{\partial x_{i} \partial y_{j}} \frac{\partial^{2} \hat{g}_{j}}{\partial x_{j}^{2}}-\left(\frac{\partial^{2} \hat{g}_{j}}{\partial x_{j}^{2}}-\frac{\partial^{2} \hat{f}_{j}}{\partial y_{j}^{2}}\right) \frac{\partial^{2} \hat{g}_{i}}{\partial x_{i} \partial x_{j}}$

\footnotetext{
${ }^{51}$ From the expression in (2), the argument $s_{i}=x_{i}+y_{i}$ of the gross profit function $g_{i}$, hence also of the reduced-form $\hat{g}_{i}$, implies that inequalities $(6-7)$ can be rewritten by substituting the derivatives with respect to $x_{i}$ for the ones with respect to $y_{i}$. Thus $\partial^{2} \hat{g}_{i} / \partial y_{i} \partial x_{i}=\partial^{2} \hat{g}_{i} / \partial x_{i}^{2}, \partial^{2} \hat{g}_{i} / \partial y_{i} \partial x_{j}=\partial^{2} \hat{g}_{i} / \partial x_{i} \partial x_{j}$, and $\partial^{2} \hat{g}_{i} / \partial y_{i} \partial y_{j}=\partial^{2} \hat{g}_{i} / \partial x_{i} \partial y_{j}$, for $i, j=1,2, j \neq i$. We make use of these substitutions throughout the appendix.
} 
Similarly, a reorganization of terms in the expression of $\frac{d y_{i}^{*}}{d x_{j}}$ in (44) leads to

$$
\frac{d y_{i}^{*}}{d x_{j}}=\frac{-\frac{\partial^{2} \hat{g}_{i}}{\partial x_{i} \partial x_{j}}}{\frac{\partial^{2} \hat{g}_{i}}{\partial x_{i}^{2}}-\frac{\partial^{2} \hat{f}_{i}}{\partial y_{i}^{2}}}\left[1-\frac{\frac{\partial^{2} \hat{g}_{i}}{\partial x_{i} \partial y_{j}}}{-\frac{\partial^{2} \hat{g}_{i}}{\partial x_{i} \partial x_{j}}} \frac{N_{i j}}{\Delta}\right]
$$

where $N_{i j}=\frac{\partial^{2} \hat{g}_{j}}{\partial x_{j} \partial y_{i}} \frac{\partial^{2} \hat{g}_{i}}{\partial x_{i} \partial x_{j}}-\left(\frac{\partial^{2} \hat{g}_{i}}{\partial x_{i}^{2}}-\frac{\partial^{2} \hat{f}_{i}}{\partial y_{i}^{2}}\right) \frac{\partial^{2} \hat{g}_{j}}{\partial x_{j}^{2}}$.

Both expressions in (46) and (47) are well defined because the denominators of their respective first terms are nonzero by assumption (second-order condition for a unique $\mathbf{y}^{*}(\mathbf{x})$ ).

\section{A.2 Proof of Proposition 1.}

We want to establish that $\frac{d y_{i}^{*}}{d x_{i}}<0 \Leftrightarrow \frac{\partial^{2} \hat{g}_{i}}{\partial s_{i}^{2}}<0$, where $s_{i}=x_{i}+y_{i}$, and with $\hat{g}_{i}=\hat{g}_{i}\left(x_{i}+y_{i}^{*}, x_{j}, y_{j}^{*}\right)$, $\hat{g}_{j}=\hat{g}_{j}\left(x_{j}+y_{j}^{*}, x_{i}, y_{i}^{*}\right), \hat{f}_{i}=\hat{f}_{i}\left(y_{i}^{*}\right)$, and $\hat{f}_{j}=\hat{f}_{j}\left(y_{j}^{*}\right), i, j=1,2, j \neq i$, throughout this section for clarity. There are three cases:

Case 1: $\frac{\partial^{2} \hat{g}_{i}}{\partial s_{i}^{2}}=0$. Recalling from $s_{i}=x_{i}+y_{i}$ in $\hat{g}_{i}$ that $\frac{\partial^{2} \hat{g}_{i}}{\partial y_{i} \partial x_{i}}=\frac{\partial^{2} \hat{g}_{i}}{\partial x_{i}^{2}}$ and $\frac{\partial^{2} \hat{g}_{i}}{\partial y_{i} \partial x_{j}}=\frac{\partial^{2} \hat{g}_{i}}{\partial x_{i} \partial x_{j}}$, from (6) we have $\left\|\frac{\partial^{2} \hat{g}_{i}}{\partial y_{i} \partial x_{i}}\right\|=\left\|\frac{\partial^{2} \hat{g}_{i}}{\partial x_{i}^{2}}\right\|=0 \geq\left\|\frac{\partial^{2} \hat{g}_{i}}{\partial y_{i} \partial x_{j}}\right\|=\left\|\frac{\partial^{2} \hat{g}_{i}}{\partial x_{i} \partial x_{j}}\right\|$, implying that $\frac{\partial^{2} \hat{g}_{i}}{\partial x_{i} \partial x_{j}}=0$. Then, by inserting $\frac{\partial^{2} \hat{g}_{j}}{\partial x_{j}^{2}}=0$ and $\frac{\partial^{2} \hat{g}_{i}}{\partial x_{i} \partial x_{j}}=0$ into (43) and (44) we find

$$
\frac{d y_{j}^{*}}{d x_{j}}=\frac{d y_{i}^{*}}{d x_{j}}=0
$$

Case 2: $\frac{\partial^{2} \hat{g}_{i}}{\partial s_{i}^{2}}<0$. (i) Here $\frac{\partial^{2} \hat{f}_{i}\left(y_{i}^{*}\right)}{\partial y_{i}^{2}} \geq 0$ implies that $\frac{\partial^{2} \hat{g}_{i}}{\partial x_{i}^{2}}-\frac{\partial^{2} \hat{f}_{i}}{\partial y_{i}^{2}} \leq \frac{\partial^{2} \hat{g}_{i}}{\partial x_{i}^{2}}<0$, while $\left\|\frac{\partial^{2} \hat{g}_{i}}{\partial x_{i}^{2}}\right\| \geq\left\|\frac{\partial^{2} \hat{g}_{i}}{\partial x_{i} \partial x_{j}}\right\|$ by assumption from (7) implies that $\frac{\partial^{2} \hat{g}_{i}}{\partial x_{i}^{2}} \leq \frac{\partial^{2} \hat{g}_{i}}{\partial x_{i} \partial x_{j}} \leq 0$, and by transitivity $\frac{\partial^{2} \hat{g}_{i}}{\partial x_{i}^{2}}-\frac{\partial^{2} \hat{f}_{i}}{\partial y_{i}^{2}} \leq \frac{\partial^{2} \hat{g}_{i}}{\partial x_{i} \partial x_{j}} \leq 0$. (ii) As $\frac{\partial^{2} \hat{\mathcal{g}}_{j}}{\partial x_{j}^{2}}<0$, here $\left\|\frac{\partial^{2} \hat{g}_{j}}{\partial x_{j}^{2}}\right\| \geq\left\|\frac{\partial^{2} \hat{g}_{j}}{\partial x_{j} \partial y_{i}}\right\|$ by assumption from (6) leads to $\frac{\partial^{2} \hat{\mathscr{g}}_{j}}{\partial x_{j}^{2}} \leq \frac{\partial^{2} \hat{\mathcal{g}}_{j}}{\partial x_{j} \partial y_{i}} \leq 0$. From (i) and (ii) we obtain that

$$
-\left(\frac{\partial^{2} \hat{g}_{i}}{\partial x_{i}^{2}}-\frac{\partial^{2} \hat{f}_{i}}{\partial y_{i}^{2}}\right) \frac{\partial^{2} \hat{g}_{j}}{\partial x_{j}^{2}} \leq-\frac{\partial^{2} \hat{g}_{i}}{\partial x_{i} \partial x_{j}} \frac{\partial^{2} \hat{g}_{j}}{\partial x_{j} \partial y_{i}} \leq 0,
$$

with an equality sign (the 1st one) if and only if $\frac{\partial^{2} \hat{g}_{j}}{\partial x_{j}^{2}}=\frac{\partial^{2} \hat{g}_{j}}{\partial x_{j} \partial y_{i}}<0$ and $\frac{\partial^{2} \hat{g}_{i}}{\partial x_{i}^{2}}-\frac{\partial^{2} \hat{f}_{i}}{\partial y_{i}^{2}}=\frac{\partial^{2} \hat{g}_{i}}{\partial x_{i} \partial x_{j}}<0$. Multiplying through by $\frac{\partial^{2} \hat{\mathcal{g}}_{i}}{\partial x_{j}^{2}}-\frac{\partial^{2} \hat{f}_{i}}{\partial y_{j}^{2}}<0$, adding $\frac{\partial^{2} \hat{\mathcal{g}}_{j}}{\partial x_{j}^{2}} \frac{\partial^{2} \hat{\mathcal{g}}_{j}}{\partial x_{j} \partial y_{i}} \frac{\partial^{2} \hat{g}_{i}}{\partial x_{i} \partial y_{j}}$ on both sides, and reorganizing terms, leads to

$$
1 \geq \frac{\frac{\partial^{2} \hat{g}_{j}}{\partial x_{j} \partial y_{i}}}{-\frac{\partial^{2} \hat{g}_{j}}{\partial x_{j}^{2}}} \frac{N_{j j}}{\Delta}
$$


so that the expression between brackets is non-negative (46) is positive, and finally we have

$$
\frac{d y_{j}^{*}}{d x_{j}}=-\underbrace{\frac{-\frac{\partial^{2} \hat{g}_{j}}{\partial x_{j}^{2}}}{\frac{\partial^{2} \hat{g}_{j}}{\partial x_{j}^{2}}-\frac{\partial^{2} \hat{f}_{j}}{\partial y_{j}^{2}}}}_{<0} \underbrace{\left[1-\frac{\frac{\partial^{2} \hat{g}_{j}}{\partial x_{j} \partial y_{i}}}{-\frac{\partial^{2} \hat{g}_{j}}{\partial x_{j}^{2}}} \frac{N_{j j}}{\Delta}\right]}_{\geq 0} \leq 0,
$$

again with an equality sign if and only if $\frac{\partial^{2} \hat{g}_{j}}{\partial x_{j}^{2}}=\frac{\partial^{2} \hat{g}_{j}}{\partial x_{j} \partial y_{i}}<0$ and $\frac{\partial^{2} \hat{g}_{i}}{\partial x_{i}^{2}}-\frac{\partial^{2} \hat{f}_{i}}{\partial y_{i}^{2}}=\frac{\partial^{2} \hat{g}_{i}}{\partial x_{i} \partial x_{j}}<0$, where the latter equality implies that $\frac{\partial^{2} \hat{f}_{i}}{\partial y_{i}^{2}}=0$.

Case 3: $\frac{\partial^{2} \hat{g}_{i}}{\partial s_{i}^{2}}>0(i=1,2)$. Here we have $\left(\frac{\partial^{2} \hat{g}_{i}}{\partial x_{i}^{2}}-\frac{\partial^{2} \hat{f}_{i}}{\partial y_{i}^{2}}\right) \frac{\partial^{2} \hat{g}_{j}}{\partial x_{j}^{2}}<0$, which together with $\frac{\partial^{2} \hat{g}_{j}}{\partial x_{j} \partial y_{i}} \frac{\partial^{2} \hat{g}_{i}}{\partial x_{i} \partial x_{j}} \geq 0$ (partial cross-derivatives have the same sign by assumption) implies that $\left(\frac{\partial^{2} \hat{g}_{i}}{\partial x_{i}^{2}}-\frac{\partial^{2} \hat{f}_{i}}{\partial y_{i}^{2}}\right) \frac{\partial^{2} \hat{g}_{j}}{\partial x_{j}^{2}}<\frac{\partial^{2} \hat{g}_{j}}{\partial x_{j} \partial y_{i}} \frac{\partial^{2} \hat{g}_{i}}{\partial x_{i} \partial x_{j}}$. Multiplying through by $\frac{\partial^{2} \hat{g}_{j}}{\partial x_{j}^{2}}-\frac{\partial^{2} \hat{f}_{j}}{\partial y_{j}^{2}}<0$, adding $-\frac{\partial^{2} \hat{g}_{j}}{\partial x_{j}^{2}} \frac{\partial^{2} \hat{g}_{j}}{\partial x_{j} \partial y_{i}} \frac{\partial^{2} \hat{g}_{i}}{\partial x_{i} \partial y_{j}}$ on both sides of the inequality sign, and reorganizing terms, leads to

$$
1>\frac{\frac{\partial^{2} \hat{g}_{j}}{\partial x_{j} \partial y_{i}}}{-\frac{\partial^{2} \hat{g}_{j}}{\partial x_{j}^{2}}} \frac{N_{j j}}{\Delta},
$$

so that the expression between brackets in (46) is positive. Then $-\frac{\partial^{2} \hat{g}_{j}}{\partial x_{j}^{2}}\left(\frac{\partial^{2} \hat{g}_{j}}{\partial x_{j}^{2}}-\frac{\partial^{2} \hat{f}_{j}}{\partial y_{j}^{2}}\right)^{-1}>0$ implies finally that

$$
\frac{d y_{j}^{*}}{d x_{j}}=\underbrace{\frac{-\frac{\partial^{2} \hat{g}_{j}}{\partial x_{j}^{2}}}{\frac{\partial^{2} \hat{g}_{j}}{\partial x_{j}^{2}}-\frac{\partial^{2} \hat{f}_{j}}{\partial y_{j}^{2}}}}_{>0} \underbrace{\left[1-\frac{\frac{\partial^{2} \hat{g}_{j}}{\partial x_{j} \partial y_{i}}}{-\frac{\partial^{2} \hat{g}_{j}}{\partial x_{j}^{2}}} \frac{N_{j j}}{\Delta}\right]}_{>0}>0 .
$$

The sign of $\frac{d y_{j}^{*}}{d x_{j}}$ in (48), (49) and (51), establishes Proposition 1.

\section{A.3 Lemmas}

The technical results introduced in this section are needed to prove Propositions 2, 3, and 5. The first two lemmas establish a connection between properties of $\hat{g}_{i}(\mathbf{x}, \mathbf{y})$ and $\hat{g}_{i}\left(\mathbf{x}, \mathbf{y}^{*}(\mathbf{x})\right)-\hat{f}_{i}\left(y_{i}^{*}(\mathbf{x})\right)$.

Lemma A.1 Suppose that $\frac{\partial^{2} \hat{g}_{i}}{\partial x_{i}^{2}} \leq 0, i=1,2$. Then $\frac{d\left[\hat{g}_{i}\left(x_{i}+y_{i}^{*}, x_{j}, y_{j}^{*}\right)-\hat{f}_{i}\left(y_{i}^{*}\right)\right]}{d x_{j}}, i, j=1,2, j \neq i$, has the same sign as $\frac{\partial \hat{g}_{i}\left(x_{i}+y_{i}, x_{j}, y_{j}\right)}{\partial x_{j}}$ and $\frac{\partial \hat{g}_{i}\left(x_{i}+y_{i}, x_{j}, y_{j}\right)}{\partial y_{j}}$. 
Proof. By the envelope theorem, as $y_{i}^{*}=y_{i}^{*}\left(x_{i}, x_{j}\right)$ maximizes $\hat{g}_{i}\left(x_{i}+y_{i}, x_{j}, y_{j}\right)-\hat{f}_{i}\left(y_{i}\right)$, we have

$$
\frac{d\left[\hat{g}_{i}\left(x_{i}+y_{i}^{*}, x_{j}, y_{j}^{*}\right)-\hat{f}_{i}\left(y_{i}^{*}\right)\right]}{d x_{j}}=\frac{\partial \hat{g}_{i}\left(x_{i}+y_{i}^{*}, x_{j}, y_{j}^{*}\right)}{\partial x_{j}}+\frac{\partial \hat{g}_{i}\left(x_{i}+y_{i}^{*}, x_{j}, y_{j}^{*}\right)}{\partial y_{j}} \frac{d y_{j}^{*}}{d x_{j}}
$$

Our objective is to determine the sign of the RHS expression in (52). Given the (same) sign of $\frac{\partial \hat{g}_{i}\left(x_{i}+y_{i}^{*}, x_{j}, y_{j}^{*}\right)}{\partial x_{j}}$ and $\frac{\partial \hat{g}_{i}\left(x_{i}+y_{i}^{*}, x_{j}, y_{j}^{*}\right)}{\partial y_{j}}$, we need only characterizing $\frac{d y_{j}^{*}}{d x_{j}}$.

First, if $\frac{\partial^{2} \hat{g}_{i}}{\partial x_{i}^{2}}=0(i=1,2)$, we know from $(48)$ that $\frac{d y_{j}^{*}}{d x_{j}}=0$, which is sufficient to conclude.

Next, if $\frac{\partial^{2} \hat{g}_{i}}{\partial x_{i}^{2}}<0(i=1,2)$, we know from (49) that $\frac{d y_{j}^{*}}{d x_{j}} \leq 0$. Then toward a contradiction we suppose that $\left\|\frac{d y_{j}^{*}}{d x_{j}}\right\|>1$, or equivalently here $\frac{d y_{j}^{*}}{d x_{j}}<-1$. Developing the expression in (46) then leads to

$$
\frac{\partial^{2} \hat{g}_{j}}{\partial x_{j} \partial y_{i}}\left(\frac{\partial^{2} \hat{g}_{i}}{\partial x_{i} \partial y_{j}}-\frac{\partial^{2} \hat{g}_{i}}{\partial x_{i} \partial x_{j}}\right)>-\underbrace{\frac{\partial^{2} \hat{f}_{j}}{\partial y_{j}^{2}}}_{\geq 0} \underbrace{\left(\frac{\partial^{2} \hat{g}_{i}}{\partial x_{i}^{2}}-\frac{\partial^{2} \hat{f}_{i}\left(y_{i}^{*}\right)}{\partial y_{i}^{2}}\right)}_{<0} .
$$

As $\frac{\partial^{2} \hat{g}_{i}}{\partial x_{i} \partial y_{j}}$ and $\frac{\partial^{2} \hat{g}_{i}}{\partial x_{i} \partial x_{j}}$ have the same sign, and $\left\|\frac{\partial^{2} \hat{g}_{i}}{\partial x_{i} \partial x_{j}}\right\| \geq\left\|\frac{\partial^{2} \hat{g}_{i}}{\partial x_{i} \partial y_{j}}\right\|$ (model specifications in (6-7)), we know that the expression on the LHS of the strict inequality sign in (A.9) is non-positive. However, $\frac{\partial^{2} \hat{f}_{j}}{\partial y_{j}^{2}} \geq 0$ (by assumption) and $\frac{\partial^{2} \hat{g}_{i}}{\partial x_{i}^{2}}-\frac{\partial^{2} \hat{f}_{i}\left(y_{i}^{*}\right)}{\partial y_{i}^{2}}<0$ (second-order condition) imply that the product on the RHS is always non-negative, a contradiction. Hence $\left\|\frac{d y_{j}^{*}}{d x_{j}}\right\| \leq 1$. This, together with $\left\|\frac{\partial \hat{g}_{i}\left(x_{i}+y_{i}^{*}, x_{j}, y_{j}^{*}\right)}{\partial x_{j}}\right\| \geq\left\|\frac{\partial \hat{g}_{i}\left(x_{i}+y_{i}^{*}, x_{j}, y_{j}^{*}\right)}{\partial y_{j}}\right\|$ (model specifications), is sufficient to conclude that $\frac{d\left[\hat{g}_{i}\left(x_{i}+y_{i}^{*}, x_{j}, y_{j}^{*}\right)-\hat{f}_{i}\left(y_{i}^{*}\right)\right]}{d x_{j}}$ in (52) has the same sign as $\frac{\partial \hat{g}_{i}\left(x_{i}+y_{i}, x_{j}, y_{j}\right)}{\partial x_{j}}$ and $\frac{\partial \hat{g}_{i}\left(x_{i}+y_{i}, x_{j}, y_{j}\right)}{\partial y_{j}}$.

Lemma A.2 Suppose that $\frac{\partial^{2} \hat{g}_{i}}{\partial x_{i}^{2}} \leq 0, i=1$, 2. If $\frac{\partial \hat{g}_{i}\left(x_{i}+y_{i}, x_{j}, y_{j}\right)}{\partial x_{j}} \geq 0$ and $\frac{\partial \hat{g}_{i}\left(x_{i}+y_{i}, x_{j}, y_{j}\right)}{\partial y_{j}} \geq 0, i, j=1,2, j \neq i$, then $\frac{d\left[\hat{g}_{i}\left(x_{i}+y_{i}^{*}, x_{j}, y_{j}^{*}\right)-\hat{f}_{i}\left(y_{i}^{*}\right)\right]}{d x_{i}} \geq 0$ also.

Proof. By the envelope theorem, as $y_{i}^{*}=y_{i}^{*}\left(x_{i}, x_{j}\right)$ maximizes $\hat{g}_{i}\left(x_{i}+y_{i}, x_{j}, y_{j}\right)-\hat{f}_{i}\left(y_{i}\right)$, we have

$$
\frac{d\left[\hat{g}_{i}\left(x_{i}+y_{i}^{*}, x_{j}, y_{j}^{*}\right)-\hat{f}_{i}\left(y_{i}^{*}\right)\right]}{d x_{i}}=\frac{\partial \hat{g}_{i}\left(x_{i}+y_{i}^{*}, x_{j}, y_{j}^{*}\right)}{\partial x_{i}}+\frac{\partial \hat{g}_{i}\left(x_{i}+y_{i}^{*}, x_{j}, y_{j}^{*}\right)}{\partial y_{j}} \frac{d y_{j}^{*}}{d x_{i}}
$$

where $\frac{\partial \hat{g}_{i}\left(x_{i}+y_{i}^{*}, x_{j}, y_{j}^{*}\right)}{\partial x_{i}} \geq 0$ as a model specification, and $\frac{\partial \hat{g}_{i}\left(x_{i}+y_{i}^{*}, x_{j}, y_{j}^{*}\right)}{\partial y_{j}} \geq 0$ as an assumption of the present lemma. In order to determine the sign of the RHS expression in (54), we thus need only characterizing $\frac{d y_{j}^{*}}{d x_{i}}$.

First, if $\frac{\partial^{2} \hat{g}_{i}}{\partial x_{i}^{2}}=0(i=1,2)$, we know from (48) that $\frac{d y_{j}^{*}}{d x_{i}}=0$, which is sufficient to conclude. 
Next, if $\frac{\partial^{2} \hat{g}_{i}}{\partial x_{i}^{2}}<0(i=1,2)$, recall from (47) in Section A.1 that

$$
\frac{d y_{j}^{*}}{d x_{i}}=\frac{-\frac{\partial^{2} \hat{g}_{j}}{\partial x_{j} \partial x_{i}}}{\frac{\partial^{2} \hat{g}_{j}}{\partial x_{j}^{2}}-\frac{\partial^{2} \hat{f}_{j}}{\partial y_{j}^{2}}}\left[1-\frac{\frac{\partial^{2} \hat{g}_{j}}{\partial x_{j} \partial y_{i}}}{-\frac{\partial^{2} \hat{g}_{j}}{\partial x_{j} \partial x_{i}}} \frac{N_{j i}}{\Delta}\right]
$$

where $N_{j i}=\frac{\partial^{2} \hat{g}_{i}}{\partial x_{i} \partial y_{j}} \frac{\partial^{2} \hat{g}_{j}}{\partial x_{j} \partial x_{i}}-\left(\frac{\partial^{2} \hat{g}_{j}}{\partial x_{j}^{2}}-\frac{\partial^{2} \hat{f}_{j}}{\partial y_{j}^{2}}\right) \frac{\partial^{2} \hat{g}_{i}}{\partial x_{i}^{2}}$. There are two possible cases that depend on the sign of $\frac{\partial^{2} \hat{g}_{j}}{\partial x_{j} \partial x_{i}}$.

(i) If $\frac{\partial^{2} \hat{g}_{j}}{\partial x_{j} \partial x_{i}} \geq 0(i, j=1,2, j \neq i)$, as $\frac{\partial^{2} \hat{g}_{j}}{\partial x_{j}^{2}}-\frac{\partial^{2} \hat{f}_{j}}{\partial y_{j}^{2}}<0$ we have $-\frac{\partial^{2} \hat{g}_{j}}{\partial x_{j} \partial x_{i}} /\left(\frac{\partial^{2} \hat{g}_{j}}{\partial x_{j}^{2}}-\frac{\partial^{2} \hat{f}_{j}}{\partial y_{j}^{2}}\right) \geq 0$. Then, toward a contradiction, suppose that the expression between brackets in (55) is negative. This, together with $\left\|\frac{\partial^{2} \hat{g}_{j}}{\partial x_{j} \partial y_{i}} / \frac{\partial^{2} \hat{g}_{j}}{\partial x_{j} \partial x_{i}}\right\| \leq 1$ (model specifications in (6-7)), implies that $\frac{N_{j i}}{\Delta}<-1$, which can be rewritten as

$$
\frac{\partial^{2} \hat{g}_{i}}{\partial x_{i} \partial y_{j}}\left(\frac{\partial^{2} \hat{g}_{j}}{\partial x_{j} \partial x_{i}}-\frac{\partial^{2} \hat{g}_{j}}{\partial x_{j} \partial y_{i}}\right)<\frac{\partial^{2} \hat{f}_{i}}{\partial y_{i}^{2}}\left(\frac{\partial^{2} \hat{g}_{j}}{\partial x_{j}^{2}}-\frac{\partial^{2} \hat{f}_{j}}{\partial y_{j}^{2}}\right)
$$

where the expression on the LHS of the inequality sign is non-negative, whereas the expression on the RHS is non-positive, a contradiction. It follows that $\frac{d y_{j}^{*}}{d x_{i}} \geq 0$, which is sufficient to conclude directly that $\frac{d\left[\hat{g}_{i}\left(x_{i}+y_{i}^{*}, x_{j}, y_{j}^{*}\right)-\hat{f}_{i}\left(y_{i}^{*}\right)\right]}{d x_{i}}$ in (54) is non-negative also.

(ii) If $\frac{\partial^{2} \hat{g}_{j}}{\partial x_{j} \partial x_{i}}<0(i, j=1,2, j \neq i)$, unlike in the previous case the model specifications do not imply that $\frac{d y_{j}^{*}}{d x_{i}} \geq 0$. Then, toward a contradiction, whenever $\frac{d y_{j}^{*}}{d x_{i}}<0$ suppose that $\left\|\frac{d y_{j}^{*}}{d x_{i}}\right\|>1$, or equivalently here $-\frac{d y_{j}^{*}}{d x_{i}}>1$. From (55), by using $\Delta>0$ (see (42) and related subsequent comments in Section A.1) we have

$$
\frac{-\frac{\partial^{2} \hat{g}_{j}}{\partial x_{j} \partial y_{i}} \frac{\partial^{2} \hat{g}_{i}}{\partial x_{i}^{2}}+\frac{\partial^{2} \hat{g}_{j}}{\partial x_{j} \partial x_{i}}\left(\frac{\partial^{2} \hat{g}_{i}}{\partial x_{i}^{2}}-\frac{\partial^{2} \hat{f}_{i}}{\partial y_{i}^{2}}\right)}{\left(\frac{\partial^{2} \hat{g}_{i}}{\partial x_{i}^{2}}-\frac{\partial^{2} \hat{f}_{i}}{\partial y_{i}^{2}}\right)\left(\frac{\partial^{2} \hat{g}_{j}}{\partial x_{j}^{2}}-\frac{\partial^{2} \hat{f}_{j}}{\partial y_{j}^{2}}\right)-\frac{\partial^{2} \hat{g}_{i}}{\partial x_{i} \partial y_{j}} \frac{\partial^{2} \hat{g}_{j}}{\partial x_{j} \partial y_{i}}}>1,
$$

which can be rewritten as

$$
-\frac{\partial^{2} \hat{g}_{j}}{\partial x_{j} \partial y_{i}} \frac{\partial^{2} \hat{g}_{i}}{\partial x_{i}^{2}}+\frac{\partial^{2} \hat{g}_{j}}{\partial x_{j} \partial x_{i}}\left(\frac{\partial^{2} \hat{g}_{i}}{\partial x_{i}^{2}}-\frac{\partial^{2} \hat{f}_{i}}{\partial y_{i}^{2}}\right)>\left(\frac{\partial^{2} \hat{g}_{i}}{\partial x_{i}^{2}}-\frac{\partial^{2} \hat{f}_{i}}{\partial y_{i}^{2}}\right)\left(\frac{\partial^{2} \hat{g}_{j}}{\partial x_{j}^{2}}-\frac{\partial^{2} \hat{f}_{j}}{\partial y_{j}^{2}}\right)-\frac{\partial^{2} \hat{g}_{i}}{\partial x_{i} \partial y_{j}} \frac{\partial^{2} \hat{g}_{j}}{\partial x_{j} \partial y_{i}}
$$

with $\frac{\partial^{2} \hat{g}_{i}}{\partial x_{i}^{2}}$ and $\frac{\partial^{2} \hat{g}_{i}}{\partial x_{i} \partial y_{j}}$ both negative here. Moreover we know by assumption from (7) that $\left\|\frac{\partial^{2} \hat{g}_{i}}{\partial x_{i}^{2}}\right\| \geq$ $\left\|\frac{\partial^{2} \hat{g}_{i}}{\partial x_{i} \partial y_{j}}\right\|^{i}$. Therefore, substituting $\frac{\partial^{2} \hat{g}_{i}}{\partial x_{i}^{2}}$ for $\frac{\partial^{2} \hat{g}_{i}}{\partial x_{i} \partial y_{j}}$ in the last term above, by transitivity we obtain

$$
-\frac{\partial^{2} \hat{g}_{j}}{\partial x_{j} \partial y_{i}} \frac{\partial^{2} \hat{g}_{i}}{\partial x_{i}^{2}}+\frac{\partial^{2} \hat{g}_{j}}{\partial x_{j} \partial x_{i}}\left(\frac{\partial^{2} \hat{g}_{i}}{\partial x_{i}^{2}}-\frac{\partial^{2} \hat{f}_{i}}{\partial y_{i}^{2}}\right)>\left(\frac{\partial^{2} \hat{g}_{i}}{\partial x_{i}^{2}}-\frac{\partial^{2} \hat{f}_{i}}{\partial y_{i}^{2}}\right)\left(\frac{\partial^{2} \hat{g}_{j}}{\partial x_{j}^{2}}-\frac{\partial^{2} \hat{f}_{j}}{\partial y_{j}^{2}}\right)-\frac{\partial^{2} \hat{g}_{j}}{\partial x_{j} \partial y_{i}} \frac{\partial^{2} \hat{g}_{i}}{\partial x_{i}^{2}}
$$


which simplifies to

$$
\frac{\partial^{2} \hat{g}_{j}}{\partial x_{j} \partial x_{i}}<\frac{\partial^{2} \hat{g}_{j}}{\partial x_{j}^{2}}-\frac{\partial^{2} \hat{f}_{j}}{\partial y_{j}^{2}} \leq \frac{\partial^{2} \hat{g}_{j}}{\partial x_{j}^{2}} \leq 0 .
$$

As the latter inequalities contradict the initial assumption in (6) that $\left\|\frac{\partial^{2} \hat{g}_{j}}{\partial x_{j} \partial x_{i}}\right\| \leq\left\|\frac{\partial^{2} \hat{g}_{j}}{\partial x_{j}^{2}}\right\|$, it must be the case that $\left\|\frac{d y_{j}^{*}}{d x_{i}}\right\| \leq 1$. This, together with the model specification in (4-5) that $\left\|\frac{\partial \hat{g}_{i}\left(x_{i}+y_{i}^{*}, x_{j}, y_{j}^{*}\right)}{\partial x_{i}}\right\| \geq$ $\left\|\frac{\partial \hat{g}_{i}\left(x_{i}+y_{i}^{*}, x_{j}, y_{j}^{*}\right)}{\partial y_{j}}\right\|$, is sufficient to conclude that $\frac{d\left[\hat{g}_{i}\left(x_{i}+y_{i}^{*}, x_{j}, y_{j}^{*}\right)-\hat{f}_{i}\left(y_{i}^{*}\right)\right]}{d x_{i}}$ in (54) is non-negative.

The next two lemmas were established in Laussel and Le Breton (2001). We restate them in the notation of this paper for a self-contained appendix: ${ }^{52}$

Lemma A.3 If $v$ is superadditive, that is $\Lambda \geq v(\{1\})+v(\{2\})$, then in all TSPNE $v_{0}^{*}=0$, and all vectors of equilibrium profits $\left(v_{1}^{*}, v_{2}^{*}\right)$ are such that $v_{1}^{*}+v_{2}^{*}=\Lambda$.

Lemma A.4 If $v$ is strictly subadditive, that is $\Lambda<v(\{1\})+v(\{2\})$, then in all TSPNE $v_{0}^{*}>0$, and there exists a unique vector of equilibrium profits $\left(v_{1}^{*}, v_{2}^{*}\right)$, where $v_{i}^{*}=\Lambda-v(\{j\}), i, j=1,2, j \neq i$.

\section{A.4 Proof of Propositions 2 and 3.}

Proof of Proposition 2. We first use Lemmas A.1 and A.2, and finally Lemma A.3, in order to extend a proof by Billette de Villemeur and Versaevel (2003, Proposition 1) to establish the (weak) superadditivity of $v($.$) . Then we show that the equilibrium payoffs \left(v_{1}^{*}, v_{2}^{*}\right)$ exist that are (weakly) higher than the respective standalone values $\left(\underline{v}_{1}, \underline{v}_{2}\right)$.

1) Denote by $X_{\{i\}}^{*}$ the set of the lab's actions that maximize its expected joint profit with firm $i$, that is

$$
X_{\{i\}}^{*}=\arg \max _{\mathbf{x}}\left(\max _{y_{i}}\left[\hat{g}_{i}\left(x_{i}+y_{i}, x_{j}, y_{j}\right)-\hat{f}_{i}\left(y_{i}\right)\right]-\hat{f}_{0}(\mathbf{x})\right)
$$

Define $\mathbf{a}=\left(a_{1}, a_{2}\right) \in X_{\{1\}}^{*}$ and $\mathbf{b}=\left(b_{1}, b_{2}\right) \in X_{\{2\}}^{*}$. We know from the initial model specifications that $\frac{\partial \hat{g}_{i}\left(x_{i}+y_{i}, x_{j}, y_{j}\right)}{\partial s_{i}} \geq 0$, where $s_{i}=x_{i}+y_{i}$. Moreover, it is assumed in this proposition that $\frac{\partial^{2} \hat{g}_{i}}{\partial x_{i}^{2}} \leq 0$ (non-increasing returns to R\&D), and from (13) that $\frac{\partial \hat{g}_{i}\left(x_{i}+y_{i}, x_{j}, y_{j}\right)}{\partial x_{j}} \geq 0$ and $\frac{\partial \hat{g}_{i}\left(x_{i}+y_{i}, x_{j}, y_{j}\right)}{\partial y_{j}} \geq 0$ (nonnegative R\&D externalities). It follows that $\hat{g}_{i}\left(x_{i}+y_{i}^{*}(\mathbf{x}), x_{j}, y_{j}^{*}(\mathbf{x})\right)-\hat{f}_{i}\left(y_{i}^{*}(\mathbf{x})\right)$ is non-decreasing in $x_{j}$ from Lemma A.1, and also in $x_{i}$ from Lemma A.2. Therefore, for $\mathbf{u}=\left(u_{1}, u_{2}\right)$, with $u_{1}=a_{1} \vee b_{1}$

\footnotetext{
${ }^{52}$ With two principals, the convexity condition introduced in Laussel and Le Breton (2001, Proposition 3.2, p. 103) coincides with the superadditivity of $v$ in our model, and the strong subadditivity property (Proposition 3.3, p. 104) coincides here with strict subadditivity.
} 
and $u_{2}=a_{2} \vee b_{2}$, we have

$$
\begin{gathered}
\hat{g}_{1}\left(a_{1}+y_{1}^{*}(\mathbf{a}), a_{2}, y_{2}^{*}(\mathbf{a})\right)-\hat{f}_{1}\left(y_{1}^{*}(\mathbf{a})\right) \leq \hat{g}_{1}\left(u_{1}+y_{1}^{*}(\mathbf{u}), u_{2}, y_{2}^{*}(\mathbf{u})\right)-\hat{f}_{1}\left(y_{1}^{*}(\mathbf{u})\right), \\
\hat{g}_{2}\left(b_{2}+y_{2}^{*}(\mathbf{b}), b_{1}, y_{1}^{*}(\mathbf{b})\right)-\hat{f}_{2}\left(y_{2}^{*}(\mathbf{b})\right) \leq \hat{g}_{2}\left(u_{2}+y_{2}^{*}(\mathbf{u}), u_{1}, y_{1}^{*}(\mathbf{u})\right)-\hat{f}_{2}\left(y_{2}^{*}(\mathbf{u})\right) .
\end{gathered}
$$

The non-negative cross-derivative in (12) implies the weak submodularity of $\hat{f}_{0}$ (Topkis, 1995). This property, with (56a) and (56b), together lead to

$$
\begin{aligned}
& \underbrace{\hat{g}_{1}\left(a_{1}+y_{1}^{*}(\mathbf{a}), a_{2}, y_{2}^{*}(\mathbf{a})\right)-\hat{f}_{1}\left(y_{1}^{*}(\mathbf{a})\right)-\hat{f}_{0}(\mathbf{a})}_{=v(\{1\})}+\underbrace{\hat{g}_{2}\left(b_{2}+y_{2}^{*}(\mathbf{b}), b_{1}, y_{1}^{*}(\mathbf{b})\right)-\hat{f}_{2}\left(y_{1}^{*}(\mathbf{b})\right)-\hat{f}_{0}(\mathbf{b})}_{=v(\{2\})} \\
\leq & \underbrace{\hat{g}_{1}\left(u_{1}+y_{1}^{*}(\mathbf{u}), u_{2}, y_{2}^{*}(\mathbf{u})\right)-\hat{f}_{1}\left(y_{1}^{*}(\mathbf{u})\right)+\hat{g}_{2}\left(u_{2}+y_{2}^{*}(\mathbf{u}), u_{1}, y_{1}^{*}(\mathbf{u})\right)-\hat{f}_{2}\left(y_{2}^{*}(\mathbf{u})\right)-\hat{f}_{0}(\mathbf{u})}_{\leq v(\{1,2\})}-\underbrace{\hat{f}_{0}(\mathbf{a} \wedge \mathbf{b})}_{\geq 0},
\end{aligned}
$$

which in turn establishes that $v(\{1\})+v(\{2\}) \leq v(\{1,2\})$. The conclusion that $v_{0}^{*}=0<v_{1}^{*}+v_{2}^{*}=$ $v(\{1,2\})$ follows directly from Lemma A.3.

2) To check that $v_{i}^{*} \geq \underline{v}_{i}$, recall that $\underline{v}_{i}=E_{\theta}\left[\tilde{g}_{j}\left(\mathbf{x}_{j}^{*}, \theta\right)\right]$, where $\mathbf{x}_{j}^{*} \in \arg \max _{\mathbf{x}} E_{\theta}\left[\tilde{g}_{j}(\mathbf{x}, \theta)-f_{0}(\mathbf{x}, \theta)\right]$ describes the lab's actions when firm $j$ has exclusive access to it (for $i, j=1,2, j \neq i$ ). Moreover, by assumption firm $i$ 's exclusive control of the lab dominates the standalone value, that is $v(\{i\}) \geq \underline{v}_{i}$. Then from the superadditivity of $v($.$) , together with v_{1}^{*}+v_{2}^{*}=v(\{1,2\})$ as established above, we have directly

$$
\underline{v}_{1}+\underline{v}_{2} \leq v(\{1\})+v(\{2\}) \leq v(\{1,2\})=v_{1}^{*}+v_{2}^{*},
$$

and the equilibrium set $\left\{\left(v_{1}^{*}, v_{2}^{*}\right) \mid v_{1}^{*}+v_{2}^{*}=\Lambda, v_{1}^{*} \geq \underline{v}_{1}, v_{2}^{*} \geq \underline{v}_{2}\right\}$ is nonempty.

Proof of Proposition 3. We first use Lemmas A.1 and A.4 to prove the strict subadditivity of $v($.$) , before establishing that the equilibrium payoffs \left(v_{1}^{*}, v_{2}^{*}\right)$ are (weakly) higher than the respective standalone values $\left(\underline{v}_{1}, \underline{v}_{2}\right)$.

1) Pick any $\mathbf{x}^{*}=\left(x_{1}^{*}, x_{2}^{*}\right)$ in $X_{\{1,2\}}^{*}$, the set of R\&D levels that maximize the expected industry profit.

It is assumed in this proposition that $\frac{\partial^{2} \hat{g}_{i}}{\partial x_{i}^{2}} \leq 0$ (non-increasing returns to R\&D), and from (17) that that $\frac{\partial \hat{g}_{i}\left(x_{i}+y_{i}, x_{j}, y_{j}\right)}{\partial x_{j}} \leq 0$ and $\frac{\partial \hat{g}_{i}\left(x_{i}+y_{i}, x_{j}, y_{j}\right)}{\partial y_{j}} \leq 0$ (weakly negative R\&D externalities). It follows from Lemma A. 1 that the net profit expression $\hat{g}_{i}\left(x_{i}+y_{i}^{*}(\mathbf{x}), x_{j}, y_{j}^{*}(\mathbf{x})\right)-\hat{f}_{i}\left(y_{i}^{*}(\mathbf{x})\right)$ is non-decreasing so that, for all $x_{1}^{*}, x_{2}^{*} \geq 0$,

$$
\begin{aligned}
& \hat{g}_{1}\left(x_{1}^{*}+y_{1}^{*}\left(\mathbf{x}^{*}\right), x_{2}^{*}, y_{2}^{*}\left(\mathbf{x}^{*}\right)\right)-\hat{f}_{1}\left(y_{1}^{*}\left(\mathbf{x}^{*}\right)\right) \leq \hat{g}_{1}\left(x_{1}^{*}+y_{1}^{*}\left(x_{1}^{*}, 0\right), 0, y_{2}^{*}\left(x_{1}^{*}, 0\right)\right)-\hat{f}_{1}\left(y_{1}^{*}\left(x_{1}^{*}, 0\right)\right), \\
& \hat{g}_{2}\left(x_{2}^{*}+y_{2}^{*}\left(\mathbf{x}^{*}\right), x_{1}^{*}, y_{1}^{*}\left(\mathbf{x}^{*}\right)\right)-\hat{f}_{2}\left(y_{2}^{*}\left(\mathbf{x}^{*}\right)\right) \leq \hat{g}_{2}\left(x_{2}^{*}+y_{2}^{*}\left(0, x_{2}^{*}\right), 0, y_{1}^{*}\left(0, x_{2}^{*}\right)\right)-\hat{f}_{2}\left(y_{2}^{*}\left(0, x_{2}^{*}\right)\right) .
\end{aligned}
$$

The negative cross-derivative in (16) implies the strict supermodularity of $\hat{f}_{0}$ (Topkis, 1995), with 
$\hat{f}_{0}(0,0)=0$. This property, together with (57a) and (57b), lead to

$$
\begin{gathered}
\underbrace{\hat{g}_{1}\left(x_{1}^{*}+y_{1}^{*}\left(\mathbf{x}^{*}\right), x_{2}^{*}, y_{2}^{*}\left(\mathbf{x}^{*}\right)\right)-\hat{f}_{1}\left(y_{1}^{*}\left(\mathbf{x}^{*}\right)\right)+\hat{g}_{2}\left(x_{2}^{*}+y_{2}^{*}\left(\mathbf{x}^{*}\right), x_{1}^{*}, y_{1}^{*}\left(\mathbf{x}^{*}\right)\right)-\hat{f}_{2}\left(y_{2}^{*}\left(\mathbf{x}^{*}\right)\right)-\hat{f}_{0}\left(x_{1}^{*}, x_{2}^{*}\right)}_{=v(\{1,2\})} \\
<\underbrace{\hat{g}_{1}\left(x_{1}^{*}+y_{1}^{*}\left(x_{1}^{*}, 0\right), 0, y_{2}^{*}\left(x_{1}^{*}, 0\right)\right)-\hat{f}_{1}\left(y_{1}^{*}\left(x_{1}^{*}, 0\right)\right)-\hat{f}_{0}\left(x_{1}^{*}, 0\right)}_{\leq v(\{1\})} \\
+\underbrace{\hat{g}_{2}\left(x_{2}^{*}+y_{2}^{*}\left(0, x_{2}^{*}\right), 0, y_{1}^{*}\left(0, x_{2}^{*}\right)\right)-\hat{f}_{2}\left(y_{2}^{*}\left(0, x_{2}^{*}\right)\right)-\hat{f}_{0}\left(0, x_{2}^{*}\right)}_{\leq v(\{2\})},
\end{gathered}
$$

which establishes that $v(\{1,2\})<v(\{1\})+v(\{2\})$. The conclusion that $v_{0}^{*}>0$ and $v_{i}^{*}=\Lambda-$ $v(\{j\}), i, j=1,2, j \neq i$, follows directly from Lemma A.4.

2) For any $\mathbf{x}_{1}^{*}$ and $\mathbf{x}_{2}^{*}$, by definition of $v($.$) we have$

$$
\begin{aligned}
& E_{\theta}\left[\tilde{g}_{1}\left(\mathbf{x}_{1}^{*}, \theta\right)+\tilde{g}_{2}\left(\mathbf{x}_{1}^{*}, \theta\right)-f_{0}\left(\mathbf{x}_{1}^{*}, \theta\right)\right] \leq v(\{1,2\}), \\
& E_{\theta}\left[\tilde{g}_{1}\left(\mathbf{x}_{2}^{*}, \theta\right)+\tilde{g}_{2}\left(\mathbf{x}_{2}^{*}, \theta\right)-f_{0}\left(\mathbf{x}_{2}^{*}, \theta\right)\right] \leq v(\{1,2\}) .
\end{aligned}
$$

Then by reorganizing terms, and recalling that $v(\{i\})=E_{\theta}\left[\tilde{g}_{i}\left(\mathbf{x}_{i}^{*}, \theta\right)-f_{0}\left(\mathbf{x}_{i}^{*}, \theta\right)\right]$ and $\underline{v}_{i}=E_{\theta}\left[\tilde{g}_{i}\left(\mathbf{x}_{j}^{*}, \theta\right)\right]$, where $\mathbf{x}_{j}^{*} \in \arg \max _{\mathbf{x}} E_{\theta}\left[\tilde{g}_{j}(\mathbf{x}, \theta)-f_{0}(\mathbf{x}, \theta)\right], i, j=1,2, j \neq i$, we obtain

$$
\begin{aligned}
& v(\{1\})+\underline{v}_{2} \leq v(\{1,2\}), \\
& v(\{2\})+\underline{v}_{1} \leq v(\{1,2\}),
\end{aligned}
$$

which, together with $v_{i}^{*}=\Lambda-v(\{j\}), i, j=1,2, j \neq i$, as established above, implies that $\underline{v}_{i} \leq v_{i}^{*}$.

\section{A.5 Bilateral profit maximization.}

We demonstrate that an element of

$$
X\left(\tilde{t}_{i}, t_{j}\right)=\arg \max _{\mathbf{x}}\left\{E_{\theta}\left[\tilde{t}_{i}(\mathbf{x}, \theta)+t_{j}(\mathbf{x}, \theta)-f_{0}(\mathbf{x}, \theta)\right]\right\}
$$

the set of actions that maximize the expected payoff to the agent (the lab) given the principals' (firms') strategies $\left(\tilde{t}_{i}, t_{j}\right)$, is also an element of

$$
X_{\{i\}}^{*}=\arg \max _{\mathbf{x}}\left\{E_{\theta}\left[\tilde{g}_{i}(\mathbf{x}, \theta)+t_{j}(\mathbf{x}, \theta)-f_{0}(\mathbf{x}, \theta)\right]\right\},
$$

the set of actions that maximize the joint payoff to the agent and principal $i$. That is:

Lemma A.5 If $\tilde{\mathbf{x}}$ is an element of $X\left(\tilde{t}_{i}, t_{j}\right)$, then $\tilde{\mathbf{x}}$ is also an element of $X_{\{i\}}^{*}$. 
Proof. We suppose that $\tilde{\mathbf{x}} \notin X_{\{i\}}^{*}$, and look for a contradiction. In equilibrium, the strategy $\tilde{t}_{i}$ is truthful relative to the equilibrium choice $\tilde{\mathbf{x}}$, that is $\tilde{t}_{i}(\mathbf{x}, \theta)=\sup \left\{0, \tilde{g}_{i}(\mathbf{x}, \theta)-\left[\tilde{g}_{i}(\tilde{\mathbf{x}}, \theta)-\tilde{t}_{i}(\tilde{\mathbf{x}}, \theta)\right]\right\}$, for all $\theta \in \Theta$, implying that

$$
\tilde{t}_{i}(\mathbf{x}, \theta) \geq \tilde{g}_{i}(\mathbf{x}, \theta)-\left[\tilde{g}_{i}(\tilde{\mathbf{x}}, \theta)-\tilde{t}_{i}(\tilde{\mathbf{x}}, \theta)\right]
$$

all $\theta \in \Theta$, all $\mathbf{x}$. This holds in particular for any given $\mathbf{x}^{*} \in X_{\{i\}}^{*}$, so that $\tilde{t}_{i}\left(\mathbf{x}^{*}, \theta\right) \geq \tilde{g}_{i}\left(\mathbf{x}^{*}, \theta\right)-$ $\left[\tilde{g}_{i}(\tilde{\mathbf{x}}, \theta)-\tilde{t}_{i}(\tilde{\mathbf{x}}, \theta)\right]$, all $\theta \in \Theta$. Adding $t_{j}\left(\mathbf{x}^{*}, \theta\right)-f_{0}\left(\mathbf{x}^{*}, \theta\right)$ on each side of the inequality, leads to

$$
v_{0}\left(\mathbf{x}^{*}, \theta\right) \geq \tilde{g}_{i}\left(\mathbf{x}^{*}, \theta\right)+t_{j}\left(\mathbf{x}^{*}, \theta\right)-f_{0}\left(\mathbf{x}^{*}, \theta\right)-\tilde{g}_{i}(\tilde{\mathbf{x}}, \theta)+\tilde{t}_{i}(\tilde{\mathbf{x}}, \theta) .
$$

By introducing $t_{j}(\tilde{\mathbf{x}}, \theta)-f_{0}(\tilde{\mathbf{x}}, \theta)$ on the right-hand side only, and reorganizing terms, we obtain

$$
\begin{aligned}
v_{0}\left(\mathbf{x}^{*}, \theta\right) \geq & {\left[\tilde{g}_{i}\left(\mathbf{x}^{*}, \theta\right)+t_{j}\left(\mathbf{x}^{*}, \theta\right)-f_{0}\left(\mathbf{x}^{*}, \theta\right)\right] } \\
& -\left[\tilde{g}_{i}(\tilde{\mathbf{x}}, \theta)+t_{j}(\tilde{\mathbf{x}}, \theta)-f_{0}(\tilde{\mathbf{x}}, \theta)\right]+\tilde{t}_{i}(\tilde{\mathbf{x}}, \theta)+t_{j}(\tilde{\mathbf{x}}, \theta)-f_{0}(\tilde{\mathbf{x}}, \theta),
\end{aligned}
$$

all $\theta \in \Theta$. Obviously, if this holds for all $\theta \in \Theta$, this is also true in expectation, that is

$$
\begin{aligned}
E_{\theta}\left[v_{0}\left(\mathbf{x}^{*}, \theta\right)\right] \geq & E_{\theta}\left[\tilde{g}_{i}\left(\mathbf{x}^{*}, \theta\right)+t_{j}\left(\mathbf{x}^{*}, \theta\right)-f_{0}\left(\mathbf{x}^{*}, \theta\right)\right] \\
& -E_{\theta}\left[\tilde{g}_{i}(\tilde{\mathbf{x}}, \theta)+t_{j}(\tilde{\mathbf{x}}, \theta)-f_{0}(\tilde{\mathbf{x}}, \theta)\right]+E_{\theta}\left[v_{0}(\tilde{\mathbf{x}}, \theta)\right] .
\end{aligned}
$$

Observe that $\mathbf{x}^{*} \in X_{\{i\}}^{*}$ and $\tilde{\mathbf{x}} \notin X_{\{i\}}^{*}$ together imply that $E_{\theta}\left[\tilde{g}_{i}\left(\mathbf{x}^{*}, \theta\right)+t_{j}\left(\mathbf{x}^{*}, \theta\right)-f_{0}\left(\mathbf{x}^{*}, \theta\right)\right]>$ $E_{\theta}\left[\tilde{g}_{i}(\tilde{\mathbf{x}}, \theta)+t_{j}(\tilde{\mathbf{x}}, \theta)-f_{0}(\tilde{\mathbf{x}}, \theta)\right]$, which in turn implies from (58) that

$$
E_{\theta}\left[v_{0}\left(\mathbf{x}^{*}, \theta\right)\right]>E_{\theta}\left[v_{0}(\tilde{\mathbf{x}}, \theta)\right]
$$

The latter comparison says that $\tilde{\mathbf{x}} \notin X\left(\tilde{t}_{i}, t_{j}\right)$, a contradiction. Therefore, $\tilde{\mathbf{x}} \in X_{\{i\}}^{*}$.

\section{A.6 Proof of Lemma 1.}

Consider $\mathbf{x}^{o} \in X\left(t_{i}^{o}, t_{j}\right)$, a choice of the agent (the lab) when it faces principal (firm) $i^{\prime}$ s strategy (contract offer) $t_{i}^{o}(\mathbf{x}, \theta)$, the other strategy $t_{j}(\mathbf{x}, \theta)$ remaining unchanged. Formally,

$$
\mathbf{x}^{o} \in \arg \max _{\mathbf{x} \in X}\left\{E_{\theta}\left[t_{i}^{o}(\mathbf{x}, \theta)+t_{j}(\mathbf{x}, \theta)-f_{0}(\mathbf{x}, \theta)\right]\right\}
$$

We want to prove that $\mathbf{x}^{o}$ cannot be distinguished from the action $\tilde{\mathbf{x}}$, as chosen by the agent when principal $i$ 's strategy is $\tilde{t}_{i}(\mathbf{x}, \theta)$. That is, we want to establish that

$$
\mathbf{x}^{o} \in \arg \max _{\mathbf{x} \in X}\left\{E_{\theta}\left[\tilde{t}_{i}(\mathbf{x}, \theta)+t_{j}(\mathbf{x}, \theta)-f_{0}(\mathbf{x}, \theta)\right]\right\},
$$


and that the strategy $t_{i}^{o}$ results in the same expected payoff to the agent and both principals.

(i) We first prove that $\tilde{\mathbf{x}}$ leads to the same expected payoff to the agent when facing $t_{i}^{o}(\mathbf{x}, \theta)$ as when facing $\tilde{t}_{i}(\mathbf{x}, \theta)$ so that principal $i^{\prime}$ s change of strategy from $\tilde{t}_{i}$ to $t_{i}^{o}$ should not result in the agent turning down the new contract offer. Toward this aim, observe that $v_{i}(\tilde{\mathbf{x}}, \tilde{\mathbf{y}}, \tilde{\mathbf{z}}, \theta)=\tilde{g}_{i}(\tilde{\mathbf{x}}, \theta)-\tilde{t}_{i}(\tilde{\mathbf{x}}, \theta)$, by definition, for all $\theta \in \Theta$. From inequality (21), we have

$$
h(\theta)+\tilde{t}_{i}(\tilde{\mathbf{x}}, \theta) \geq \tilde{g}_{i}(\tilde{\mathbf{x}}, \theta)-\underline{g}(\theta) \geq 0,
$$

so that

$$
v_{i}(\tilde{\mathbf{x}}, \tilde{\mathbf{y}}, \tilde{\mathbf{z}}, \theta)-h(\theta)=\tilde{g}_{i}(\tilde{\mathbf{x}}, \theta)-\left[\tilde{t}_{i}(\tilde{\mathbf{x}}, \theta)+h(\theta)\right] \leq \tilde{g}_{i}(\tilde{\mathbf{x}}, \theta),
$$

for all $\theta \in \Theta$. As a result

$$
t_{i}^{o}(\tilde{\mathbf{x}}, \theta)=\sup \left\{0, \tilde{g}_{i}(\tilde{\mathbf{x}}, \theta)-\left[v_{i}(\tilde{\mathbf{x}}, \tilde{\mathbf{y}}, \tilde{\mathbf{z}}, \theta)-h(\theta)\right]\right\}=\tilde{g}_{i}(\tilde{\mathbf{x}}, \theta)-\left[v_{i}(\tilde{\mathbf{x}}, \tilde{\mathbf{y}}, \tilde{\mathbf{z}}, \theta)-h(\theta)\right]
$$

for all $\theta \in \Theta$, and hence

$$
\begin{aligned}
E_{\theta}\left[t_{i}^{o}(\tilde{\mathbf{x}}, \theta)+t_{j}(\tilde{\mathbf{x}}, \theta)-f_{0}(\tilde{\mathbf{x}}, \theta)\right] & =E_{\theta}\left[\tilde{g}_{i}(\tilde{\mathbf{x}}, \theta)-\left[v_{i}(\tilde{\mathbf{x}}, \tilde{\mathbf{y}}, \tilde{\mathbf{z}}, \theta)-h(\theta)\right]+t_{j}(\tilde{\mathbf{x}}, \theta)-f_{0}(\tilde{\mathbf{x}}, \theta)\right] \\
& =E_{\theta}\left[\tilde{g}_{i}(\tilde{\mathbf{x}}, \theta)-v_{i}(\tilde{\mathbf{x}}, \tilde{\mathbf{y}}, \tilde{\mathbf{z}}, \theta)+t_{j}(\tilde{\mathbf{x}}, \theta)-f_{0}(\tilde{\mathbf{x}}, \theta)\right] \\
& =E_{\theta}\left[\tilde{t}_{i}(\tilde{\mathbf{x}}, \theta)+t_{j}(\tilde{\mathbf{x}}, \theta)-f_{0}(\tilde{\mathbf{x}}, \theta)\right]
\end{aligned}
$$

where the passage from the first to the second equality is justified by the zero expected value of a lump-sum transfer adjustment (equation (20)), and the passage from the second to the third directly follows from the definition of principal $i$ 's equilibrium payoff: $v_{i}(\tilde{\mathbf{x}}, \tilde{\mathbf{y}}, \tilde{\mathbf{z}}, \theta)=\tilde{g}_{i}(\tilde{\mathbf{x}}, \theta)-\tilde{t}_{i}(\tilde{\mathbf{x}}, \theta)$.

(ii) We now prove that $\mathbf{x}^{o}$, as chosen by the agent when facing $t_{i}^{o}$, must be an element of

$$
\arg \max _{\mathbf{x} \in X}\left\{E_{\theta}\left[\tilde{t}_{i}(\mathbf{x}, \theta)+t_{j}(\mathbf{x}, \theta)-f_{0}(\mathbf{x}, \theta)\right]\right\}
$$

so that, for principal $i$, it is indistinguishable from the action $\tilde{\mathbf{x}}$ chosen when the agent faces $\tilde{t}_{i}$. To see that, recall that by construction $t_{i}^{o}(\mathbf{x}, \theta)=\sup \left\{0, \tilde{g}_{i}(\mathbf{x}, \theta)-\left[v_{i}(\tilde{\mathbf{x}}, \tilde{\mathbf{y}}, \tilde{\mathbf{z}}, \theta)-h(\theta)\right]\right\}$ is truthful with respect to $\tilde{\mathbf{x}}$ as associated to the equilibrium payoff $v_{i}(\tilde{\mathbf{x}}, \tilde{\mathbf{y}}, \tilde{\mathbf{z}}, \theta)-h(\theta) \geq 0$. Then, as established by Lemma (A.5), truthful equilibria are (bilaterally) efficient so that it must be the case that

$$
E_{\theta}\left[\tilde{g}_{i}\left(\mathbf{x}^{o}, \theta\right)+t_{j}\left(\mathbf{x}^{o}, \theta\right)-f_{0}\left(\mathbf{x}^{o}, \theta\right)\right]=E_{\theta}\left[\tilde{g}_{i}(\tilde{\mathbf{x}}, \theta)+t_{j}(\tilde{\mathbf{x}}, \theta)-f_{0}(\tilde{\mathbf{x}}, \theta)\right] .
$$


It follows that

$$
\begin{aligned}
E_{\theta}\left[\tilde{t}_{i}\left(\mathbf{x}^{o}, \theta\right)+t_{j}\left(\mathbf{x}^{o}, \theta\right)-f_{0}\left(\mathbf{x}^{o}, \theta\right)\right] & \geq E_{\theta}\left[\tilde{g}_{i}\left(\mathbf{x}^{o}, \theta\right)-v_{i}(\tilde{\mathbf{x}}, \tilde{\mathbf{y}}, \tilde{\mathbf{z}}, \theta)+t_{j}\left(\mathbf{x}^{o}, \theta\right)-f_{0}\left(\mathbf{x}^{o}, \theta\right)\right] \\
& =E_{\theta}\left[\tilde{g}_{i}\left(\mathbf{x}^{o}, \theta\right)+t_{j}\left(\mathbf{x}^{o}, \theta\right)-f_{0}\left(\mathbf{x}^{o}, \theta\right)\right]-E_{\theta}\left[v_{i}(\tilde{\mathbf{x}}, \tilde{\mathbf{y}}, \tilde{\mathbf{z}}, \theta)\right] \\
& =E_{\theta}\left[\tilde{g}_{i}(\tilde{\mathbf{x}}, \theta)+t_{j}(\tilde{\mathbf{x}}, \theta)-f_{0}(\tilde{\mathbf{x}}, \theta)\right]-E_{\theta}\left[v_{i}(\tilde{\mathbf{x}}, \tilde{\mathbf{y}}, \tilde{\mathbf{z}}, \theta)\right] \\
& =E_{\theta}\left[\tilde{g}_{i}(\tilde{\mathbf{x}}, \theta)-v_{i}(\tilde{\mathbf{x}}, \tilde{\mathbf{y}}, \tilde{\mathbf{z}}, \theta)+t_{j}(\tilde{\mathbf{x}}, \theta)-f_{0}(\tilde{\mathbf{x}}, \theta)\right] \\
& =E_{\theta}\left[\tilde{t}_{i}(\tilde{\mathbf{x}}, \theta)+t_{j}(\tilde{\mathbf{x}}, \theta)-f_{0}(\tilde{\mathbf{x}}, \theta)\right],
\end{aligned}
$$

where the inequality follows from the definition of the truthful strategy $\tilde{t}_{i}$, the equality with the second line follows from the linearity of the expectation operator, the equality with the third line from equation (61), the equality with the fourth line uses again the linearity of the expectation operator, and finally the bottom line follows from the definition of principal $i$ 's equilibrium payoff $v_{i}(\tilde{\mathbf{x}}, \tilde{\mathbf{y}}, \tilde{\mathbf{z}}, \theta)$.Thus, the agent's expected profit, when offered $\tilde{t}_{i}$ by principal $i$ and choosing $\mathbf{x}^{o}$, is at least as high as the one earned by choosing $\tilde{\mathbf{x}}$. Because the latter action maximizes the agent's payoff when offered $\tilde{t}_{i}$ by principal $i$, the inequality cannot be strict. Therefore,

$$
E_{\theta}\left[\tilde{t}_{i}\left(\mathbf{x}^{o}, \theta\right)+t_{j}\left(\mathbf{x}^{o}, \theta\right)-f_{0}\left(\mathbf{x}^{o}, \theta\right)\right]=E_{\theta}\left[\tilde{t}_{i}(\tilde{\mathbf{x}}, \theta)+t_{j}(\tilde{\mathbf{x}}, \theta)-f_{0}(\tilde{\mathbf{x}}, \theta)\right] .
$$

(iii) We now prove that, by offering $t_{i}^{o}$ rather than $\tilde{t}_{i}$, principal $i$ is not conceding more profit to the agent. Indeed, by definition, and using the linearity of the expectation operator again, we have

$$
\begin{aligned}
E_{\theta}\left[t_{i}^{o}(\tilde{\mathbf{x}}, \theta)+t_{j}(\tilde{\mathbf{x}}, \theta)-f_{0}(\tilde{\mathbf{x}}, \theta)\right] & \geq E_{\theta}\left[\tilde{g}_{i}(\tilde{\mathbf{x}}, \theta)-\left[v_{i}(\tilde{\mathbf{x}}, \tilde{\mathbf{y}}, \tilde{\mathbf{z}}, \theta)-h(\theta)\right]+t_{j}(\tilde{\mathbf{x}}, \theta)-f_{0}(\tilde{\mathbf{x}}, \theta)\right] \\
& =E_{\theta}\left[\tilde{g}_{i}(\tilde{\mathbf{x}}, \theta)+t_{j}(\tilde{\mathbf{x}}, \theta)-f_{0}(\tilde{\mathbf{x}}, \theta)\right]-E_{\theta}\left[v_{i}(\tilde{\mathbf{x}}, \tilde{\mathbf{y}}, \tilde{\mathbf{z}}, \theta)-h(\theta)\right] \\
& =E_{\theta}\left[\tilde{g}_{i}\left(\mathbf{x}^{o}, \theta\right)+t_{j}\left(\mathbf{x}^{o}, \theta\right)-f_{0}\left(\mathbf{x}^{o}, \theta\right)\right]-E_{\theta}\left[v_{i}(\tilde{\mathbf{x}}, \tilde{\mathbf{y}}, \tilde{\mathbf{z}}, \theta)-h(\theta)\right] \\
& =E_{\theta}\left[\tilde{g}_{i}\left(\mathbf{x}^{o}, \theta\right)-\left[v_{i}(\tilde{\mathbf{x}}, \tilde{\mathbf{y}}, \tilde{\mathbf{z}}, \theta)-h(\theta)\right]+t_{j}\left(\mathbf{x}^{o}, \theta\right)-f_{0}\left(\mathbf{x}^{o}, \theta\right)\right],
\end{aligned}
$$

where the inequality follows from the definition of the truthful strategy $t_{i}^{o}$, and the equality between the second and third lines follows from equation (61). We also know that $\tilde{g}_{i}\left(\mathbf{x}^{o}, \theta\right) \geq g(\theta)$ by definition (lower bound), and that $\underline{g}(\theta) \geq v_{i}(\tilde{\mathbf{x}}, \tilde{\mathbf{y}}, \tilde{\mathbf{z}}, \theta)-h(\theta)$ from the first inequality in $(21)$, so

$$
\tilde{g}_{i}\left(\mathbf{x}^{o}, \theta\right) \geq \underline{g}(\theta) \geq v_{i}(\tilde{\mathbf{x}}, \tilde{\mathbf{y}}, \tilde{\mathbf{z}}, \theta)-h(\theta),
$$

and hence

$$
\begin{aligned}
t_{i}^{o}\left(\mathbf{x}^{o}, \theta\right) & =\sup \left\{0, \tilde{g}_{i}\left(\mathbf{x}^{o}, \theta\right)-\left[v_{i}(\tilde{\mathbf{x}}, \tilde{\mathbf{y}}, \tilde{\mathbf{z}}, \theta)-h(\theta)\right]\right\} \\
& =\tilde{g}_{i}\left(\mathbf{x}^{o}, \theta\right)-\left[v_{i}(\tilde{\mathbf{x}}, \tilde{\mathbf{y}}, \tilde{\mathbf{z}}, \theta)-h(\theta)\right]
\end{aligned}
$$


for all $\theta \in \Theta$, then we have

$$
\begin{aligned}
E_{\theta}\left[t_{i}^{o}(\tilde{\mathbf{x}}, \theta)+t_{j}(\tilde{\mathbf{x}}, \theta)-f_{0}(\tilde{\mathbf{x}}, \theta)\right] & \geq E_{\theta}\left[t_{i}^{o}\left(\mathbf{x}^{o}, \theta\right)+t_{j}\left(\mathbf{x}^{o}, \theta\right)-f_{0}\left(\mathbf{x}^{o}, \theta\right)\right] \\
& =\max _{\mathbf{x} \in X} E_{\theta}\left[t_{i}^{o}(\mathbf{x}, \theta)+t_{j}(\mathbf{x}, \theta)-f_{0}(\mathbf{x}, \theta)\right],
\end{aligned}
$$

which, by definition of a maximum, cannot be strict. Thus, there is no other efficient choice $\mathbf{x}^{o}$ that results in a higher payoff than $\tilde{\mathbf{x}}$ when the agent is offered $t_{i}^{o}$ by principal $i$.

As we have already shown that the choice of $\tilde{\mathbf{x}}$ implies the same payoff to the agent whether offered $t_{i}^{o}$ or $\tilde{t}_{i}$ (see equation (60)), the agent cannot improve on its expected payoff when facing the first strategy rather than the second.

(iv) From Lemma (A.5), because both strategies are truthful their associated choices are efficient, that is the expected joint profit of principal $i$ and the agent is a maximum and hence unchanged whether the agent is offered $t_{i}^{o}(\mathbf{x}, \theta)$ or $\tilde{t}_{i}(\mathbf{x}, \theta)$. Since we have just shown that the expected payoff of the agent is also unchanged, the one of principal $i$ must also be unchanged.

Eventually, from the previous steps we obtain that, when offering the strategy $t_{i}^{o}$ rather than $\tilde{t}_{i}$, principal $i$ still induces the agent to make efficient choices, and the expected payoffs to the agent and both principals end up unchanged. However, from equation (63) we obtain that principal $i$ 's realized (ex-post) payoff is now given by $v_{i}(\tilde{\mathbf{x}}, \tilde{\mathbf{y}}, \tilde{\mathbf{z}}, \theta)-h(\theta)$, whereas the one of the agent is now equal to $v_{0}(\tilde{\mathbf{x}}, \theta)+h(\theta)$.

\section{A.7 Proof of Proposition 4.}

(i) Suppose first that $\underline{g}(\theta) \leq E_{\theta}\left[\tilde{g}_{i}(\tilde{\mathbf{x}}, \theta)-\tilde{t}_{i}(\tilde{\mathbf{x}}, \theta)\right]$ for a subset of $\Theta$ with positive measure.

We define the set $\underline{\Theta}(\bar{v})$ such that $\underline{g}(\theta) \leq \bar{v}$, with $\bar{v} \geq v_{i}^{*}$ (so that it is a non-empty set), and the function $h: \Theta \rightarrow \mathcal{R}$ such that:

$$
h(\theta)= \begin{cases}-\left[g(\theta)-v_{i}(\tilde{\mathbf{x}}, \tilde{\mathbf{y}}, \tilde{\mathbf{z}}, \theta)\right] & \text { for all } \theta \in \underline{\Theta}(\bar{v}), \\ v_{i}(\tilde{\mathbf{x}}, \tilde{\mathbf{y}}, \tilde{\mathbf{z}}, \theta)-\bar{v} & \text { otherwise. }\end{cases}
$$

Then, the truthful strategy $t_{i}^{o}(\mathbf{x}, \theta)=\sup \left\{0, \tilde{t}_{i}(\mathbf{x}, \theta)+h(\theta)\right\}$ is such that

$$
t_{i}^{o}(\mathbf{x}, \theta)= \begin{cases}\sup \left\{0, \tilde{g}_{i}(\mathbf{x}, \theta)-\underline{g}(\theta)\right\} & \text { for all } \theta \in \underline{\Theta}(\overline{\mathcal{v}}), \\ \sup \left\{0, \tilde{g}_{i}(\mathbf{x}, \theta)-\bar{v}\right\} & \text { otherwise. }\end{cases}
$$

In equilibrium, the principal $i$ realized payoff $v_{i}(\tilde{\mathbf{x}}, \tilde{\mathbf{y}}, \tilde{\mathbf{z}}, \theta)-h(\theta)$ are

$$
v_{i}(\tilde{\mathbf{x}}, \tilde{\mathbf{y}}, \tilde{\mathbf{z}}, \theta)-h(\theta)= \begin{cases}g(\theta) & \text { for all } \theta \in \underline{\Theta}(\overline{\mathcal{v}}) \\ \overline{\bar{v}} & \text { otherwise. }\end{cases}
$$


(ii) Suppose now that $\underline{g}(\theta)>E_{\theta}\left[\tilde{g}_{i}(\tilde{\mathbf{x}}, \theta)-\tilde{t}_{i}(\tilde{\mathbf{x}}, \theta)\right]$ for almost all $\theta \in \Theta$.

Let $h(\theta)=v_{i}(\tilde{\mathbf{x}}, \tilde{\mathbf{y}}, \tilde{\mathbf{z}}, \theta)-v_{i}^{*}$, with $v_{i}^{*}=E_{\theta}\left[v_{i}(\tilde{\mathbf{x}}, \tilde{\mathbf{y}}, \tilde{\mathbf{z}}, \theta)\right]$, so that $E_{\theta}[h(\theta)]=0$, and

$$
\tilde{g}_{i}(\tilde{\mathbf{x}}, \theta)-\underline{g}(\theta)-\tilde{t}_{i}(\tilde{\mathbf{x}}, \theta)<\tilde{g}_{i}(\tilde{\mathbf{x}}, \theta)-\tilde{t}_{i}(\tilde{\mathbf{x}}, \theta)-v_{i}^{*} \leq \tilde{g}_{i}(\tilde{\mathbf{x}}, \theta)-\tilde{t}_{i}(\tilde{\mathbf{x}}, \theta) .
$$

Then, from Lemma 1, the truthful strategy

$$
t_{i}^{o}(\mathbf{x}, \theta)=\sup \left\{0, \tilde{g}_{i}(\mathbf{x}, \theta)-\left[v_{i}(\tilde{\mathbf{x}}, \tilde{\mathbf{y}}, \tilde{\mathbf{z}}, \theta)-h(\theta)\right]\right\}=\sup \left\{0, \tilde{g}_{i}(\mathbf{x}, \theta)-v_{i}^{*}\right\}
$$

is a best-response strategy to $\tilde{t}_{j}$, for the same equilibrium outcomes $(\tilde{\mathbf{x}}, \tilde{\mathbf{y}}, \tilde{\mathbf{z}})$ and a realized equilibrium payoff $v_{i}(\tilde{\mathbf{x}}, \tilde{\mathbf{y}}, \tilde{\mathbf{z}}, \theta)-h(\theta)=v_{i}^{*}$ which is independent of $\theta$.

\section{A.8 Proof of Proposition 5.}

First, Lemma A.1 extends to the case $\frac{\partial^{2} \hat{g}_{i}}{\partial x_{i}^{2}} \geq 0(i=1,2)$. Indeed we have established in (48) that $\frac{\partial^{2} \hat{g}_{i}}{\partial x_{i}^{2}}=0 \Rightarrow \frac{d y_{j}^{*}}{d x_{j}}=0$, and in (51) that $\frac{\partial^{2} \hat{g}_{i}}{\partial x_{i}^{2}}>0 \Rightarrow \frac{d y_{j}^{*}}{d x_{j}}>0$. This is sufficient to conclude directly that $\frac{d\left[\hat{g}_{i}\left(x_{i}+y_{i}^{*}(\mathbf{x}), x_{j}, y_{j}^{*}(\mathbf{x})\right)-\hat{f}_{i}\left(y_{i}^{*}(\mathbf{x})\right)\right]}{d x_{j}}$ in (52) has the same sign as $\frac{\partial \hat{g}_{i}\left(x_{i}+y_{i}, x_{j}, y_{j}\right)}{\partial x_{j}}$ and $\frac{\partial \hat{g}_{i}\left(x_{i}+y_{i}, x_{j}, y_{j}\right)}{\partial y_{j}}$.

To extend Lemma A.2 as well, note that $\frac{\partial^{2} \hat{g}_{i}}{\partial x_{i}^{2}} \geq 0(i=1,2)$ implies $\frac{N_{j i}}{\Delta} \geq 0$ in (55), because $\Delta>0$ (from stability condition) and $N_{j i} \geq 0$ from $\frac{\partial^{2} \hat{g}_{j}}{\partial x_{j}^{2}}-\frac{\partial^{2} \hat{f}_{j}}{\partial y_{j}^{2}}<0$ (second-order condition) and $\frac{\partial^{2} \hat{g}_{j}}{\partial x_{j}^{2}} \geq 0$ (assumption in this proposition). Then there are only two possible cases:

(i) If $\frac{\partial^{2} \hat{g}_{j}}{\partial x_{j} \partial x_{i}} \geq 0$ then $\frac{\partial^{2} \hat{g}_{j}}{\partial x_{j} \partial y_{i}} \geq 0$ also (model specifications). As $\frac{N_{j i}}{\Delta} \geq 0$, we obtain that the expression between brackets in (55) is positive. Moreover, $\frac{\partial^{2} \hat{g}_{j}}{\partial x_{j}^{2}}-\frac{\partial^{2} \hat{f}_{j}}{\partial y_{j}^{2}}<0$ (second-order condition) here implies that $-\frac{\partial^{2} \hat{g}_{j}}{\partial x_{j} \partial x_{i}}\left(\frac{\partial^{2} \hat{g}_{j}}{\partial x_{j}^{2}}-\frac{\partial^{2} \hat{f}_{j}}{\partial y_{j}^{2}}\right)^{-1} \geq 0$. Therefore, from (55) we have $\frac{d y_{j}^{*}}{d x_{i}} \geq 0$, which is sufficient to conclude directly that $\frac{d\left[\hat{g}_{i}\left(x_{i}+y_{i}^{*}(\mathbf{x}), x_{j}, y_{j}^{*}(\mathbf{x})\right)-\hat{f}_{i}\left(y_{i}^{*}(\mathbf{x})\right)\right]}{d x_{i}}$ in (54) is non-negative also.

(ii) If $\frac{\partial^{2} \hat{g}_{j}}{\partial x_{j} \partial x_{i}} \leq 0$ then $\frac{\partial^{2} \hat{g}_{j}}{\partial x_{j} \partial y_{i}} \leq 0$ also (model specifications). As $\frac{N_{j i}}{\Delta} \geq 0$, again the expression between brackets in (55) is positive. Moreover, $\frac{\partial^{2} \hat{g}_{j}}{\partial x_{j}^{2}}-\frac{\partial^{2} \hat{f}_{j}}{\partial y_{j}^{2}}<0$ (second-order condition) implies here that $-\frac{\partial^{2} \hat{\mathrm{g}}_{j}}{\partial x_{j} \partial x_{i}}\left(\frac{\partial^{2} \hat{\mathrm{g}}_{j}}{\partial x_{j}^{2}}-\frac{\partial^{2} \hat{f}_{j}}{\partial y_{j}^{2}}\right)^{-1} \leq 0$. Therefore, from (55) we have that $\frac{d y_{j}^{*}}{d x_{i}} \leq 0$, implying that $\left\|\frac{d y_{j}^{*}}{d x_{i}}\right\|=-\frac{d y_{j}^{*}}{d x_{i}}$. So, recalling that $\left\|\frac{\partial \hat{g}_{i}}{\partial x_{j}}\right\| \geq\left\|\frac{\partial \hat{g}_{i}}{\partial y_{j}}\right\|$ (model specifications in (6-7)), a sufficient condition for Lemma A.2 to be robust to the increasing $\mathrm{R} \& \mathrm{D}$ return specification is $\frac{d y_{j}^{*}}{d x_{i}}>-1$. 


\section{A.9 Proof of industry profit maximization result in Proposition 6.}

Recall from the model specifications in Section 3 that, in equilibrium, for any given pair of transfer payment functions $\left(\tilde{t}_{1}, \tilde{t}_{2}\right)$ we know that $\tilde{\mathbf{x}}$ is an element of $X\left(\tilde{t}_{1}, \tilde{t}_{2}\right)=\arg \max _{\mathbf{x}}\left\{E_{\theta}\left[v_{0}\left(\mathbf{x}\left(\tilde{t}_{1}, \tilde{t}_{2}\right), \theta\right)\right]\right\}$, the set of external R\&D choices that maximize the lab's profit. We want to demonstrate that $\tilde{\mathbf{x}}$ is also an element of $X_{\{1,2\}}^{*}=\arg \max _{\mathbf{x}}\left\{E_{\theta}\left[\tilde{g}_{1}(\mathbf{x}, \theta)+\tilde{g}_{2}(\mathbf{x}, \theta)-f_{0}(\mathbf{x}, \theta)\right]\right\}$, the set of external R\&D levels that maximize industry profit. The proof is a simple adaptation, in the notation of our model, of a common agency efficiency result in Bernheim and Whinston (1986b, Theorem 2, p. 14).

We suppose that $\tilde{\mathbf{x}} \notin X_{\{1,2\}}^{*}$, and look for a contradiction. In equilibrium, the strategy $\tilde{t}_{i}$ is truthful relative to the equilibrium choice $\tilde{\mathbf{x}}$ for all $\theta \in \Theta$, that is $\tilde{t}_{i}(\mathbf{x}, \theta)=\sup \left\{0, \tilde{g}_{i}(\mathbf{x}, \theta)-\left[\tilde{g}_{i}(\tilde{\mathbf{x}}, \theta)-\tilde{t}_{i}(\tilde{\mathbf{x}}, \theta)\right]\right\}$, all $\theta \in \Theta$, all $\mathbf{x}$, implying that

$$
\tilde{g}_{i}(\mathbf{x}, \theta)-\left[\tilde{g}_{i}(\tilde{\mathbf{x}}, \theta)-\tilde{t}_{i}(\tilde{\mathbf{x}}, \theta)\right] \leq \tilde{t}_{i}(\mathbf{x}, \theta)
$$

This holds in particular for any given $\mathbf{x}^{*} \in X_{\{1,2\}}^{*}$, so that $\tilde{g}_{i}\left(\mathbf{x}^{*}, \theta\right)-\left[\tilde{g}_{i}(\tilde{\mathbf{x}}, \theta)-\tilde{t}_{i}(\tilde{\mathbf{x}}, \theta)\right] \leq \tilde{t}_{i}\left(\mathbf{x}^{*}, \theta\right)$, all $\theta \in \Theta, i=1$, 2. Summing the latter inequality for the two firms, and subtracting $f_{0}\left(\mathbf{x}^{*}, \theta\right)$ on each side, leads to

$$
\tilde{g}\left(\mathbf{x}^{*}, \theta\right)-\tilde{g}(\tilde{\mathbf{x}}, \theta)+\tilde{t}(\tilde{\mathbf{x}}, \theta)-f_{0}\left(\mathbf{x}^{*}, \theta\right) \leq \tilde{t}\left(\mathbf{x}^{*}, \theta\right)-f_{0}\left(\mathbf{x}^{*}, \theta\right),
$$

all $\theta \in \Theta$, where $\tilde{g}(\mathbf{x}, \theta)=\tilde{g}_{1}(\mathbf{x}, \theta)+\tilde{g}_{2}(\mathbf{x}, \theta)$, and $\tilde{t}(\mathbf{x}, \theta)=\tilde{t}_{1}(\mathbf{x}, \theta)+\tilde{t}_{2}(\mathbf{x}, \theta)$. By introducing $f_{0}(\tilde{\mathbf{x}}, \theta)$ on the left-hand side only, and reorganizing terms, we obtain

$$
\left[\tilde{g}\left(\mathbf{x}^{*}, \theta\right)-f_{0}\left(\mathbf{x}^{*}, \theta\right)\right]-\left[\tilde{g}(\tilde{\mathbf{x}}, \theta)-f_{0}(\tilde{\mathbf{x}}, \theta)\right]+\tilde{t}(\tilde{\mathbf{x}}, \theta)-f_{0}(\tilde{\mathbf{x}}, \theta) \leq \tilde{t}\left(\mathbf{x}^{*}, \theta\right)-f_{0}\left(\mathbf{x}^{*}, \theta\right),
$$

all $\theta \in \Theta$. Obviously, if this inequality holds for all $\theta \in \Theta$, it holds also in expectation, that is

$$
E_{\theta}\left[\tilde{g}\left(\mathbf{x}^{*}, \theta\right)-f_{0}\left(\mathbf{x}^{*}, \theta\right)\right]-E_{\theta}\left[\tilde{g}(\tilde{\mathbf{x}}, \theta)-f_{0}(\tilde{\mathbf{x}}, \theta)\right]+E_{\theta}\left[\tilde{t}(\tilde{\mathbf{x}}, \theta)-f_{0}(\tilde{\mathbf{x}}, \theta)\right] \leq E_{\theta}\left[\tilde{t}\left(\mathbf{x}^{*}, \theta\right)-f_{0}\left(\mathbf{x}^{*}, \theta\right)\right]
$$

Observe that $\mathbf{x}^{*} \in X_{\{1,2\}}^{*}$ and $\tilde{\mathbf{x}} \notin X_{\{1,2\}}^{*}$ together imply that $E_{\theta}\left[\tilde{g}\left(\mathbf{x}^{*}, \theta\right)-f_{0}\left(\mathbf{x}^{*}, \theta\right)\right]>E_{\theta}\left[\tilde{g}(\tilde{\mathbf{x}}, \theta)-f_{0}(\tilde{\mathbf{x}}, \theta)\right]$, which in turn implies from (64) that

$$
E_{\theta}\left[v_{0}(\tilde{\mathbf{x}}, \theta)\right]=E_{\theta}\left[\tilde{t}(\tilde{\mathbf{x}}, \theta)-f_{0}(\tilde{\mathbf{x}}, \theta)\right]<E_{\theta}\left[\tilde{t}\left(\mathbf{x}^{*}, \theta\right)-f_{0}\left(\mathbf{x}^{*}, \theta\right)\right]=E_{\theta}\left[v_{0}\left(\mathbf{x}^{*}, \theta\right)\right] .
$$

The latter comparison says that $\tilde{\mathbf{x}} \notin X\left(\tilde{t}_{1}, \tilde{t}_{2}\right)$, a contradiction. Therefore, $\tilde{\mathbf{x}} \in X_{\{1,2\}}^{*}$.

\section{A.10 Proof of $v_{0}^{\{0,1\}}=v_{0}^{\{0,2\}} \geq v_{0}^{*}=|\epsilon|$ in Proposition 8 (for $\epsilon<0$ ).}

Suppose that $\epsilon<0$, and consider firm $i$ 's two alternatives: if it acquires the lab, as an integrated entity it earns $\Lambda-v_{j}^{\{0, i\}}$; otherwise, as an outsider it earns $v_{i}^{\{0, j\}}$. The difference of the latter two payoffs is 
firm $i$ 's willingness to pay for the lab, which is equal to the one of firm $j$. Therefore, competition for the acquisition of the lab implies that in equilibrium $v_{0}^{\{0, i\}}=v_{0}^{\{0, j\}}=\Lambda-v_{1}^{\{0,2\}}-v_{2}^{\{0,1\}}$.

Suppose now that firm $i$ is the one that acquires the lab, while firm $j$ remains independent, $i, j=$ $1,2, j \neq i$. In the latter industry structure, the integrated entity $\{0, i\}$ and firm $j$ bargain over the value generated by the acquired lab, with respective disagreement payoffs $v(\{i\})$ and $\underline{v}_{j}$. Firm $j^{\prime}$ s payoff is thus

$$
v_{j}^{\{0, i\}}=\underline{v}_{j}+w_{j}\left(\Lambda-\underline{v}_{j}-v(\{i\})\right),
$$

where from (30) firm $j$ 's bargaining power is

$$
w_{j}=\frac{v_{j}^{\{1,2\}}-v_{j}^{*}}{\Lambda-v_{1}^{*}-v_{2}^{*}} .
$$

Given that $v_{0}^{\{0, i\}}=\Lambda-v_{1}^{\{0,2\}}-v_{2}^{\{0,1\}}$, as established above, and using (65-66), we have

$$
v_{0}^{\{0,1\}}=\Lambda-\left(\underline{v}_{1}+\frac{v_{1}^{\{1,2\}}-v_{1}^{*}}{\Lambda-v_{1}^{*}-v_{2}^{*}}\left(\Lambda-\underline{v}_{1}-v(\{2\})\right)\right)-\left(\underline{v}_{2}+\frac{v_{2}^{\{1,2\}}-v_{2}^{*}}{\Lambda-v_{1}^{*}-v_{2}^{*}}\left(\Lambda-\underline{v}_{2}-v(\{1\})\right)\right),
$$

which, by reorganizing terms, can be rewritten as

$$
v_{0}^{\{0,1\}}=\left(\frac{v(\{1\})-\underline{v}_{1}}{\Lambda-v_{1}^{*}-v_{2}^{*}}\right)\left(v_{2}^{\{1,2\}}-v_{2}^{*}\right)+\left(\frac{v(\{2\})-\underline{v}_{2}}{\Lambda-v_{1}^{*}-v_{2}^{*}}\right)\left(v_{1}^{\{1,2\}}-v_{1}^{*}\right) .
$$

Then, recalling that $v_{0}^{*}=|\epsilon|=v(\{1\})+v(\{2\})-\Lambda$, and that $\Lambda=v_{1}^{\{1,2\}}+v_{2}^{\{1,2\}}$, after a few steps we obtain that $v_{0}^{\{0,1\}} \geq v_{0}^{*}$ if and only if

$$
\left(\frac{v(\{1\})-v_{1}^{\{1,2\}}}{|\epsilon|}\right)\left[\underline{v}_{1}+v(\{2\})-\Lambda\right]+\left(\frac{v(\{2\})-v_{2}^{\{1,2\}}}{|\epsilon|}\right)\left[\underline{v}_{2}+v(\{1\})-\Lambda\right] \leq 0 .
$$

As the two added terms in (67) are symmetric, we focus on the first one:

(i) Consider the expression between square brackets. By definition of $\Lambda=\max _{\mathbf{x}} E_{\theta}\left[\tilde{g}_{1}(\mathbf{x}, \theta)+\tilde{g}_{2}(\mathbf{x}, \theta)-\right.$ $\left.f_{0}(\mathbf{x}, \theta)\right]$, we have $\Lambda \geq E_{\theta}\left[\tilde{g}_{1}\left(\mathbf{x}_{2}^{*}, \theta\right)+\tilde{g}_{2}\left(\mathbf{x}_{2}^{*}, \theta\right)-f_{0}\left(\mathbf{x}_{2}^{*}, \theta\right)\right]$, where $\mathbf{x}_{2}^{*} \in \arg \max _{\mathbf{x}} E_{\theta}\left[\tilde{g}_{2}(\mathbf{x}, \theta)-f_{0}(\mathbf{x}, \theta)\right]$. Since $\underline{v}_{1}=E_{\theta}\left[\tilde{g}_{1}\left(\mathbf{x}_{2}^{*}, \theta\right)\right]$ and $v(\{2\})=E_{\theta}\left[\tilde{g}_{2}\left(\mathbf{x}_{2}^{*}, \theta\right)-f_{0}\left(\mathbf{x}_{2}^{*}, \theta\right)\right]$, we have $\underline{v}_{1}+v(\{2\})-\Lambda \leq 0$.

(ii) Consider the numerator in the term between parentheses. From Proposition 3 we know that $v_{2}^{*}=\Lambda-v(\{1\})$. Moreover, $\Lambda-v_{1}^{*}-v_{2}^{*}=v_{0}^{*}>0$ implies from (29) that $v_{2}^{\{1,2\}}>v_{2}^{*}$ for all $\left(\omega_{1}, \omega_{2}\right)$ in $(0,1)^{2}$. It follows that $v_{2}^{\{1,2\}}>\Lambda-v(\{1\})$, and it is sufficient to recall that $v_{2}^{\{1,2\}}=\Lambda-v_{1}^{\{1,2\}}$ (the lab makes no profit in the horizontal arrangement) to establish that $v(\{1\})-v_{1}^{\{1,2\}}>0$.

Therefore, (67) is always true, with a strict inequality sign whenever $\Lambda>E_{\theta}\left[\tilde{g}_{i}\left(\mathbf{x}_{i}^{*}, \theta\right)+\tilde{g}_{j}\left(\mathbf{x}_{i}^{*}, \theta\right)-\right.$ $\left.f_{0}\left(\mathbf{x}_{i}^{*}, \theta\right)\right]$, for some $i=1,2, j \neq i$. 